\title{
Selective Oxidation of Belt[4]arene[4]tropilidene and Its Application to Construct Hydrocarbon Belts of Truncated Cone Structure with Expand Cavity
}

Yan-E Zhang, Shuo Tong, and Mei-Xiang Wang*

Key Laboratory of Bioorganic Phosphorus Chemistry and Chemical Biology (MOE), Department of Chemistry, Tsinghua University, Beijing 100084, China wangmx@mail.tsinghua.edu.cn

Table of content

1. General Information $\quad$ S1

2. Experimental Procedures and Characterization of Products S1

3. X-ray Crystallographic Data and Molecular Structures S13

$\begin{array}{lr}\text { 4. References } & \text { S19 }\end{array}$

5. Summary of Photophysical Properties of 9, 10, 15, 16, 19 and $20 \quad$ S20

6. UV/Vis and Fluorescence Spectra of 9, 10, 15, 16, 19 and $20 \quad$ S21

7. ${ }^{1} \mathrm{H}$ and ${ }^{13} \mathrm{C}$ NMR Spectra of Products $\quad$ S22 


\section{General Information}

All commercially available reagents were used as received. TLC analysis was performed on pre-coated, glass-backed silica gel plates and visualized with UV light. The silica gel (200-300 mesh) flash column chromatography was used. Anhydrous solvents (chlorobenzene, DMF, and methanol) were dried by $4 \AA$ molecular sieves. ${ }^{1} \mathrm{H}$ NMR and ${ }^{13} \mathrm{C}$ NMR spectra were recorded on a $400 \mathrm{MHz}$ or $700 \mathrm{MHz} \mathrm{NMR}$ spectrometer. Chemical shifts were reported in ppm with either tetramethylsilane or the residual solvent resonance an internal standard. Abbreviations used in the description of NMR data are as follows: chemical shift $(\delta, \mathrm{ppm})$, multiplicity $(\mathrm{s}=$ singlet, $\mathrm{d}=$ doublet, $\mathrm{t}=$ triplet, $\mathrm{q}=$ quadruplet $)$, coupling constant $(\mathrm{J}, \mathrm{Hz})$. Infrared spectra were recorded using an FT-IR spectrometer with $\mathrm{KBr}$ pellets in the $4000-400 \mathrm{~cm}^{-1}$ region. The high resolution mass spectra (HRMAS) were recorded on a mass spectrometer. UV/Vis spectra were recorded using a UV/Vis spectrophotometer. Fluorescence spectra were recorded using a fluorescence spectrophotometer. Crystallographic data were collected on a Rigaku XtaLAB Synergy $(\mathrm{Cu})$ X-ray single crystal diffractometer.

\section{Experimental Procedures and Characterization of Products}

Compounds $\mathbf{1}$ and $\mathbf{1 1}$ were synthesized according to literature procedures. ${ }^{1,2}$

Table S1. Attempted oxidation of methylene(s) of 1
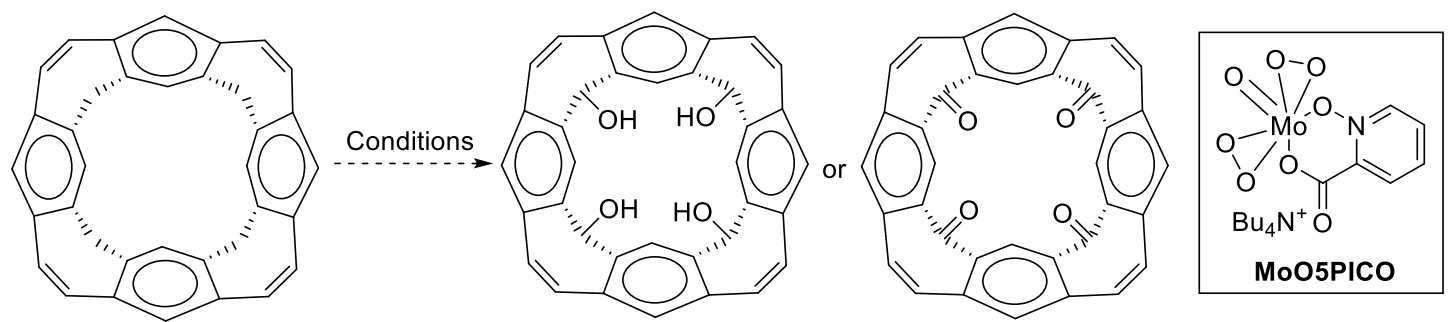

1

\begin{tabular}{lllllll}
\hline Entry & Oxidant (equiv.) & additive (equiv.) & solvent & temp. & time & result \\
\hline 1 & $\mathrm{SeO}_{2}(10)$ & - & 1,4-dioxane & Reflux & $24 \mathrm{~h}$ & N. R. \\
2 & $\mathrm{SeO}_{2}(10)$ & - & 1,4-dioxane & $160{ }^{\circ} \mathrm{C}$ & $24 \mathrm{~h}$ & N. R. \\
3 & $\mathrm{SeO}_{2}(20)$ & - & $1,4-$ dioxane & $160^{\circ} \mathrm{C}$ & $24 \mathrm{~h}$ & N. R. \\
4 & $\mathrm{SeO}_{2}(20)$ & - & PEG-400 & $240{ }^{\circ} \mathrm{C}$ & $48 \mathrm{~h}$ & N. R. \\
5 & $2 \mathrm{PyCCO}_{3}(10)$ & & DCM & $0{ }^{\circ} \mathrm{C}$ & $24 \mathrm{~h}$ & N. R. \\
\hline
\end{tabular}




\begin{tabular}{|c|c|c|c|c|c|c|}
\hline 6 & $2 \mathrm{Py} \mathrm{CrO}_{3}(20)$ & & DCM & reflux & $24 \mathrm{~h}$ & N. R. \\
\hline 7 & PCC (20) & & DCM & reflux & $24 \mathrm{~h}$ & N. R. \\
\hline 8 & $\mathrm{CrO}_{3}(20)$ & & $\mathrm{DCM}$ & reflux & $72 \mathrm{~h}$ & N. R. \\
\hline 9 & Pure $\mathrm{O}_{2}(1 \mathrm{~atm})$ & $\mathrm{Ru} @ \mathrm{Al}_{2} \mathrm{O}_{3}(0.1)^{a}$ & $o-\mathrm{DCB}^{b}$ & $170{ }^{\circ} \mathrm{C}$ & $96 \mathrm{~h}$ & N. R. \\
\hline 10 & Pure $\mathrm{O}_{2}(1 \mathrm{~atm})$ & $\mathrm{Ru} @ \mathrm{Al}_{2} \mathrm{O}_{3}(1)^{a}$ & $o-\mathrm{DCB}^{b}$ & $170{ }^{\circ} \mathrm{C}$ & $96 \mathrm{~h}$ & N. R. \\
\hline 11 & Pure $\mathrm{O}_{2}(1 \mathrm{~atm})$ & $\mathrm{Ru} @ \mathrm{Al}_{2} \mathrm{O}_{3}(1)^{a}$ & $o-\mathrm{DCB}^{b}$ & $170{ }^{\circ} \mathrm{C}$ & $96 \mathrm{~h}$ & N. R. \\
\hline 12 & Pure $\mathrm{O}_{2}(1 \mathrm{~atm})$ & $\mathrm{Ru} @ \mathrm{Al}_{2} \mathrm{O}_{3}(1)^{a}$ & $\mathrm{Ph}_{2} \mathrm{O}$ & $200{ }^{\circ} \mathrm{C}$ & $48 \mathrm{~h}$ & N. R. \\
\hline 13 & Pure $\mathrm{O}_{2}(5 \mathrm{~atm})$ & $\mathrm{Ru} @ \mathrm{Al}_{2} \mathrm{O}_{3}(1)^{a}$ & $o-\mathrm{DCB}^{b}$ & $170{ }^{\circ} \mathrm{C}$ & $72 \mathrm{~h}$ & N. R. \\
\hline 14 & Pure $\mathrm{O}_{2}(15 \mathrm{~atm})$ & $\mathrm{Ru} @ \mathrm{Al}_{2} \mathrm{O}_{3}(1)^{a}$ & $o-\mathrm{DCB}^{b}$ & $220{ }^{\circ} \mathrm{C}$ & $72 \mathrm{~h}$ & N. R. \\
\hline 15 & Pure $\mathrm{O}_{2}(15 \mathrm{~atm})$ & $\begin{array}{l}\mathrm{Ru} @ \mathrm{Al}_{2} \mathrm{O}_{3} \quad(1) \quad{ }^{a} \\
\mathrm{AcOH}(1)\end{array}$ & $o-\mathrm{DCB}^{b}$ & $220^{\circ} \mathrm{C}$ & $72 \mathrm{~h}$ & N. R. \\
\hline 16 & MoO5PICO (10) & $\mathrm{Co}(\mathrm{acac})_{2}(1)$ & DCE & Reflux & $24 \mathrm{~h}$ & N. R. \\
\hline 17 & $\mathrm{NaClO}_{2}(10)$ & NHPI (0.4) & $\mathrm{MeCN}+\mathrm{H}_{2} \mathrm{O}$ & $50^{\circ} \mathrm{C}$ & $12 \mathrm{~h}$ & N. R. \\
\hline 18 & Pure $\mathrm{O}_{2}(1 \mathrm{~atm})$ & $\begin{array}{l}\text { t-BuOK (12) } \\
\text { 18-Crown-6 (12) }\end{array}$ & DMF & $120^{\circ} \mathrm{C}$ & $24 \mathrm{~h}$ & N. R. \\
\hline 19 & $\mathrm{Pd}(\mathrm{AcO})_{2}(10)$ & & TFA & Reflux & $24 \mathrm{~h}$ & N. R. \\
\hline 20 & $\mathrm{Pd}(\mathrm{AcO})_{2}(20)$ & & TFA & Reflux & $24 \mathrm{~h}$ & N. R. \\
\hline 31 & $\mathrm{AgClO}_{4}(10)$ & & TFA & $\mathrm{rt}$ & $48 \mathrm{~h}$ & N. R. \\
\hline 22 & $\mathrm{CrO}_{3}(20)$ & & $\mathrm{AcOH}$ & reflux & $1 \mathrm{~h}$ & Mess \\
\hline 23 & $\mathrm{CrO}_{3}(20)$ & & $\mathrm{AcOH}+\mathrm{Ac}_{2} \mathrm{O}$ & reflux & $1 \mathrm{~h}$ & Mess \\
\hline 24 & $\mathrm{CrO}_{3}(20)$ & & $\mathrm{AcOH}+\mathrm{Ac}_{2} \mathrm{O}$ & $\mathrm{rt}$ & $24 \mathrm{~h}$ & Mess \\
\hline 25 & CAN (10) & & TFA & $\mathrm{rt}$ & $12 \mathrm{~h}$ & Mess \\
\hline 26 & DDQ (10) & & TFA & $\mathrm{rt}$ & $12 \mathrm{~h}$ & Mess \\
\hline 27 & DDQ (10) & & $\mathrm{AcOH}$ & $\mathrm{rt}$ & $12 \mathrm{~h}$ & Mess \\
\hline 28 & $\mathrm{KMnO}_{4}(10)$ & $\mathrm{NBu}_{4} \mathrm{PF}_{6}(4)$ & $\mathrm{DCM}+\mathrm{H}_{2} \mathrm{O}$ & $\mathrm{rt}$ & $12 \mathrm{~h}$ & Mess \\
\hline 29 & $\mathrm{NaClO}_{2}(10)$ & $\mathrm{NBu}_{4} \mathrm{PF}_{6}(4)$ & $\mathrm{DCM}+\mathrm{H}_{2} \mathrm{O}$ & $\mathrm{rt}$ & $12 \mathrm{~h}$ & N. R. \\
\hline
\end{tabular}

${ }^{a}$ Based on $\mathrm{Ru}^{3+} .{ }^{b} o$-DCB: $o$-dichlorobenzene.

\section{Synthesis of product 2}

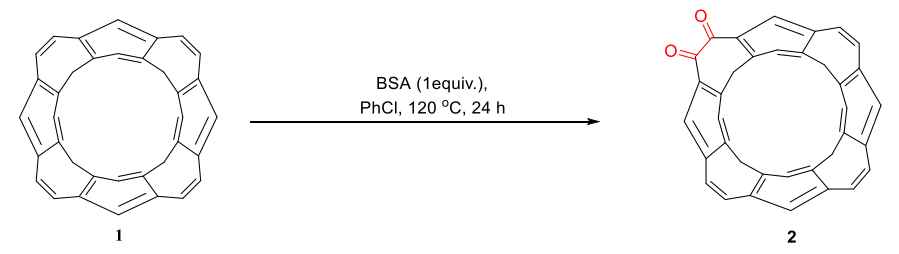

In a Schlenk tube, a mixture of $1(456.0 \mathrm{mg}, 1.0 \mathrm{mmol}, 1.0$ equiv. $)$ and $(\mathrm{PhSeO})_{2} \mathrm{O}$ (BSA) (360.1 mg, $1.0 \mathrm{mmol}, 1.0$ equiv.) in anhydrous chlorobenzene (40 mL) was heated in an oil bath at $120{ }^{\circ} \mathrm{C}$ for $24 \mathrm{~h}$. After cooling down to room temperature, the reaction mixture was concentrated using a rotary evaporator. The residue was 
chromatographed on a silica gel column (200 - 300 mesh) using DCM as mobile phase to give starting material 1 (205.2 $\mathrm{mg}, 45 \%)$ and pure product $2(253 \mathrm{mg}, 52 \%)$ as a yellow solid.

2: $\mathrm{mp}>300{ }^{\circ} \mathrm{C} ;{ }^{1} \mathrm{H}$ NMR (400 MHz, 1,1,2,2-Tetrachlorethane- $\left.d_{2}\right) \delta 7.17$ (s, 2H), 7.03 (s, 2H), $7.00(\mathrm{~s}, 2 \mathrm{H}), 6.96(\mathrm{~d}, J=11.2 \mathrm{~Hz}, 2 \mathrm{H}), 6.98(\mathrm{~s}, 2 \mathrm{H}), 6.91(\mathrm{~d}, J=11.2 \mathrm{~Hz}, 2 \mathrm{H})$, $6.86(\mathrm{~s}, 2 \mathrm{H}), 4.66(\mathrm{~d}, J=13.2 \mathrm{~Hz}, 1 \mathrm{H}), 3.78(\mathrm{~d}, J=12.0 \mathrm{~Hz}, 2 \mathrm{H}), 3.73(\mathrm{~d}, J=12.0 \mathrm{~Hz}$, $1 \mathrm{H}), 3.50(\mathrm{~d}, J=13.6 \mathrm{~Hz}, 1 \mathrm{H}), 3.49(\mathrm{~d}, J=11.6 \mathrm{~Hz}, 2 \mathrm{H}), 3.44(\mathrm{~d}, J=11.6 \mathrm{~Hz}, 1 \mathrm{H}) ;{ }^{13} \mathrm{C}$ NMR (101 MHz, 1,1,2,2-Tetrachlorethane- $\left.d_{2}\right) \delta$ 188.0, 143.2, 141.9, 140.9, 137.9, 133.3, 132.4, 131.9, 131.4, 131.3, 130.3, 129.0, 124.5, 123.8, 123.5, 40.1, 39.5, 38.2; IR (KBr) v 3010, 2953, 2905, 2847, 1678, 1609, $1596 \mathrm{~cm}^{-1}$. HRMS (APCI) Calculated for $\mathrm{C}_{36} \mathrm{H}_{23} \mathrm{O}_{2}{ }^{+}[\mathrm{M}+\mathrm{H}]^{+}:$487.1693. Found: 487.1689.

\section{Synthesis of products 3 and 4}
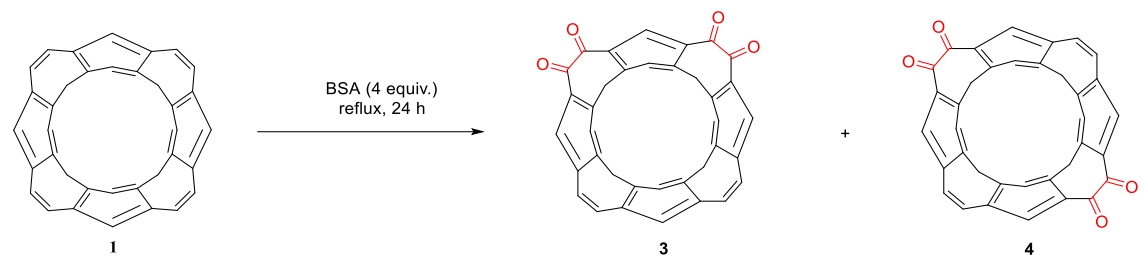

In a Schlenk tube, a mixture of 1 (456.0 mg, $1.0 \mathrm{mmol}, 1.0$ equiv.) and BSA (1.4 g, 4.0 mmol, 4.0 equiv.) in anhydrous chlorobenzene $(40 \mathrm{~mL})$ was heated to reflux for $24 \mathrm{~h}$ in an oil bath. After cooling down to room temperature, the reaction mixture was concentrated using a rotary evaporator. After preparative silica gel thin layer chromatography (DCM : ethyl acetate $=50: 1)$, pure products $3(51.7 \mathrm{mg}, 13 \%), 4$ (206.9 mg, 40\%) and 5 (120.1 mg, 22\%) were obtained. A single crystal of 3 suitable for X-ray diffraction analysis was obtained from vapor diffusion of pentane to the solution of 3 in DCM.

Product 3 was isolated as a yellow solid: $\mathrm{mp}>300{ }^{\circ} \mathrm{C} ;{ }^{1} \mathrm{H}$ NMR $(400 \mathrm{MHz}, 1,1,2,2-$ Tetrachlorethane- $\left.d_{2}\right) \delta 7.48(\mathrm{~s}, 1 \mathrm{H}), 7.28(\mathrm{~s}, 2 \mathrm{H}), 7.11(\mathrm{~s}, 1 \mathrm{H}), 7.01-7.09(\mathrm{~m}, 7 \mathrm{H}), 6.88$ $(\mathrm{s}, 1 \mathrm{H}), 4.74(\mathrm{~d}, J=13.2 \mathrm{~Hz}, 2 \mathrm{H}), 3.82(\mathrm{~d}, J=12.0 \mathrm{~Hz}, 2 \mathrm{H}), 3.61(\mathrm{~d}, J=13.2 \mathrm{~Hz}, 2 \mathrm{H})$, $3.51(\mathrm{~d}, J=11.6 \mathrm{~Hz}, 2 \mathrm{H}) ;{ }^{13} \mathrm{C}$ NMR (101 MHz, 1,1,2,2-Tetrachlorethane- $\left.d_{2}\right) \delta 187.0$, 186.7, 145.4, 143.7, 139.6, 138.9, 134.43, 134.39, 133.6, 132.2, 131.9, 131.5, 130.7, 
129.5, 125.2, 124.7, 124.4, 124.2, 39.8, 38.5; IR (KBr) v 3014, 2959, 1682, $1595 \mathrm{~cm}^{-1}$. HRMS (APCI) Calculated for $\mathrm{C}_{36} \mathrm{H}_{21} \mathrm{O}_{4}{ }^{+}[\mathrm{M}+\mathrm{H}]^{+}:$517.1434. Found: 517.1432.

4 was isolated as a yellow solid: $\mathrm{mp}>300{ }^{\circ} \mathrm{C}$; ${ }^{1} \mathrm{H}$ NMR $(400 \mathrm{MHz}, 1,1,2,2-$ Tetrachlorethane- $\left.d_{2}\right) \delta 7.20(\mathrm{~s}, 4 \mathrm{H}), 7.13(\mathrm{~s}, 4 \mathrm{H}), 6.98(\mathrm{~s}, 4 \mathrm{H}), 4.70(\mathrm{~d}, J=13.2 \mathrm{~Hz}$, $2 \mathrm{H}), 3.86(\mathrm{~d}, J=12.0 \mathrm{~Hz}, 2 \mathrm{H}), 3.60(\mathrm{~d}, J=13.2 \mathrm{~Hz}, 2 \mathrm{H}), 3.47(\mathrm{~d}, J=12.0 \mathrm{~Hz}, 2 \mathrm{H}) ;{ }^{13} \mathrm{C}$ NMR (101 MHz, 1,1,2,2-Tetrachlorethane- $\left.d_{2}\right) \delta$ 188.1, 143.0, 142.3, 133.4, 132.6, 130.6, 130.3, 130.1, 129.8, 124.6, 124.2, 40.6, 38.0; IR (KBr) v 3015, 2958, 2863, 1681, $1599 \mathrm{~cm}^{-1}$. HRMS (APCI) Calculated for $\mathrm{C}_{36} \mathrm{H}_{20} \mathrm{O}_{4}^{-}[\mathrm{M}]^{-}:$: 516.1356. Found: 516.1361.

\section{Synthesis of compound 5}
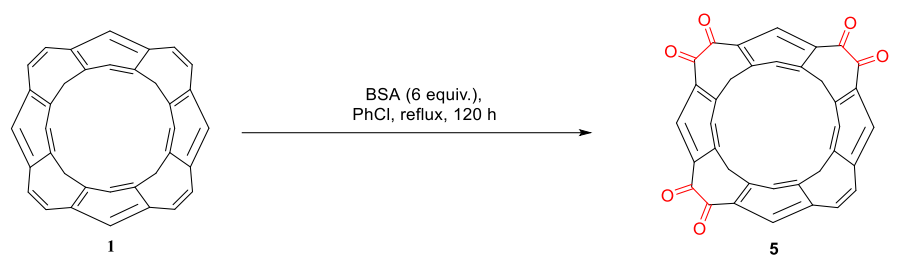

In a Schlenk tube, a mixture of 1 (456.0 mg, $1.0 \mathrm{mmol}, 1.0$ equiv.) and BSA (2.2 g, 6.0 mmol, 6.0 equiv.) in anhydrous chlorobenzene $(40 \mathrm{~mL})$ was heated at reflux for $120 \mathrm{~h}$ in an oil bath. After cooling down to room temperature, the reaction mixture was concentrated using a rotary evaporator. The residue was chromatographed on a silica gel column (200 - 300 mesh) using a mixture of DCM and ethyl acetate (V: V=25:1) as mobile phase to give pure product 5 (273.6 mg, 50\%) along with products 3 (20.7 $\mathrm{mg}, 4 \%), 4(82.7 \mathrm{mg}, 16 \%)$ and $\mathbf{6}(54.6 \mathrm{mg}, 10 \%)$. A single crystal of 5 suitable for Xray diffraction analysis was obtained from vapor diffusion of pentane to the solution of 5 in DCM.

5 was isolated as a yellow solid: $\mathrm{mp}>300{ }^{\circ} \mathrm{C} ;{ }^{1} \mathrm{H}$ NMR $\left(400 \mathrm{MHz}, \mathrm{DMSO}-d_{6}\right) \delta 7.47$ (s, 2H), 7.39 (s, 2H), 7.26 (s, 2H), 7.23 (s, 2H), 7.15 (s, 2H), 5.18 (d, J=12.8 Hz, 1H), $5.08(\mathrm{~d}, J=13.2 \mathrm{~Hz}, 2 \mathrm{H}), 4.02(\mathrm{~d}, J=11.6 \mathrm{~Hz}, 1 \mathrm{H}), 3.84$ (d, $J=12.8 \mathrm{~Hz}, 1 \mathrm{H}), 3.79$ (d, $J=12.8 \mathrm{~Hz}, 2 \mathrm{H}), 3.28(\mathrm{~d}, J=11.6 \mathrm{~Hz}, 1 \mathrm{H}) ;{ }^{13} \mathrm{C}$ NMR $\left(100 \mathrm{MHz}, \mathrm{DMSO}-d_{6}\right) \delta 186.3$, $186.1,185.2,146.1,145.8,142.7,141.1$, 134.3, 133.9, 133.7 , 133.4, 133.3, 130.5, 129.8, 127.1, 126.1, 37.0; IR (KBr) v 3025, 2963, 2872, 1685, $1599 \mathrm{~cm}^{-1}$. HRMS (APCI) Calculated for $\mathrm{C}_{36} \mathrm{H}_{18} \mathrm{O}_{6}{ }^{-}[\mathrm{M}]^{-}:$: 546.1109. Found: 546.1113.

\section{Synthesis of compound 6}



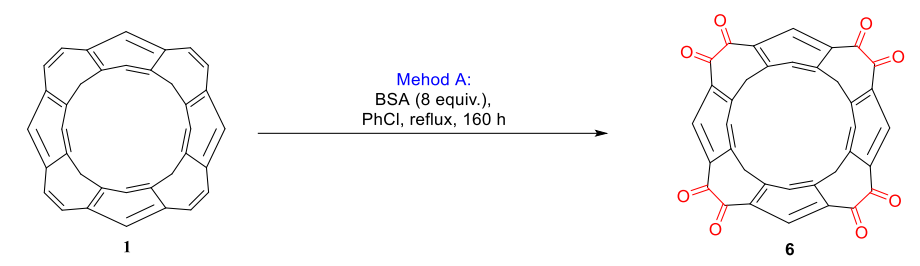

Method A : In a Schlenk tube, a mixture of 1 (456.0 mg, $1.0 \mathrm{mmol}, 1.0$ equiv.) and BSA (2.9 g, $8 \mathrm{mmol}, 8$ equiv.) in anhydrous chlorobenzene $(40 \mathrm{~mL})$ was heated at reflux for $160 \mathrm{~h}$ in an oil bath. After cooling down to room temperature, the reaction mixture was concentrated using a rotary evaporator. The residue was chromatographed on a silica gel column (200 - 300 mesh) using a mixture of DCM and ethyl acetate $(\mathrm{V}: \mathrm{V}=15: 1)$ as mobile phase to give pure products $6(92.2 \mathrm{mg}, 16 \%), 4(51.6 \mathrm{mg}, 10 \%)$ and $\mathbf{5}(273.1$ $\mathrm{mg}, 50 \%)$.
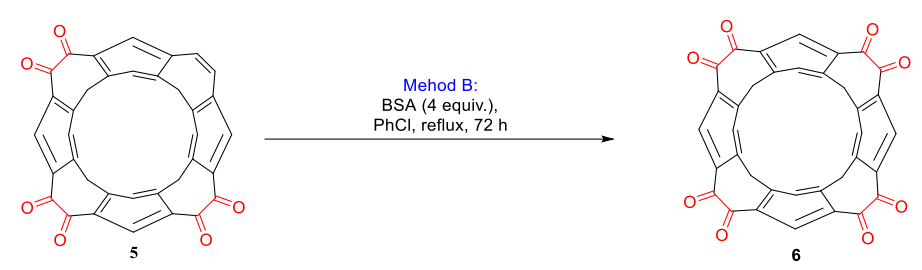

Method B: In a Schlenk tube, a mixture of 5 (546.1 mg, $1.0 \mathrm{mmol}, 1.0$ equiv.) and BSA (1.4 g, 4 mmol, 4 equiv.) in anhydrous chlorobenzene $(40 \mathrm{~mL})$ was heated at reflux for $72 \mathrm{~h}$ in an oil bath. After cooling down to room temperature, the reaction mixture was concentrated using a rotary evaporator. The residue was chromatographed on a silica gel column (200 - 300 mesh) using a mixture of DCM and ethyl acetate (V : V=15:1) as mobile phase to give pure product 6 (184.4 mg, 32\%).

6 was isolated as a yellow solid: $\mathrm{mp}>300{ }^{\circ} \mathrm{C} ;{ }^{1} \mathrm{H}$ NMR $\left(400 \mathrm{MHz}, \mathrm{DMSO}-d_{6}\right) \delta 7.58$ (s, 4H), 7.32 (s, 4H), 5.18 (d, $J=12.4 \mathrm{~Hz}, 4 \mathrm{H}), 3.88(\mathrm{~d}, J=12.4 \mathrm{~Hz}, 4 \mathrm{H}) ;{ }^{13} \mathrm{C} \mathrm{NMR}$ (100 MHz, DMSO-d $\left.d_{6}\right) \delta 185.3,146.0,134.2,133.5,127.7,37.3$; IR (KBr) v 3016, 2926, 1688, $1599 \mathrm{~cm}^{-1}$. HRMS (APCI) Calculated for $\mathrm{C}_{36} \mathrm{H}_{16} \mathrm{O}_{8}{ }^{-}[\mathrm{M}]^{-}: 576.0851$. Found: 576.0851 .

\section{Synthesis of compound 9}
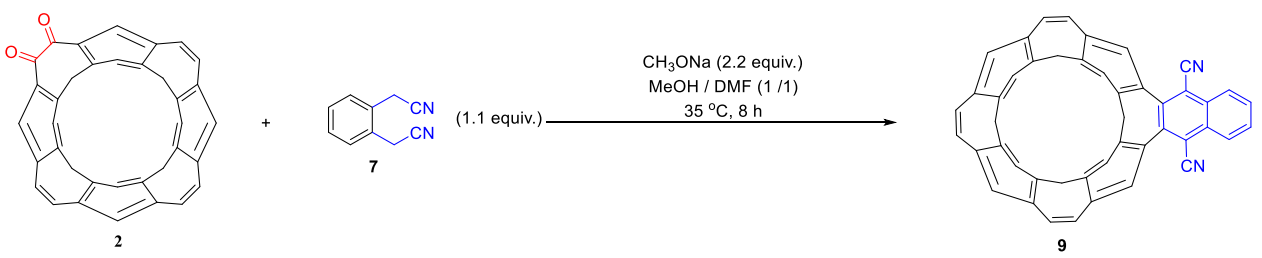

To a mixture of 2 (487.2 mg, $1.0 \mathrm{mmol}, 1.0$ equiv.), 7 (171.8 mg, $1.1 \mathrm{mmol}, 1.1$ equiv.) 
and $\mathrm{CH}_{3} \mathrm{ONa}$ (118.8 mg, $2.2 \mathrm{mmol}, 2.2$ equiv.) was added anhydrous $\mathrm{MeOH}(15 \mathrm{~mL})$ and anhydrous DMF (15 mL) under nitrogen. The resulting mixture was stirred at 35 ${ }^{\circ} \mathrm{C}$ for $8 \mathrm{~h}$. Hydrochloric acid $(0.1 \mathrm{M}, 1 \mathrm{~mL})$ was then added to quench the reaction. Brine $(100 \mathrm{~mL})$ was added, and the mixture was extracted with dichloromethane $(3 \times$ $100 \mathrm{~mL}$ ). The combined organic phase was dried over anhydrous $\mathrm{Na}_{2} \mathrm{SO}_{4}$. After filtration and removal of solvent, the residue was chromatographed on a silica gel column (200 - 300 mesh) using a mixture of DCM and ethyl acetate (V : V=100:1) as mobile phase to give pure product $9(534.8 \mathrm{mg}, 88 \%)$ as a yellow solid: $\mathrm{mp}>300{ }^{\circ} \mathrm{C}$; ${ }^{1} \mathrm{H}$ NMR (400 MHz, 1,1,2,2-Tetrachlorethane- $\left.d_{2}\right) \delta 8.46-8.43(\mathrm{~m}, 2 \mathrm{H}), 7.91-7.88(\mathrm{~m}$, 2H), 7.32 (s, 2H), 7.13 (s, 2H), $7.04(\mathrm{~s}, 2 \mathrm{H}), 6.99$ (s, 2H), 6.98 (d, J=11.6 Hz, 2H), $6.96(\mathrm{~d}, J=11.6 \mathrm{~Hz}, 2 \mathrm{H}), 6.84(\mathrm{~s}, 2 \mathrm{H}), 3.80(\mathrm{~d}, J=11.6 \mathrm{~Hz}, 2 \mathrm{H}), 3.75(\mathrm{~d}, J=11.6 \mathrm{~Hz}$, 1H), $3.61(\mathrm{~d}, J=12.4 \mathrm{~Hz}, 1 \mathrm{H}), 3.56(\mathrm{~d}, J=12.4 \mathrm{~Hz}, 1 \mathrm{H}), 3.52(\mathrm{~d}, J=11.6 \mathrm{~Hz}, 2 \mathrm{H})$, $3.48(\mathrm{~d}, J=11.2 \mathrm{~Hz}, 1 \mathrm{H}) ;{ }^{13} \mathrm{C}$ NMR (100 MHz, 1,1,2,2-Tetrachlorethane- $\left.d_{2}\right) \delta 144.9$, 142.4, 141.2, 141.0, 138.6, 132.1, 131.7, 131.6, 131.2, 130.9, 130.4, 130.2, 130.1, 129.6, 129.2, 126.1, 124.2, 123.0, 122.9, 116.9, 112.8, 39.8, 39.6, 38.7; IR (KBr) v 3012, 2954, 2904, 2845, $2219 \mathrm{~cm}^{-1}$. HRMS (APCI) Calculated for $\mathrm{C}_{46} \mathrm{H}_{27} \mathrm{~N}_{2}{ }^{+}[\mathrm{M}+\mathrm{H}]^{+}: 607.2169$. Found: 607.2174.

\section{Synthesis of compound 10}
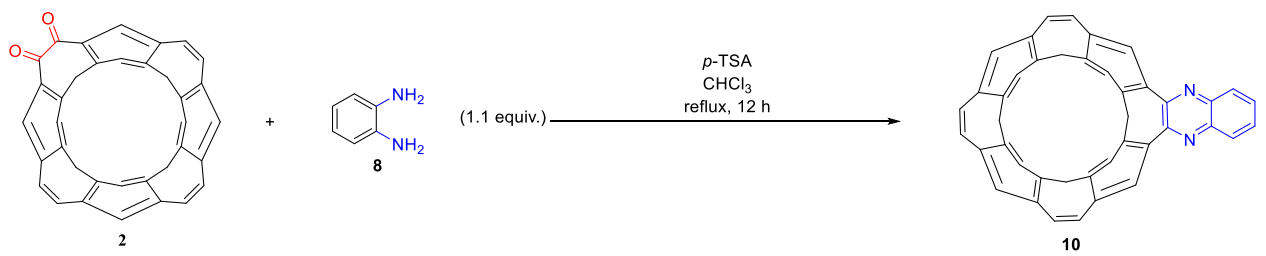

To a mixture of 2 (487.2 mg, $1.0 \mathrm{mmol}, 1.0$ equiv.), 8 (119.0 mg, $1.1 \mathrm{mmol}, 1.1$ equiv.) and $p$-TSA $(1.0 \mathrm{mg})$ was added $\mathrm{CHCl}_{3}(50 \mathrm{~mL})$. The resulting mixture was stirred at reflux using an oil bath for $12 \mathrm{~h}$. After cooling down to room temperature, the reaction mixture was concentrated using a rotary evaporator. The residue was chromatographed on a silica gel column (200 - 300 mesh) using a mixture of DCM : ethyl acetate (V : V $=50: 1)$ as mobile phase to give pure product $10(536.8 \mathrm{mg}, 96 \%)$ as a white solid : $\mathrm{mp}>300{ }^{\circ} \mathrm{C} ;{ }^{1} \mathrm{H}$ NMR (400 MHz, Chloroform-d) $\delta$ 8.23-8.20 (m, 2H), 7.81-7.78 (m, 2H), $7.36(\mathrm{~s}, 2 \mathrm{H}), 7.04(\mathrm{~s}, 2 \mathrm{H}), 6.98(\mathrm{~s}, 2 \mathrm{H}), 6.97(\mathrm{~d}, J=11.6 \mathrm{~Hz}, 2 \mathrm{H}), 6.93$ (d, $J=11.6$ 
Hz, 2H), 6.87 (s, 2H), 6.81 (s, 2H), 3.85 (d, $J=12.8 \mathrm{~Hz}, 1 \mathrm{H}), 3.76$ (d, $J=11.6 \mathrm{~Hz}, 2 \mathrm{H})$, $3.70(\mathrm{~d}, J=11.6 \mathrm{~Hz}, 1 \mathrm{H}), 3.63(\mathrm{~d}, J=12.4 \mathrm{~Hz}, 1 \mathrm{H}), 3.53(\mathrm{~d}, J=11.6 \mathrm{~Hz}, 2 \mathrm{H}), 3.45$ (d, $J=11.6 \mathrm{~Hz}, 1 \mathrm{H}) ;{ }^{13} \mathrm{C}$ NMR (100 MHz, Chloroform- $d$ ) $\delta 152.7,141.3,141.1,140.9$, 139.1, 133.1, 132.6, 132.3, 131.9, 130.8, 130.3, 130.0, 129.9, 129.3, 125.1, 123.0, 122.9, 40.2, 40.0, 38.9; IR (KBr) v 3603, 3009, 2952, 2905, 2849, 1611, $1492 \mathrm{~cm}^{-1}$. HRMS (ESI) Calculated for $\mathrm{C}_{42} \mathrm{H}_{27} \mathrm{~N}_{2}^{+}[\mathrm{M}+\mathrm{H}]^{+}$: 559.2169. Found: 559.2169.

\section{Synthesis of compound 13}
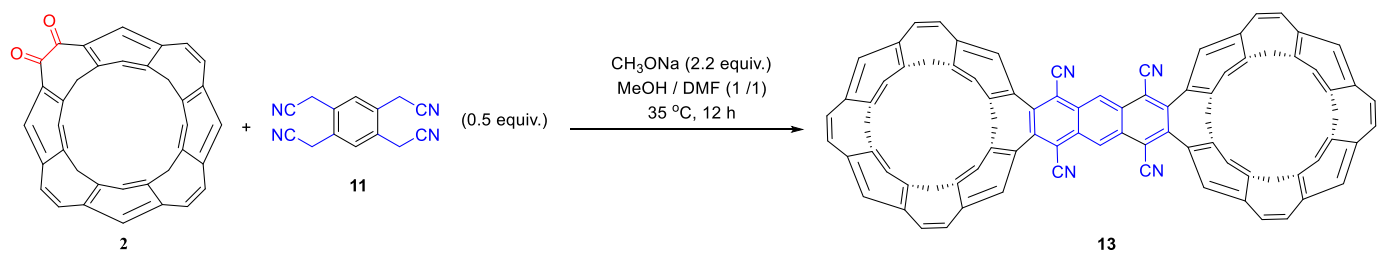

To a mixture of 2 (487.2 mg, $1.0 \mathrm{mmol}, 1.0$ equiv.), 11 (113 mg, $0.5 \mathrm{mmol}, 0.5$ equiv.) and $\mathrm{CH}_{3} \mathrm{ONa}$ (118.8 mg, $2.2 \mathrm{mmol}, 2.2$ equiv.) was added anhydrous $\mathrm{MeOH}(15 \mathrm{~mL})$ and anhydrous DMF $(15 \mathrm{~mL})$ under nitrogen. The resulting mixture was stirred at 35 ${ }^{\circ} \mathrm{C}$ using an oil bath for $12 \mathrm{~h}$. Hydrochloric acid $(0.1 \mathrm{M}, 1 \mathrm{~mL})$ was added to quench the reaction. Brine $(100 \mathrm{~mL})$ was added, and the mixture was extracted with dichloromethane $(3 \times 100 \mathrm{~mL})$. The combined organic phase was dried over anhydrous $\mathrm{Na}_{2} \mathrm{SO}_{4}$. After filtration and removal of solvent, the residue was chromatographed on a silica gel column (200 - 300 mesh) using DCM as mobile phase to give pure product 13 (260.8 mg, 46\%) as a orange solid: $\mathrm{mp}>300{ }^{\circ} \mathrm{C}$; ${ }^{1} \mathrm{H}$ NMR (400 MHz, Chloroformd) $\delta 9.54(\mathrm{~s}, 2 \mathrm{H}), 7.36(\mathrm{~s}, 4 \mathrm{H}), 7.10(\mathrm{~s}, 4 \mathrm{H}), 6.99(\mathrm{~s}, 4 \mathrm{H}), 6.97(\mathrm{~s}, 8 \mathrm{H}), 6.92(\mathrm{~s}, 4 \mathrm{H}), 6.84$ (s, 4H), $3.77(\mathrm{~d}, J=11.6 \mathrm{~Hz}, 4 \mathrm{H}), 3.71$ (d, $J=12.8 \mathrm{~Hz}, 2 \mathrm{H}), 3.68$ (d, $J=12.8 \mathrm{~Hz}, 2 \mathrm{H})$, $3.59(\mathrm{~d}, J=12.0 \mathrm{~Hz}, 2 \mathrm{H}), 3.50(\mathrm{~d}, J=12.0 \mathrm{~Hz}, 4 \mathrm{H}), 3.47(\mathrm{~d}, J=11.6 \mathrm{~Hz}, 2 \mathrm{H}) ;{ }^{13} \mathrm{C}$ NMR (100 MHz, Chloroform- $d$ ) $\delta$ 145.7, 145.0, 142.2, 141.4, 138.6, 133.0 , 132.5, 131.9, 131.6, 131.1, 130.43, 130.38, 129.6, 129.2, 125.3, 125.2, 123.5, 123.2, 116.3, 113.3, 40.3, 40.1, 39.3. IR (KBr) v 3008, 2949, 2918, 2847, $2221 \mathrm{~cm}^{-1}$. HRMS (MALDI) Calculated for $\mathrm{C}_{86} \mathrm{H}_{47} \mathrm{~N}_{4}^{+}[\mathrm{M}+\mathrm{H}]^{+}:$1133.3639. Found: 1133.3640 .

\section{Synthesis of compound 14}



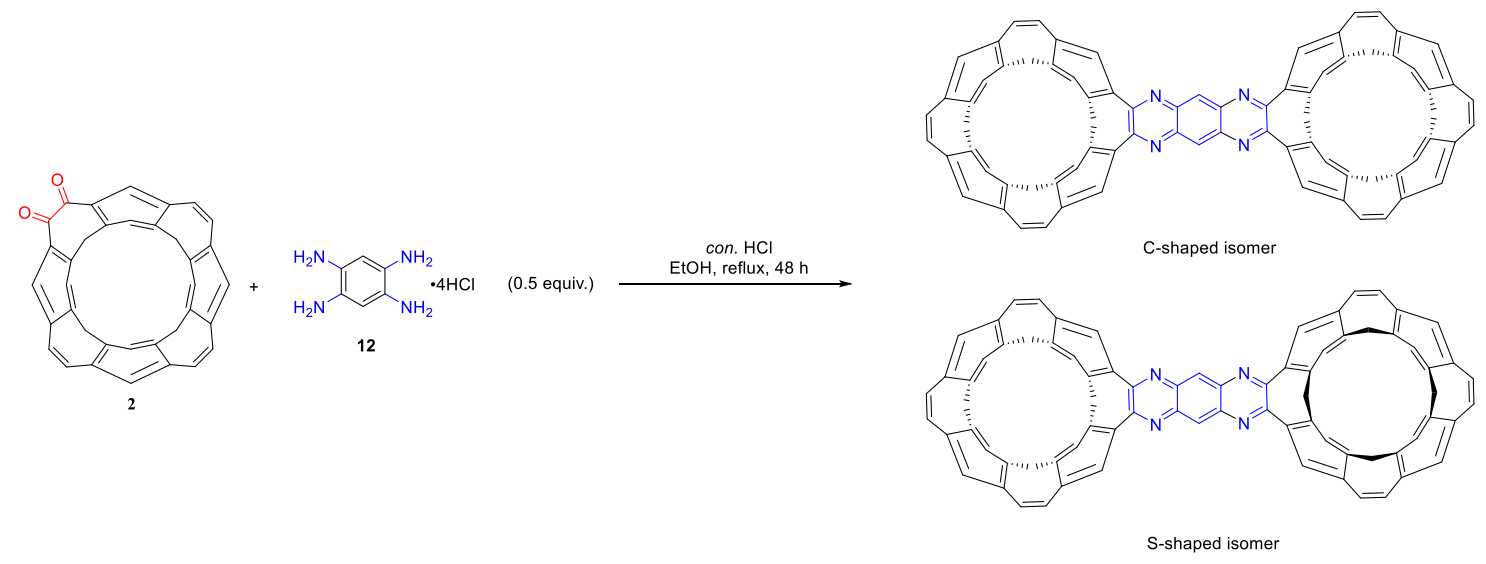

To a mixture of 2 (487.2 mg, $1.0 \mathrm{mmol}, 1.0$ equiv.), and 12 (142 mg, $0.5 \mathrm{mmol}, 0.5$ equiv.) was added EtOH $(50 \mathrm{~mL})$ followed by con. Hydrochloric acid $(3.3 \mathrm{ml})$. The resulting mixture was stirred at reflux using an oil bath for $48 \mathrm{~h}$ in an oil bath. After cooling down to room temperature, saturated $\mathrm{Na}_{2} \mathrm{CO}_{3}$ solution was added to adjust $\mathrm{pH}$ to neutral. Brine $(100 \mathrm{~mL})$ was added, and the mixture was extracted with dichloromethane $(3 \times 100 \mathrm{~mL})$. The combined organic phase was dried over anhydrous $\mathrm{Na}_{2} \mathrm{SO}_{4}$. After filtration and removal of solvent, the residue was chromatographed on a silica gel column (200 - 300 mesh) using DCM as mobile phase to give pure C-shaped isomer (114.4 mg, 22\%) and S-shaped isomer (114.4 mg, 22\%). A single crystal of Cshaped 14 suitable for X-ray diffraction analysis was obtained from vapor diffusion of hexane to the solution of C-shaped 14 in 1,1,2,2-tetrachloroethane.

C-shaped isomer was obtained as a yellow solid: $\mathrm{mp}>300{ }^{\circ} \mathrm{C} ;{ }^{1} \mathrm{H}$ NMR $(400 \mathrm{MHz}$, 1,1,2,2-Tetrachlorethane- $\left.d_{2}\right) \delta 9.07(\mathrm{~s}, 2 \mathrm{H}), 7.45(\mathrm{~s}, 4 \mathrm{H}), 7.10(\mathrm{~s}, 4 \mathrm{H}), 7.07(\mathrm{~s}, 4 \mathrm{H}), 7.03-$ $6.93(\mathrm{~m}, 12 \mathrm{H}), 6.84(\mathrm{~s}, 4 \mathrm{H}), 3.97(\mathrm{~d}, J=12.8 \mathrm{~Hz}, 2 \mathrm{H}), 3.83(\mathrm{~d}, J=11.6 \mathrm{~Hz}, 4 \mathrm{H}), 3.76$ $(\mathrm{d}, J=11.2 \mathrm{~Hz}, 2 \mathrm{H}), 3.67(\mathrm{~d}, J=13.6 \mathrm{~Hz}, 2 \mathrm{H}), 3.56(\mathrm{~d}, J=11.2 \mathrm{~Hz}, 4 \mathrm{H}), 3.46(\mathrm{~d}, J=$ $11.2 \mathrm{~Hz}, 2 \mathrm{H})$; IR (KBr) v 2921, 2851, 1698, $1660 \mathrm{~cm}^{-1}$. HRMS (MALDI) Calculated for $\mathrm{C}_{78} \mathrm{H}_{47} \mathrm{~N}_{4}^{+}[\mathrm{M}+\mathrm{H}]^{+}$: 1039.3795. Found: 1039.3797.

S-shaped isomer was isolated as a yellow solid: $\mathrm{mp}>300{ }^{\circ} \mathrm{C} ;{ }^{1} \mathrm{H}$ NMR $(400 \mathrm{MHz}$, 1,1,2,2-Tetrachlorethane- $\left.d_{2}\right) \delta 9.04(\mathrm{~s}, 2 \mathrm{H}), 7.44(\mathrm{~s}, 4 \mathrm{H}), 7.08(\mathrm{~s}, 4 \mathrm{H}), 7.07(\mathrm{~s}, 4 \mathrm{H}), 7.02$ (d, $J=12.4 \mathrm{~Hz}, 4 \mathrm{H}), 6.97(\mathrm{~d}, J=12.0 \mathrm{~Hz}, 4 \mathrm{H}), 6.96(\mathrm{~s}, 4 \mathrm{H}), 6.83(\mathrm{~s}, 4 \mathrm{H}), 4.00$ (d, $J=$ $11.6 \mathrm{~Hz}, 2 \mathrm{H}), 3.82(\mathrm{~d}, J=11.6 \mathrm{~Hz}, 4 \mathrm{H}), 3.76(\mathrm{~d}, J=11.2 \mathrm{~Hz}, 2 \mathrm{H}), 3.65(\mathrm{~d}, J=12.0 \mathrm{~Hz}$, 2H), $3.56(\mathrm{~d}, J=11.2 \mathrm{~Hz}, 4 \mathrm{H}), 3.46$ (d, $J=11.6 \mathrm{~Hz}, 2 \mathrm{H})$; IR (KBr) v 2958, 2923, 2852, 
1699, $1610 \mathrm{~cm}^{-1}$. HRMS (MALDI) Calculated for $\mathrm{C}_{78} \mathrm{H}_{47} \mathrm{~N}_{4}^{+}[\mathrm{M}+\mathrm{H}]^{+}: 1039.3795$. Found: 1039.3795.

\section{Synthesis of compound 15}
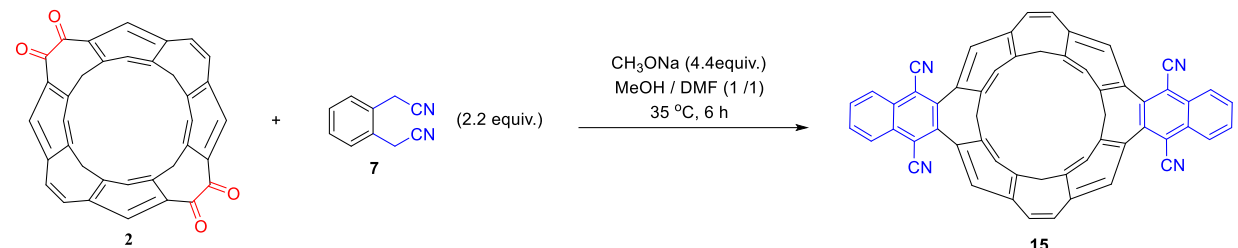

To a mixture of 2 (487.2 mg, $1.0 \mathrm{mmol}, 1.0$ equiv.), 7 (343.6 mg, $2.2 \mathrm{mmol}, 2.2$ equiv.) and $\mathrm{CH}_{3} \mathrm{ONa}$ (237.6 mg, $4.4 \mathrm{mmol}, 4.4$ equiv.) was added anhydrous $\mathrm{MeOH}(15 \mathrm{~mL})$ and anhydrous DMF $(15 \mathrm{~mL})$ under nitrogen. The resulting mixture was stirred at 35 ${ }^{\circ} \mathrm{C}$ using an oil bath for $8 \mathrm{~h}$. Hydrochloric acid $(0.1 \mathrm{M}, 1 \mathrm{~mL})$ was added to quench the reaction. Brine $(100 \mathrm{~mL})$ was added, and the mixture was extracted with dichloromethane $(3 \times 100 \mathrm{~mL})$. The combined organic phase was dried over anhydrous $\mathrm{Na}_{2} \mathrm{SO}_{4}$. After filtration and removal of solvent, the residue was chromatographed on a silica gel column (200 - 300 mesh) using a mixture of DCM and ethyl acetate (V : V = $50: 1)$ as mobile phase to give pure product $15(534.8 \mathrm{mg}, 88 \%)$ as a yellow solid: $\mathrm{mp}>300{ }^{\circ} \mathrm{C} ;{ }^{1} \mathrm{H}$ NMR $\left(400 \mathrm{MHz}\right.$, Methylene Chloride- $\left.d_{2}\right) \delta 8.47-8.44(\mathrm{~m}, 4 \mathrm{H}), 7.91-$ $7.88(\mathrm{~m}, 4 \mathrm{H}), 7.33(\mathrm{~s}, 4 \mathrm{H}), 7.26(\mathrm{~s}, 4 \mathrm{H}), 7.04(\mathrm{~s}, 4 \mathrm{H}), 3.90(\mathrm{~d}, J=12.0 \mathrm{~Hz}, 2 \mathrm{H}), 3.69(\mathrm{~d}$, $J=12.4 \mathrm{~Hz}, 2 \mathrm{H}), 3.63(\mathrm{~d}, J=12.4 \mathrm{~Hz}, 2 \mathrm{H}), 3.57(\mathrm{~d}, J=12.0 \mathrm{~Hz}, 2 \mathrm{H}) ;{ }^{13} \mathrm{C}$ NMR $(100$ MHz, Methylene Chloride- $\left.d_{2}\right) \delta 146.1,142.8,140.8,132.7,131.9,130.9,130.49$, 130.45, 130.1, 126.6, 124.1, 117.0, 113.3, 40.4, 39.2; IR (KBr) v 3014, 2955, 2223, $1608 \mathrm{~cm}^{-1}$. HRMS (APCI) Calculated for $\mathrm{C}_{56} \mathrm{H}_{29} \mathrm{~N}_{4}{ }^{+}[\mathrm{M}+\mathrm{H}]^{+}:$757.2388. Found: 757.2376 .

\section{Synthesis of compound 16}
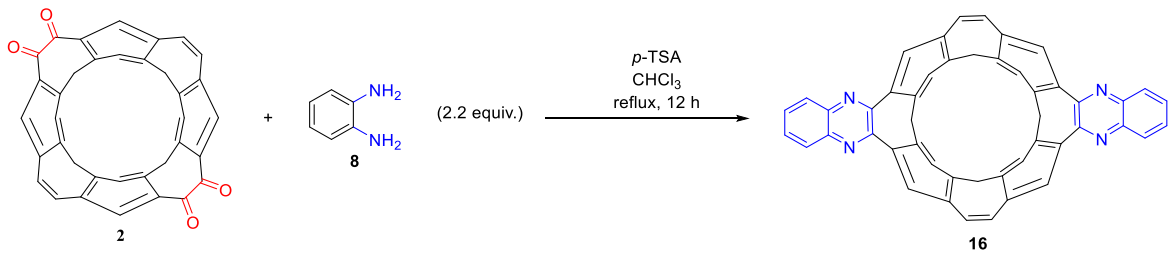

To a mixture of 2 (487.2 mg, $1.0 \mathrm{mmol}, 1.0$ equiv.), 8 (238.0 mg, $2.2 \mathrm{mmol}, 2.2$ equiv.) and $p$-TSA $(1.0 \mathrm{mg})$ was added $\mathrm{CHCl}_{3}(50 \mathrm{~mL})$. The resulting mixture was stirred at reflux for $12 \mathrm{~h}$ in an oil bath. After cooling down to room temperature, the reaction 
mixture was concentrated using a rotary evaporator. The residue was chromatographed on a silica gel column (200 - 300 mesh) using a mixture of DCM and ethyl acetate (V : $\mathrm{V}=25: 1)$ as mobile phase to give pure product $16(621.5 \mathrm{mg}, 94 \%)$ as a white solid : $\mathrm{mp}>300{ }^{\circ} \mathrm{C} ;{ }^{1} \mathrm{H}$ NMR (400 MHz, Chloroform- $d$ ) $\delta$ 8.22-8.20 (m, 4H), 7.81-7.78 (m, 4H), 7.39 (s, 4H), 7.14 (s, 4H), 7.05 (s, 4H), 3.87 (d, $J=12.4 \mathrm{~Hz}, 2 \mathrm{H}), 3.86(\mathrm{~d}, J=12.0$ $\mathrm{Hz}, 2 \mathrm{H}), 3.68(\mathrm{~d}, J=12.8 \mathrm{~Hz}, 2 \mathrm{H}), 3.61(\mathrm{~d}, J=11.6 \mathrm{~Hz}, 2 \mathrm{H}) ;{ }^{13} \mathrm{C}$ NMR (100 MHz, Chloroform- $d$ ) $\delta 152.6,144.2,141.3,140.7,133.1,132.8,130.7,130.14,130.07,130.0$, 129.3, 123.5, 123.4, 40.3, 38.9; IR (KBr) v 3058, 3012, 2951, 2915, 2861, $1607 \mathrm{~cm}^{-1}$. HRMS (ESI) Calculated for $\mathrm{C}_{48} \mathrm{H}_{29} \mathrm{~N}_{4}^{+}[\mathrm{M}+\mathrm{H}]^{+}$: 661.2387. Found: 661.2385.

\section{Synthesis of compound 17}

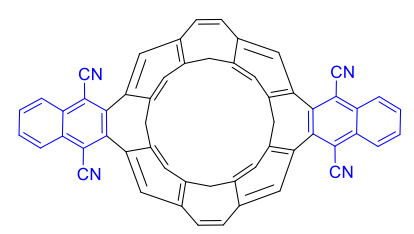

15

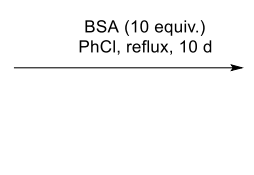

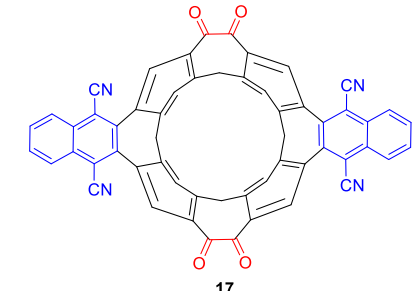

17

In a Schlenk tube, a mixture of 15 (378.6 mg, $0.5 \mathrm{mmol}, 0.5$ equiv.) and BSA (1.8g, 5.0 mmol, 5.0 equiv.) in anhydrous chlorobenzene $(80 \mathrm{~mL})$ was heated at reflux for $10 \mathrm{~d}$ in an oil bath. After cooling down to room temperature, the reaction mixture was concentrated using a rotary evaporator. The residue was chromatographed on a silica gel column (200 - 300 mesh) using a mixture of DCM and ethyl acetate (V : V=20:1) as mobile phase to give pure product $17(259.6 \mathrm{mg}, 72 \%)$ as a yellow solid: $\mathrm{mp}>300$ ${ }^{\circ} \mathrm{C}$; ${ }^{1} \mathrm{H}$ NMR (400 MHz, 1,1,2,2-Tetrachlorethane- $\left.d_{2}\right) \delta$ 8.51-8.49 (m, 4H), 7.99-7.97 (m, 4H), $7.80(\mathrm{~s}, 4 \mathrm{H}), 7.40$ (s, 4H), 4.90 (d, $J=13.2 \mathrm{~Hz}, 2 \mathrm{H}), 3.86$ (d, $J=12.4 \mathrm{~Hz}, 2 \mathrm{H})$, $3.80(\mathrm{~d}, J=13.6 \mathrm{~Hz}, 2 \mathrm{H}), 3.75(\mathrm{~d}, J=12.4 \mathrm{~Hz}, 2 \mathrm{H}) ;{ }^{13} \mathrm{C} \mathrm{NMR}(175 \mathrm{MHz}, 1,1,2,2-$ Tetrachlorethane- $\left.d_{2}\right) \delta 186.2,148.8,142.4,139.9,134.5,133.5,131.5,131.3,131.1$, 126.4, 125.5, $115.9,113.5,39.5,38.3$. IR (KBr) v 2925, 2867, 2223, 1681, $1603 \mathrm{~cm}^{-1}$. HRMS (APCI) Calculated for $\mathrm{C}_{56} \mathrm{H}_{25} \mathrm{O}_{4} \mathrm{~N}_{4}{ }^{+}[\mathrm{M}+\mathrm{H}]^{+}$: 817.1870. Found: 817.1853.

\section{Synthesis of compound 18}




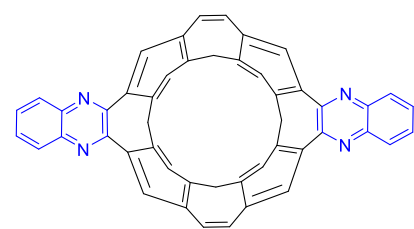

16

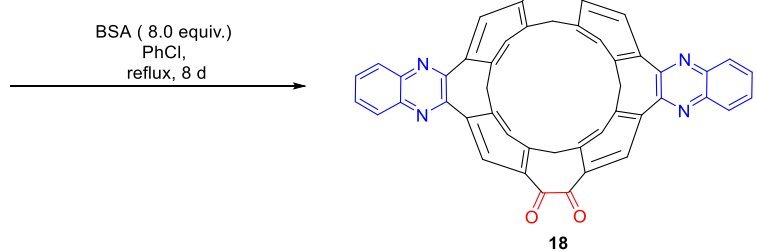

In a Schlenk tube, a mixture of 16 (330.6 mg, $0.5 \mathrm{mmol}, 0.5$ equiv.) and BSA (1.8g, 5.0 mmol, 5.0 equiv.) in anhydrous chlorobenzene $(80 \mathrm{~mL})$ was heated at reflux for $8 \mathrm{~d}$ in an oil bath. After cooling down to room temperature, the reaction mixture was concentrated using a rotary evaporator. The residue was chromatographed on a silica gel column (200 - 300 mesh) using a mixture of DCM and ethyl acetate $(\mathrm{V}: \mathrm{V}=10: 1)$ as mobile phase to give pure product $18(346.2 \mathrm{mg}, 96 \%)$ as a yellow solid: $\mathrm{mp}>300$ ${ }^{\circ} \mathrm{C}$; ${ }^{1} \mathrm{H}$ NMR (400 MHz, Chloroform- $d$ ) $\delta$ 8.25-8.22 (m, 4H), 7.90 (s, 4H), 7.88-7.85 (m, 4H), $7.33(\mathrm{~s}, 4 \mathrm{H}), 4.96(\mathrm{~d}, J=13.2 \mathrm{~Hz}, 2 \mathrm{H}), 3.97(\mathrm{~d}, J=12.0 \mathrm{~Hz}, 2 \mathrm{H}), 3.88(\mathrm{~d}, J=$ 13.6 Hz, 2H), 3.80 (d, $J=12.4 \mathrm{~Hz}, 2 \mathrm{H}) .{ }^{13} \mathrm{C}$ NMR (100 MHz, Chloroform- $d$ ) $\delta$ 187.2, 150.1, 147.2, 142.5, 141.8, 135.2, 134.7, 134.4, 131.1, 129.6, 125.5, 39.7, 38.7. IR (KBr) $v$ 2924, 2853, 1681, $1602 \mathrm{~cm}^{-1}$. HRMS (APCI) Calculated for $\mathrm{C}_{48} \mathrm{H}_{25} \mathrm{O}_{4} \mathrm{~N}_{4}^{+}[\mathrm{M}+\mathrm{H}]^{+}$: 721.1870. Found: 721.1861.

\section{Synthesis of compound 19}
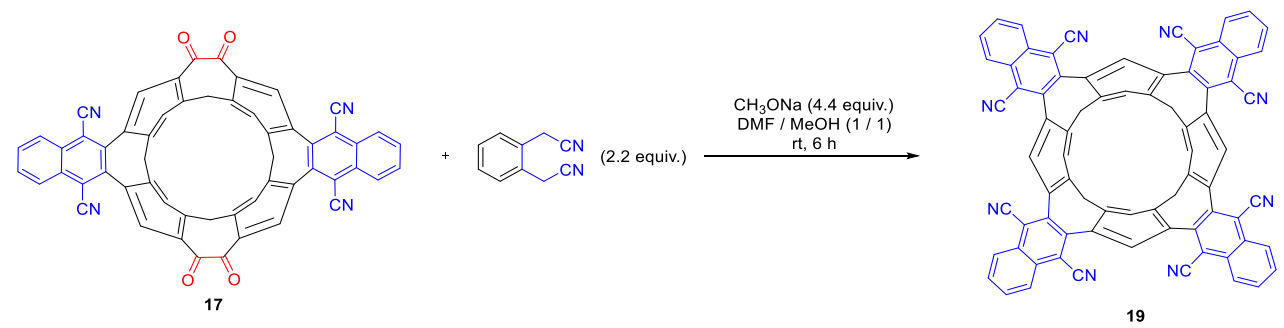

To a mixture of 17 (408.6 mg, $0.5 \mathrm{mmol}, 1.0$ equiv.), 7 (171.8 $\mathrm{mg}, 1.1 \mathrm{mmol}, 2.2$ equiv.) and $\mathrm{CH}_{3} \mathrm{ONa}$ (118.8 mg, $2.2 \mathrm{mmol}, 4.4$ equiv.) was added anhydrous $\mathrm{MeOH}(15 \mathrm{~mL})$ and anhydrous DMF (15 mL) under nitrogen. The resulting mixture was stirred at room temperature for $6 \mathrm{~h}$. Hydrochloric acid $(0.1 \mathrm{M}, 1 \mathrm{~mL})$ was added to quench the reaction. Brine $(100 \mathrm{~mL})$ was added, and the mixture was extracted with dichloromethane $(3 \times$ $100 \mathrm{~mL}$ ). The combined organic phase was dried over anhydrous $\mathrm{Na}_{2} \mathrm{SO}_{4}$. After filtration and removal of solvent, the residue was chromatographed on a silica gel column (200 - 300 mesh) using a mixture of DCM and ethyl acetate (V: V=20:1) as mobile phase to give pure product $19(317.2 \mathrm{mg}, 60 \%)$ as a white solid: $\mathrm{mp}>300{ }^{\circ} \mathrm{C}$; 
${ }^{1} \mathrm{H}$ NMR (400 MHz, 1,1,2,2-Tetrachlorethane- $\left.d_{2}\right) \delta 8.58-8.56(\mathrm{~m}, 8 \mathrm{H}), 7.96-7.94(\mathrm{~m}$, $8 \mathrm{H}), 7.83(\mathrm{~s}, 4 \mathrm{H}), 7.51(\mathrm{~s}, 4 \mathrm{H}), 3.88(\mathrm{~d}, J=12.8 \mathrm{~Hz}, 4 \mathrm{H}), 3.82(\mathrm{~d}, J=12.8 \mathrm{~Hz}, 4 \mathrm{H}) ;{ }^{13} \mathrm{C}$ NMR (100 MHz, 1,1,2,2-Tetrachlorethane- $\left.d_{2}\right) \delta$ 146.0, 140.4, 133.5, 131.8, 131.1, 130.7, 126.5, 124.4, 116.0, 114.1, 39.4; IR (KBr) v 2922, 2851, 2224, 1659, $1633 \mathrm{~cm}^{-1}$. HRMS (APCI) Calculated for $\mathrm{C}_{76} \mathrm{H}_{33} \mathrm{~N}_{8}{ }^{+}[\mathrm{M}+\mathrm{H}]^{+}:$1057.2823. Found: 1057.2831. A single crystal of $\mathbf{1 9}$ suitable for X-ray diffraction analysis was obtained from vapor diffusion of hexane to the solution of $\mathbf{1 9}$ in 1,2-dichloroethane.

\section{Synthesis of compound 20}
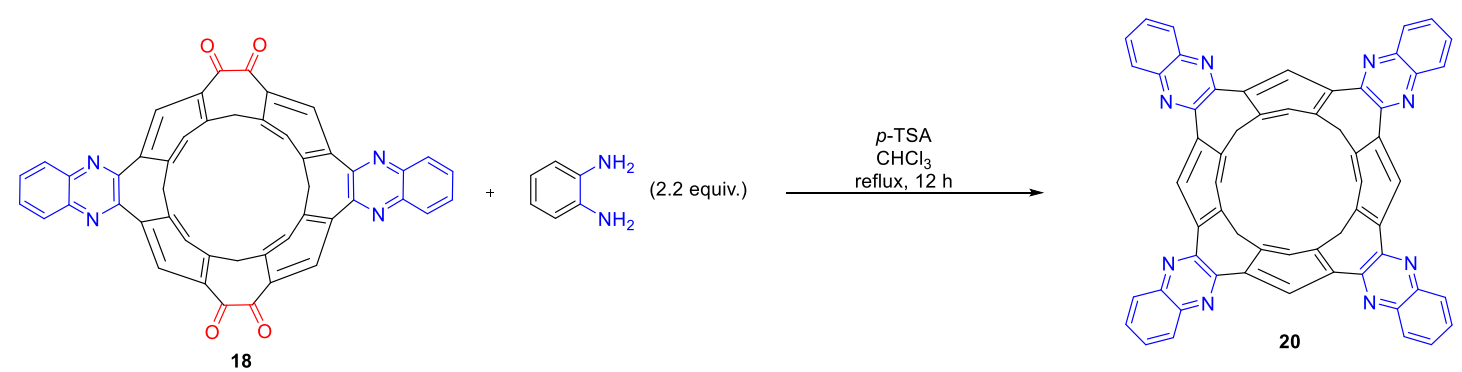

To a mixture of 18 (360.6 mg, $0.5 \mathrm{mmol}, 1.0$ equiv.), 8 (119.0 mg, $1.1 \mathrm{mmol}, 2.2$ equiv.) and $p$-TSA $(1.0 \mathrm{mg})$ was added $\mathrm{CHCl}_{3}(50 \mathrm{~mL})$. The resulting mixture was stirred at reflux for $12 \mathrm{~h}$ in an oil bath. After cooling down to room temperature, the reaction mixture was concentrated using a rotary evaporator. The residue was chromatographed on a silica gel column (200 - 300 mesh) using a mixture of DCM and ethyl acetate (V : $\mathrm{V}=5: 1)$ as mobile phase to give pure product $\mathbf{2 0}(386.4 \mathrm{mg}, 89 \%)$ as a white solid : $\mathrm{mp}>300{ }^{\circ} \mathrm{C} ;{ }^{1} \mathrm{H}$ NMR (400 MHz, Chloroform- $d$ ) $\delta 8.28-8.24(\mathrm{~m}, 8 \mathrm{H}), 8.04(\mathrm{~s}, 4 \mathrm{H})$, 7.79 - 7.76 (m, 8H), 7.36 (s, 4H), 4.07 (d, $J=12.0 \mathrm{~Hz}, 4 \mathrm{H}), 3.87$ (d, $J=12.4 \mathrm{~Hz}, 4 \mathrm{H})$. ${ }^{13} \mathrm{C}$ NMR (100 MHz, Chloroform- $d$ ) $\delta 151.4,143.9$, 141.3 , 134.2 , 134.0 , 129.9 , $129.3,123.8,38.9$. IR (KBr) v 3059, 2956, 1696, $1606 \mathrm{~cm}^{-1}$. HRMS (ESI) Calculated for $\mathrm{C}_{60} \mathrm{H}_{33} \mathrm{~N}_{8}^{+}[\mathrm{M}+\mathrm{H}]^{+}$: 865.2828, Found: 865.2824. 
3. Crystal data structure refinement for X-ray molecular structures of 3, 5, 14 and 19

\begin{tabular}{|c|c|c|c|c|}
\hline Identification code & 3 & 5 & $\mathbf{1 4}$ (C-typed isomer) & 19 \\
\hline Empirical formula & $\mathrm{C} 36 \mathrm{H} 20 \mathrm{O} 4$ & C36 H18 O6 & $\mathrm{C} 43 \mathrm{H} 27 \mathrm{Cl} 8 \mathrm{~N} 2$ & $\mathrm{C} 80 \mathrm{H} 40 \mathrm{Cl} 4 \mathrm{~N} 8$ \\
\hline Formula weight & 516.52 & 546.50 & 855.26 & 1255.00 \\
\hline Temperature & 109(1) K & $110(1) \mathrm{K}$ & $170.00(13) \mathrm{K}$ & $169.99(13) \mathrm{K}$ \\
\hline Wavelength & $1.54184 \AA$ & $1.54184 \AA$ & $1.54184 \AA$ & $1.54184 \AA$ \\
\hline Crystal system & Monoclinic & Monoclinic & Monoclinic & Monoclinic \\
\hline Space group & P 1211 & P $121 / \mathrm{n} 1$ & I 1 2/a 1 & P $121 / \mathrm{c} 1$ \\
\hline Unit cell dimensions & $\begin{array}{l}a=10.5043(4) \AA, \\
b=20.6891(5) \AA, \\
c=12.7564(4) \AA \\
\alpha=90^{\circ}, \\
\beta=107.767(4)^{\circ}, \\
\gamma=90^{\circ}\end{array}$ & $\begin{array}{l}a=10.5406(3) \AA, \\
b=21.3491(7) \AA, \\
c=11.5453(4) \AA, \\
\alpha=90^{\circ}, \\
\beta=91.090(3)^{\circ}, \\
\gamma=90^{\circ}\end{array}$ & $\begin{array}{l}a=20.4246(12) \AA, \\
b=18.4661(15) \AA, \\
c=22.1441(15) \AA, \\
\alpha=90^{\circ}, \\
\beta=103.279, \\
\gamma=90^{\circ}\end{array}$ & $\begin{array}{l}a=27.9614(5) \AA, \\
b=17.1911(3) \AA, \\
c=15.6409(3) \AA, \\
\alpha=90^{\circ}, \\
\beta=104.007(2)^{\circ}, \\
\gamma=90^{\circ}\end{array}$ \\
\hline Volume & $2640.05(16) \AA^{3}$ & $2597.60(14) \AA^{3}$ & $8128.6(10) \AA^{3}$ & $7294.8(2) \AA^{3}$ \\
\hline $\mathrm{Z}$ & 4 & 4 & 8 & 4 \\
\hline Density (calculated) & $1.300 \mathrm{Mg} / \mathrm{m}^{3}$ & $1.397 \mathrm{Mg} / \mathrm{m}^{3}$ & $1.398 \mathrm{Mg} / \mathrm{m}^{3}$ & $1.143 \mathrm{Mg} / \mathrm{m}^{3}$ \\
\hline Absorption coefficient & $0.677 \mathrm{~mm}^{-1}$ & $0.781 \mathrm{~mm}^{-1}$ & $5.329 \mathrm{~mm}^{-1}$ & $1.840 \mathrm{~mm}^{-1}$ \\
\hline $\mathrm{F}(000)$ & 1072 & 1128 & 3480 & 2576 \\
\hline
\end{tabular}




\begin{tabular}{|c|c|c|c|c|}
\hline Crystal size & $0.15 \times 0.10 \times 0.08 \mathrm{~mm}^{3}$ & $0.12 \times 0.1 \times 0.02 \mathrm{~mm}^{3}$ & $0.12 \times 0.08 \times 0.05 \mathrm{~mm}^{3}$ & $0.2 \times 0.15 \times 0.02 \mathrm{~mm}^{3}$ \\
\hline Theta range for data collection & 3.638 to $71.561^{\circ}$. & 4.142 to $71.525^{\circ}$. & 3.151 to $75.624^{\circ}$. & 3.043 to $75.512^{\circ}$. \\
\hline Index ranges & $\begin{array}{l}-12<=\mathrm{h}<=12,-16<=\mathrm{k}<=25,- \\
15<=\mathrm{l}<=14\end{array}$ & $\begin{array}{l}-9<=\mathrm{h}<=12,-25<=\mathrm{k}<=21,- \\
13<=1<=13\end{array}$ & $\begin{array}{l}-24<=\mathrm{h}<=25,-22<=\mathrm{k}<=22,- \\
27<=\mathrm{l}<=27\end{array}$ & $\begin{array}{c}-35<=\mathrm{h}<=34,-20<=\mathrm{k}<=21,- \\
19<=\mathrm{l}<=17\end{array}$ \\
\hline Reflections collected & 10445 & 9822 & 28338 & 50913 \\
\hline Independent reflections & $7109[\mathrm{R}$ (int) $=0.0408]$ & $4897[\mathrm{R}(\mathrm{int})=0.0374]$ & $8021[\mathrm{R}(\mathrm{int})=0.1316]$ & $14578[\mathrm{R}(\mathrm{int})=0.0620]$ \\
\hline Completeness & $99.5 \%\left(\right.$ to theta $\left.=67.684^{\circ}\right)$ & $99.7 \%\left(\right.$ to theta $\left.=67.684^{\circ}\right)$ & $99.7 \%\left(\right.$ to theta $\left.=67.684^{\circ}\right)$ & $99.8 \%\left(\right.$ to theta $\left.=67.684^{\circ}\right)$ \\
\hline Absorption correction & $\begin{array}{l}\text { Semi-empirical from } \\
\text { equivalents }\end{array}$ & $\begin{array}{l}\text { Semi-empirical from } \\
\text { equivalents }\end{array}$ & $\begin{array}{l}\text { Semi-empirical from } \\
\text { equivalents }\end{array}$ & $\begin{array}{l}\text { Semi-empirical from } \\
\text { equivalents }\end{array}$ \\
\hline Max. and min. transmission & 1.00000 and 0.82297 & 1.00000 and 0.83660 & 1.00000 and 0.47517 & 1.00000 and 0.48302 \\
\hline Refinement method & Full-matrix least-squares on $\mathrm{F}^{2}$ & Full-matrix least-squares on $\mathrm{F}^{2}$ & Full-matrix least-squares on $\mathrm{F}^{2}$ & Full-matrix least-squares on $\mathrm{F}^{2}$ \\
\hline Data / restraints / parameters & $7109 / 11 / 721$ & 4897 / 43 / 397 & $8021 / 2 / 478$ & $14578 / 310 / 938$ \\
\hline Goodness-of-fit on $\mathrm{F}^{2}$ & 1.026 & 1.046 & 1.289 & 1.060 \\
\hline Final $\mathrm{R}$ indices $[\mathrm{I}>2 \operatorname{sigma}(\mathrm{I})]$ & $\mathrm{R} 1=0.0568, \mathrm{wR} 2=0.1430$ & $\mathrm{R} 1=0.0626, \mathrm{wR} 2=0.1659$ & $\mathrm{R} 1=0.1329, \mathrm{wR} 2=0.3681$ & $\mathrm{R} 1=0.1479, \mathrm{wR} 2=0.4041$ \\
\hline $\mathrm{R}$ indices (all data) & $\mathrm{R} 1=0.0743, \mathrm{wR} 2=0.1555$ & $\mathrm{R} 1=0.0877, \mathrm{wR} 2=0.1845$ & $\mathrm{R} 1=0.2051, \mathrm{wR} 2=0.4214$ & $\mathrm{R} 1=0.1649, \mathrm{wR} 2=0.4154$ \\
\hline Extinction coefficient & $\mathrm{n} / \mathrm{a}$ & $\mathrm{n} / \mathrm{a}$ & $\mathrm{n} / \mathrm{a}$ & $\mathrm{n} / \mathrm{a}$ \\
\hline Largest diff. peak and hole & 0.305 and $-0.210 \mathrm{e} . \AA^{-3}$ & 0.595 and $-0.334 \mathrm{e} . \AA^{-3}$ & 0.960 and -0.726 e. $\AA^{-3}$ & 1.249 and $-0.593 \mathrm{e} . \AA^{-3}$ \\
\hline
\end{tabular}




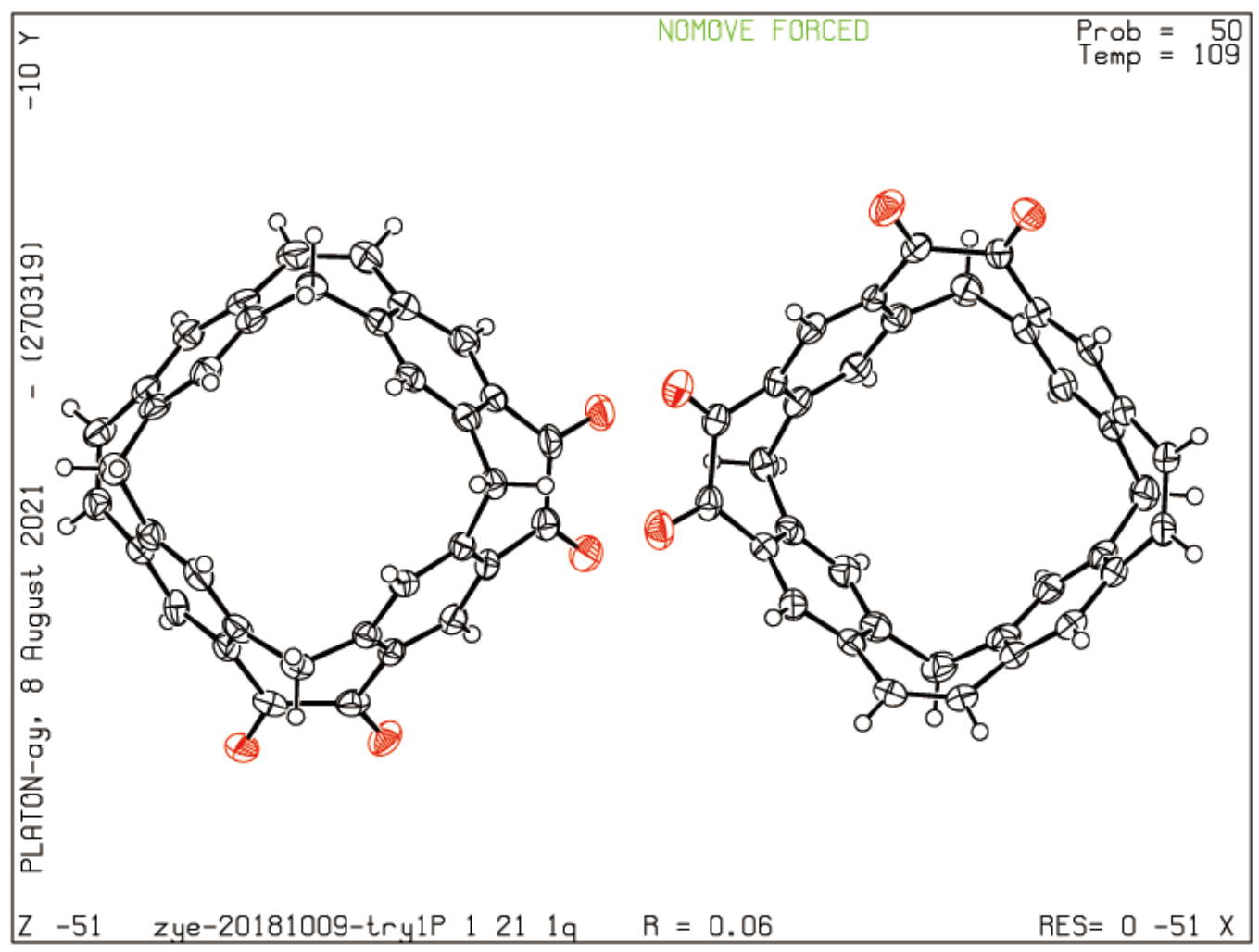

Figure S1. X-ray molecular structure of $\mathbf{3}$ (CCDC 2102075). The molecular is depicted in stick-ellipsoid style at $50 \%$ probability level for all atoms.

Alert level B

PLAT915_ALERT_3_B No Flack x Check Done: Low Friedel Pair Coverage

Explain: This alert is caused by molecules themselves because they are achiral. 


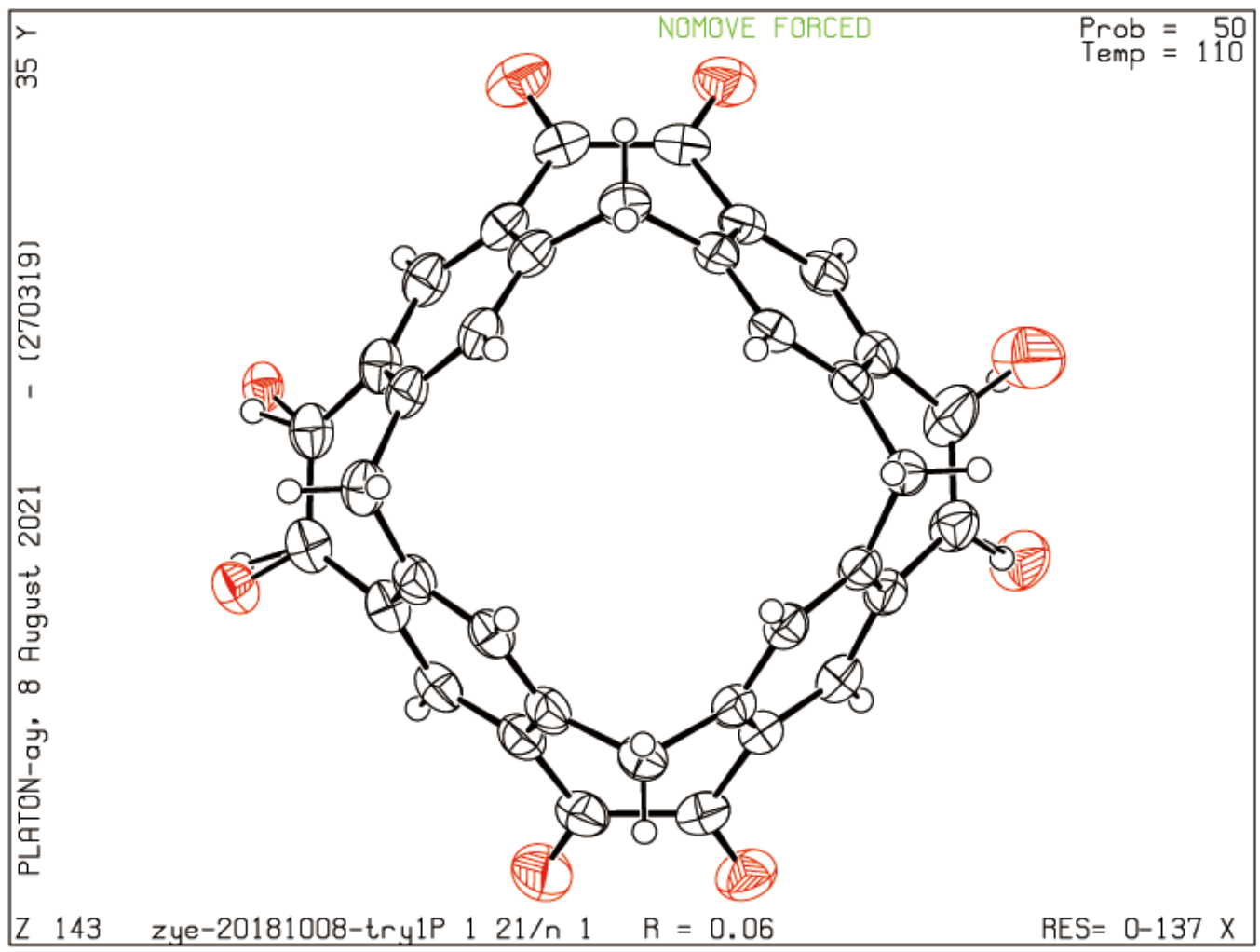

Figure S2. X-ray molecular structure of $\mathbf{5}$ (CCDC 2102076). The molecular is depicted in stick-ellipsoid style at $50 \%$ probability level for all atoms. 


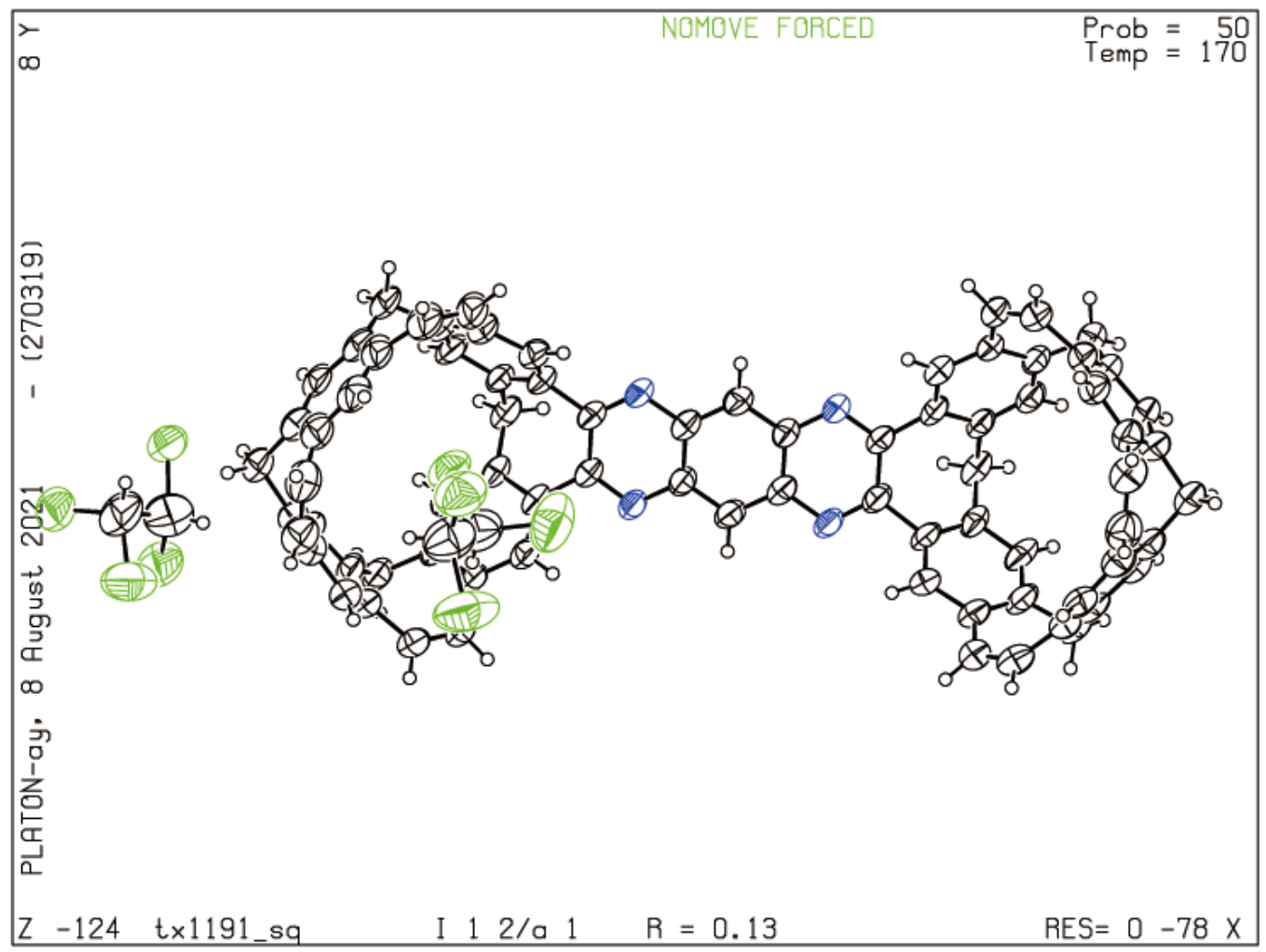

Figure S3. X-ray molecular structure of 14 (CCDC 2102077). The molecular is depicted in stick-ellipsoid style at $50 \%$ probability level for all atoms.

Alert level B

PLAT084_ALERT_3_B No High wR2 Value (i.e. > 0.25)................0.42 Report PLAT340_ALERT_3_B Lower Bond Precision on C-C Bonds........... 0.01143 Ang.

Explain: This alert is caused by poor crystal quality. 


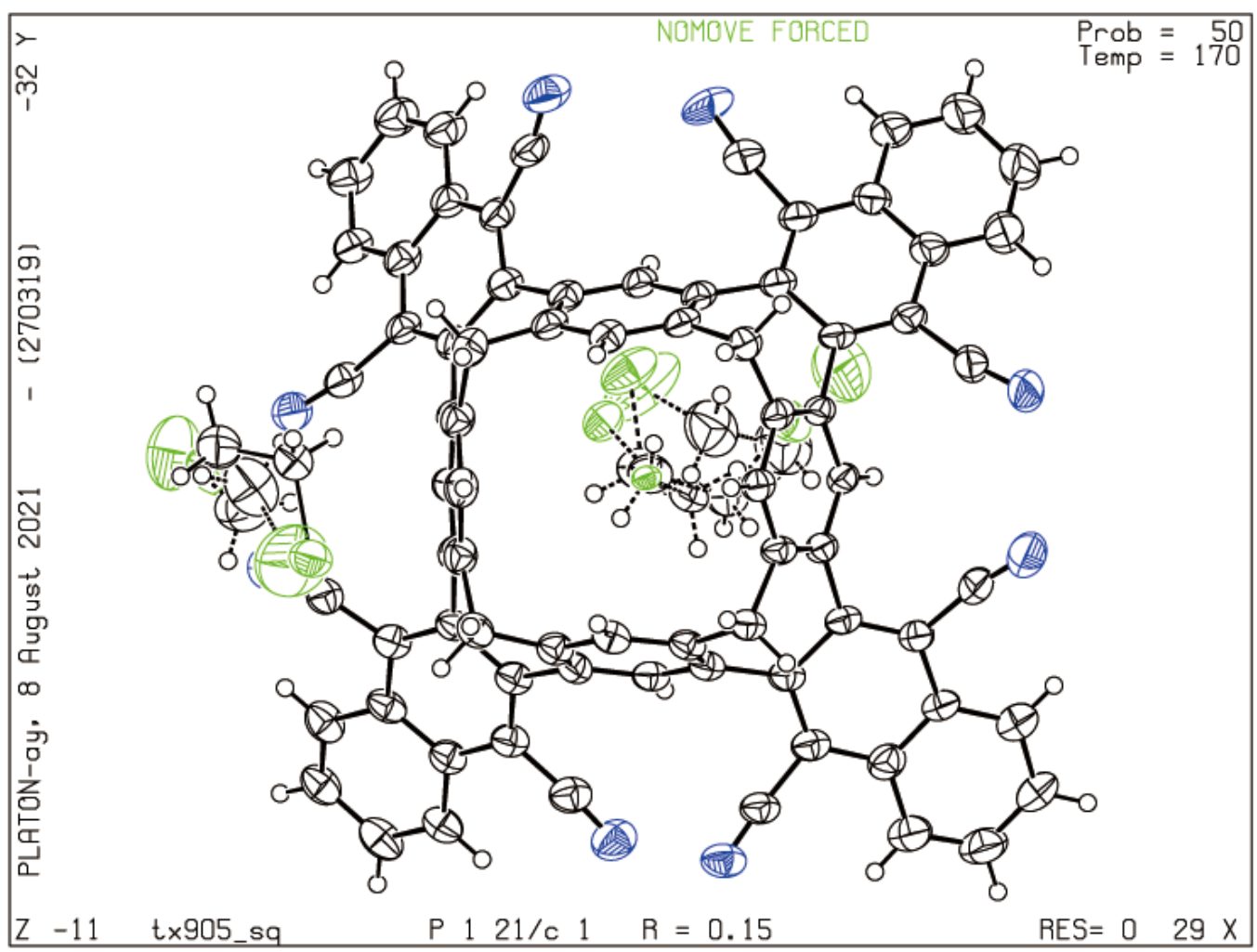

Figure S4. X-ray molecular structure of 19 (CCDC 2102078). The molecular is depicted in stick-ellipsoid style at 50\% probability level for all atoms.

Alert level B

PLAT084_ALERT_3_B No High wR2 Value (i.e. > 0.25). 0.42 Report

Explain: This alert is caused by poor crystal quality.

\section{Refrences}

1. Q. Zhang, Y.-E. Zhang, S. Tong, M.-X. Wang, J. Am. Chem. Soc. 2020, 142, 11961199.

2. T. Nishiuchi, M. Iyoda, Bull. Chem. Soc. Jpn. 2014, 87, 960-973. 
5. Summary of photophysical properties of $9,10,15,16,19$ and 20

\begin{tabular}{ccc}
\hline Comp. & $\lambda_{\text {abs }}(\mathrm{nm}) / \varepsilon\left(\mathrm{L} \cdot \mathrm{mol}^{-1} \cdot \mathrm{cm}^{-1}\right)^{[\mathrm{a}]}$ & $\lambda_{\mathrm{em}}(\mathrm{nm}) / \Phi_{\mathrm{f}}(\%)$ \\
\hline $\mathbf{9}$ & $243 / 2929 ; 262 / 3283 ; 370 / 440$ & $538 / 1.1^{[\mathrm{b}]}$ \\
$\mathbf{1 0}$ & $249 / 3881 ; 269 / 2859 ; 350 / 459$ & $494 / 1.5^{[\mathrm{c}]}$ \\
$\mathbf{1 5}$ & $264 / 3833 ; 281 / 3099 ; 367 / 1001$ & $524 / 1.9^{[\mathrm{b}]}$ \\
$\mathbf{1 6}$ & $249 / 3939 ; 270 / 4519 ; 345 / 1345$ & $487 / 2.2^{[\mathrm{c}]}$ \\
$\mathbf{1 9}$ & $257 / 3083 ; 281 / 2550 ; 365 / 833$ & $407 / 40.7^{[\mathrm{b}]}$ \\
$\mathbf{2 0}$ & $249 / 4763 ; 265 / 5050 ; 345 / 1910$ & $408 / 0.3^{[\mathrm{c}]}$ \\
\hline
\end{tabular}

[a] $9\left(\mathrm{c}=2.5 \times 10^{-4} \mathrm{~mol} / \mathrm{L}\right), 10\left(\mathrm{c}=2.0 \times 10^{-4} \mathrm{~mol} / \mathrm{L}\right), 15\left(\mathrm{c}=2.0 \times 10^{-4} \mathrm{~mol} / \mathrm{L}\right), 16(\mathrm{c}=$ $\left.2.0 \times 10^{-4} \mathrm{~mol} / \mathrm{L}\right), \mathbf{1 9}\left(\mathrm{c}=2.5 \times 10^{-4} \mathrm{~mol} / \mathrm{L}\right), \mathbf{2 0}\left(\mathrm{c}=1.0 \times 10^{-4} \mathrm{~mol} / \mathrm{L}\right)$. [b] Using quinine [b] Sulfate in $0.1 \mathrm{M} \mathrm{H}_{2} \mathrm{SO}_{4}$ as refrence, $\Phi_{\mathrm{f}}=0.54, \lambda_{\mathrm{ex}}=360 \mathrm{~nm}$. [c] Using quinine Sulfate in $0.1 \mathrm{M} \mathrm{H}_{2} \mathrm{SO}_{4}$ as refrence, $\Phi_{\mathrm{f}}=0.54, \lambda_{\mathrm{ex}}=350 \mathrm{~nm}$. 


\section{UV/Vis and Fluorescence Spectra of 9, 10, 15, 16, 19, 20}
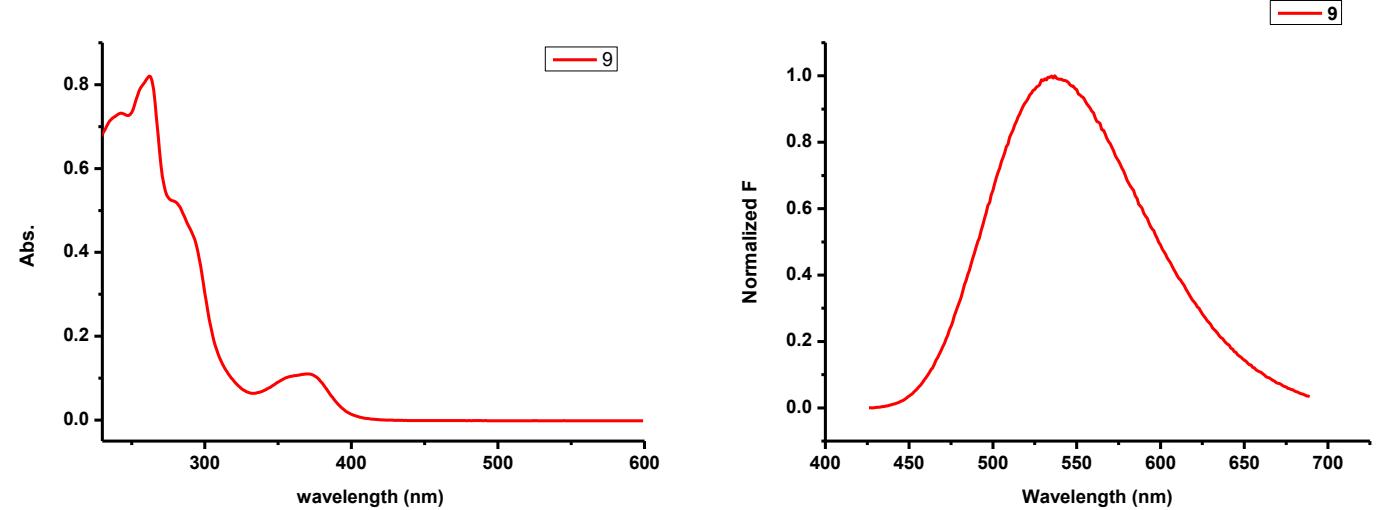

The UV-Vis spectrum of $9\left(c=2.5 \times 10^{-4} \mathrm{~mol} / \mathrm{L}\right)$ in dichlorometane at $293 \mathrm{~K}$ (left) and the normalized fluorescence spectrum of 9 in dichlorometane at $293 \mathrm{~K}$ (right). Fluorescence spectrum was measured with excitation wavelength of $360 \mathrm{~nm}$, and using Em. slit width of $10 \mathrm{~nm}$ and Ex. slit width of $5 \mathrm{~nm}$.
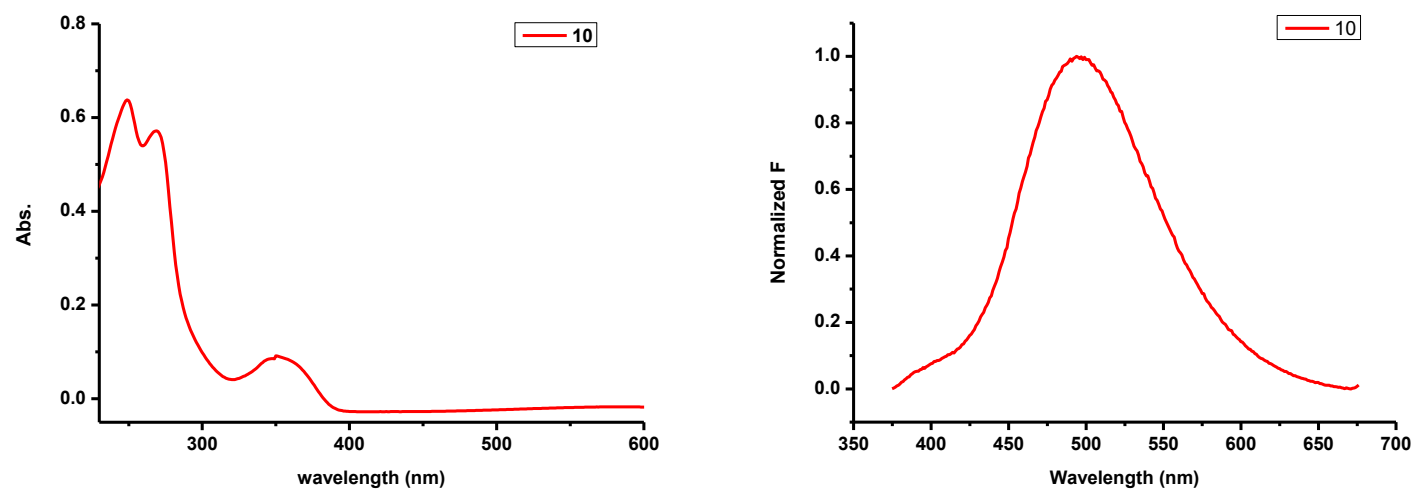

The UV-Vis spectrum of $\mathbf{1 0}\left(c=2.0 \times 10^{-4} \mathrm{~mol} / \mathrm{L}\right)$ in dichlorometane at $293 \mathrm{~K}$ (left) and the normalized fluorescence spectrum of $\mathbf{1 0}$ in dichlorometane at $293 \mathrm{~K}$ (right). Fluorescence spectrum was measured with excitation wavelength of $345 \mathrm{~nm}$, and using Em. slit width of $10 \mathrm{~nm}$ and Ex. slit width of $5 \mathrm{~nm}$.
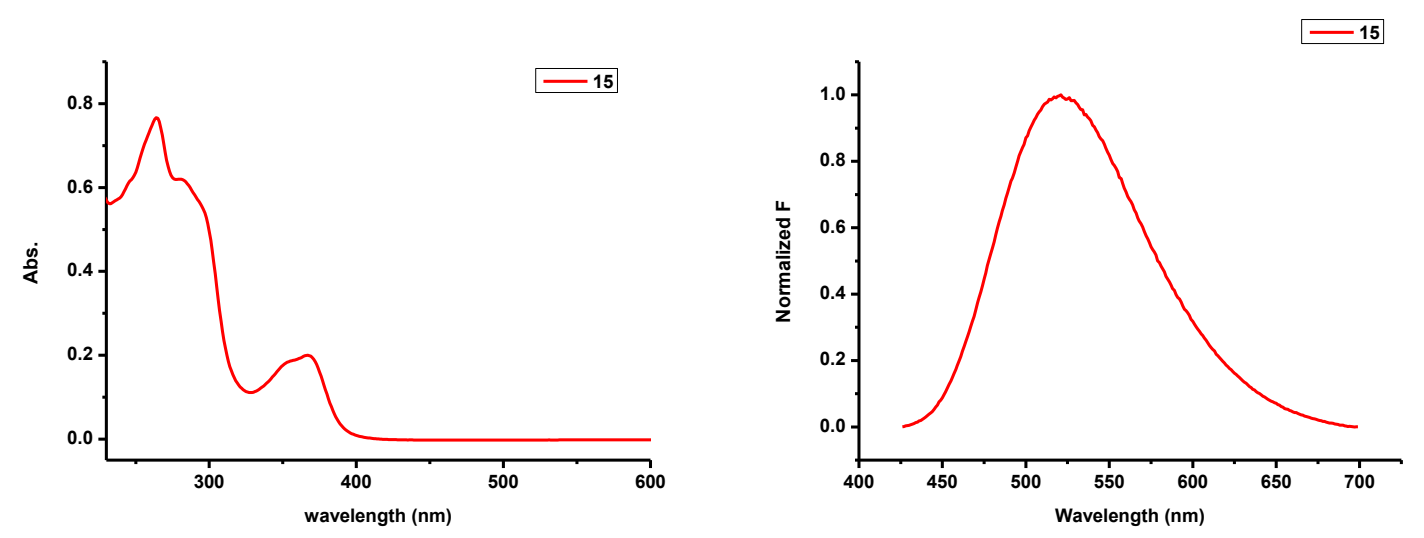

The UV-Vis spectrum of $\mathbf{1 5}\left(c=2.0 \times 10^{-4} \mathrm{~mol} / \mathrm{L}\right)$ in dichlorometane at $293 \mathrm{~K}$ (left) and the 
normalized fluorescence spectrum of $\mathbf{1 5}$ in dichlorometane at $293 \mathrm{~K}$ (right). Fluorescence spectrum was measured with excitation wavelength of $360 \mathrm{~nm}$, and using Em. slit width of $10 \mathrm{~nm}$ and Ex. slit width of $5 \mathrm{~nm}$.
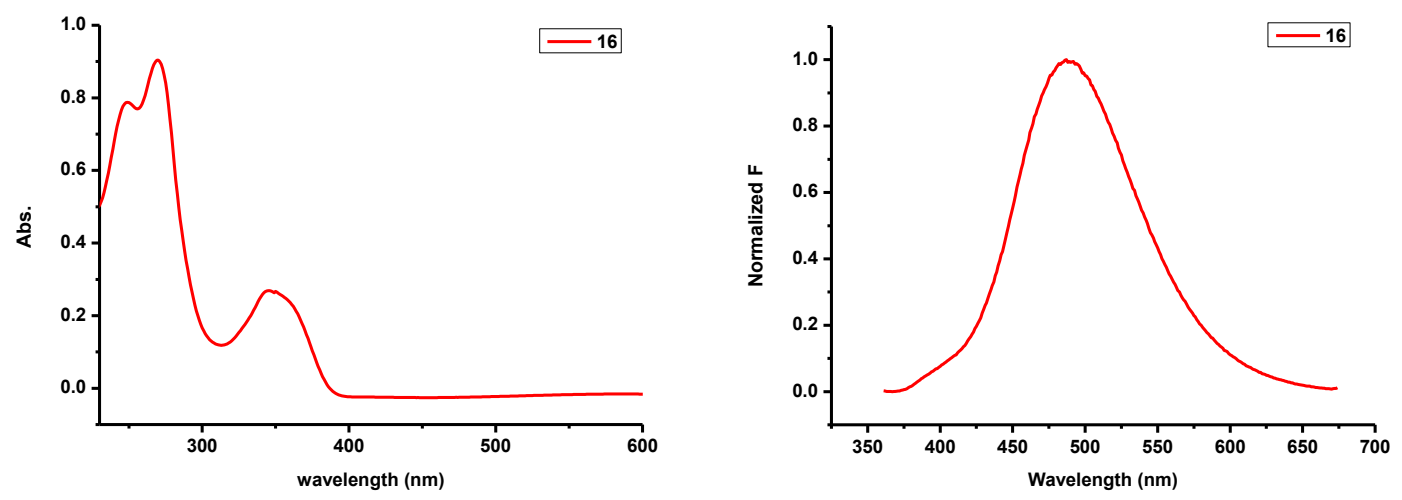

The UV-Vis spectrum of $\mathbf{1 6}\left(c=2.0 \times 10^{-4} \mathrm{~mol} / \mathrm{L}\right)$ in dichlorometane at $293 \mathrm{~K}$ (left) and the normalized fluorescence spectrum of $\mathbf{1 6}$ in dichlorometane at $293 \mathrm{~K}$ (right). Fluorescence spectrum was measured with excitation wavelength of $345 \mathrm{~nm}$, and using Em. slit width of $10 \mathrm{~nm}$ and Ex. slit width of $5 \mathrm{~nm}$.
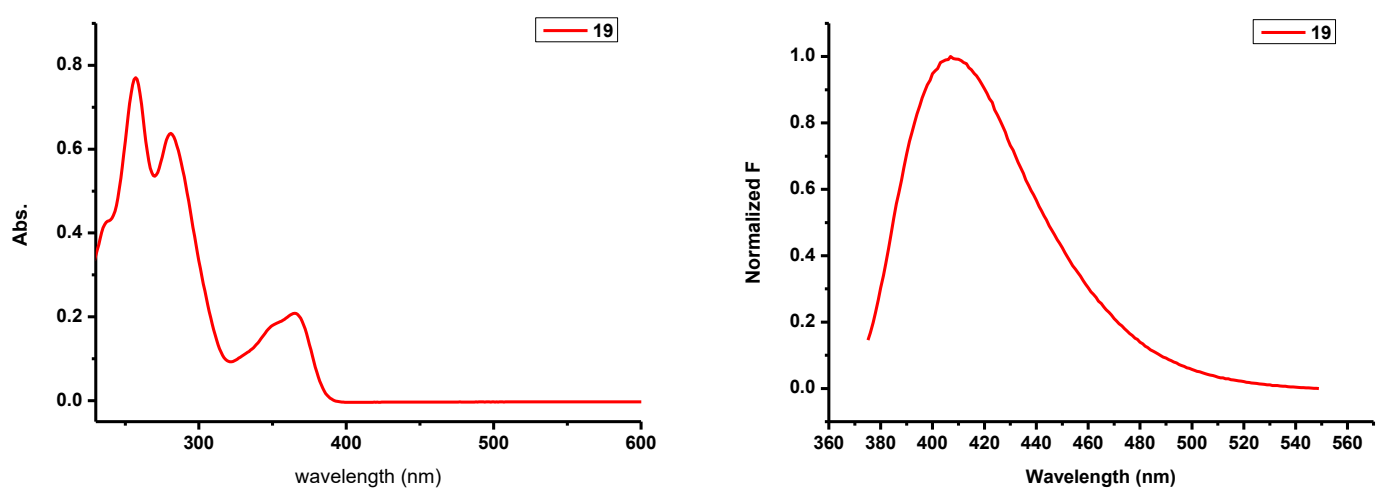

The UV-Vis spectrum of $\mathbf{1 9}\left(c=1.5 \times 10^{-4} \mathrm{~mol} / \mathrm{L}\right)$ in dichlorometane at $293 \mathrm{~K}$ (left) and the normalized fluorescence spectrum of 19 in dichlorometane at $293 \mathrm{~K}$ (right). Fluorescence spectrum was measured with excitation wavelength of $360 \mathrm{~nm}$, and using Em. slit width of $10 \mathrm{~nm}$ and Ex. slit width of $5 \mathrm{~nm}$. 

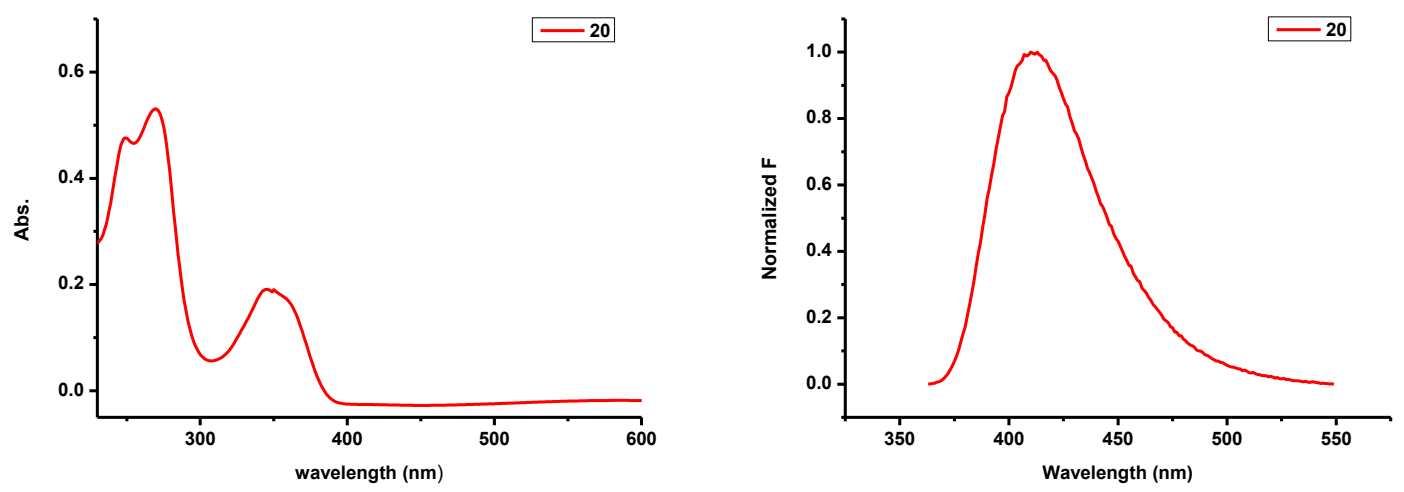

The UV-Vis spectrum of $\mathbf{2 0}\left(c=1.5 \times 10^{-4} \mathrm{~mol} / \mathrm{L}\right)$ in dichlorometane at $293 \mathrm{~K}$ (left) and the normalized fluorescence spectrum of $\mathbf{2 0}$ in dichlorometane at $293 \mathrm{~K}$ (right). Fluorescence spectrum was measured with excitation wavelength of $345 \mathrm{~nm}$, and using Em. slit width of $10 \mathrm{~nm}$ and Ex. slit width of $5 \mathrm{~nm}$.

\section{7. ${ }^{1} \mathrm{H}$ and ${ }^{13} \mathrm{C}$ NMR Spectra of products}


${ }^{1} \mathrm{H}$ NMR for $2(400 \mathrm{MHz})$

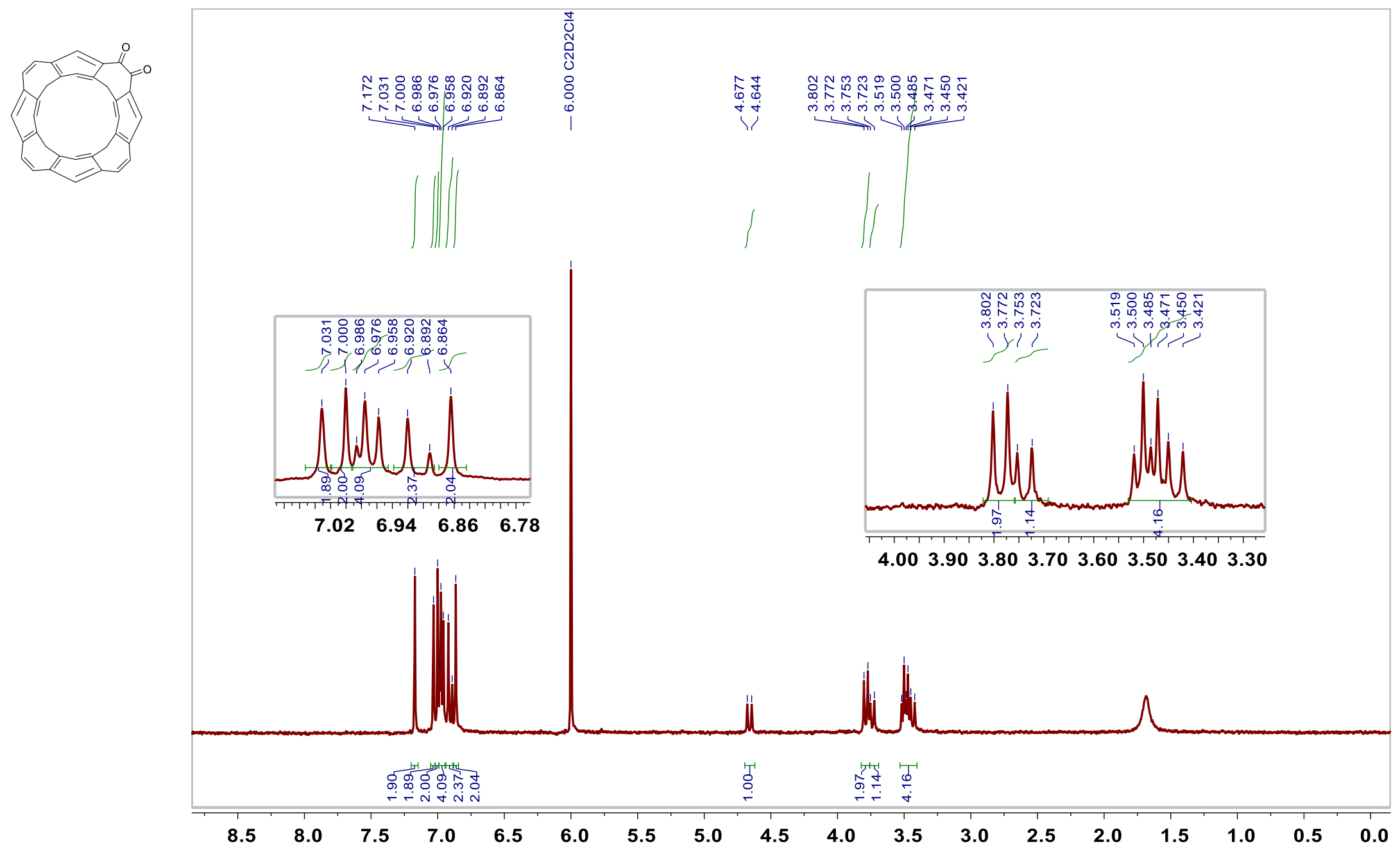


${ }^{13} \mathrm{C}$ NMR for $2(100 \mathrm{MHz})$

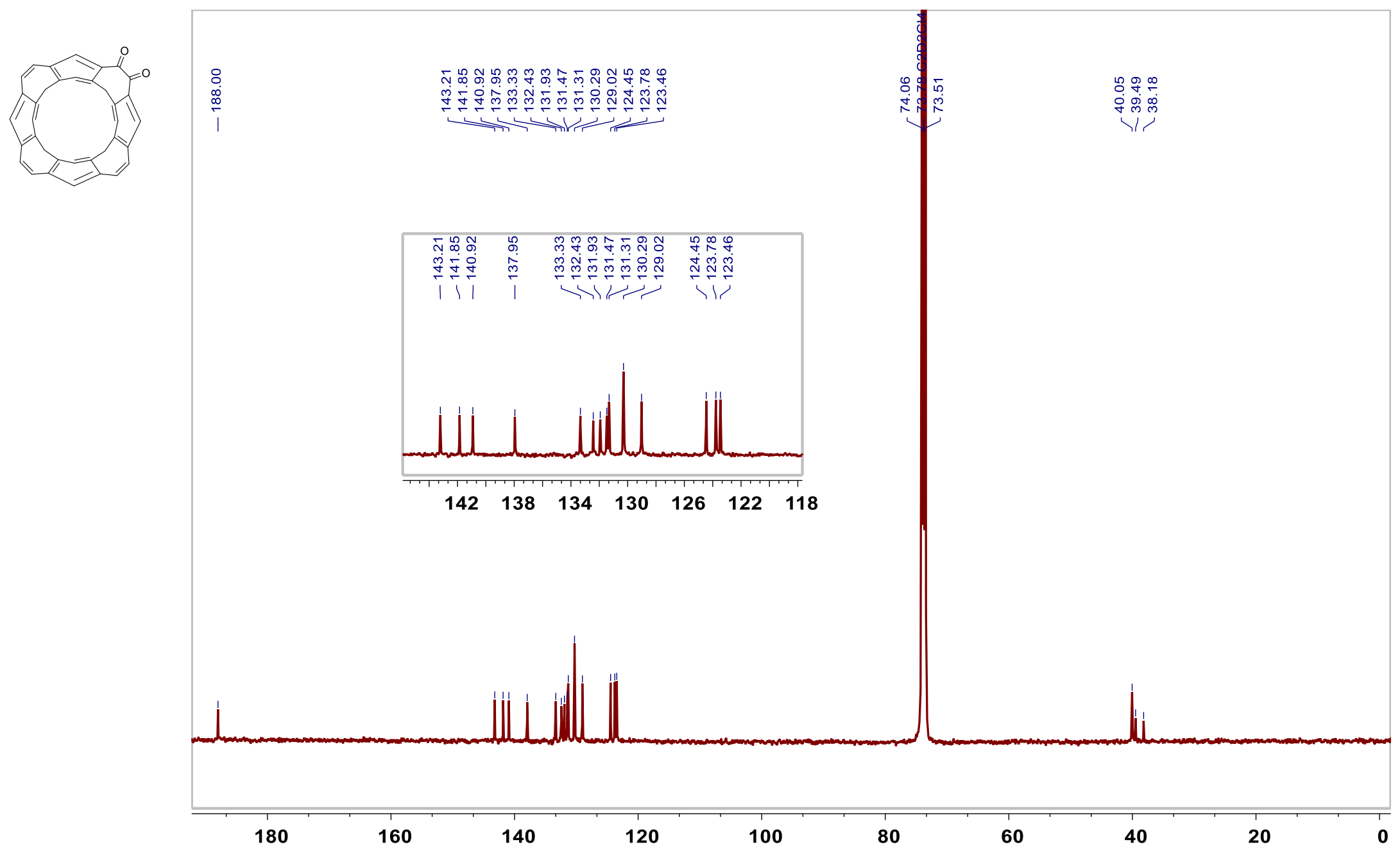


${ }^{1} \mathrm{H}$ NMR for 3 (400 MHz)

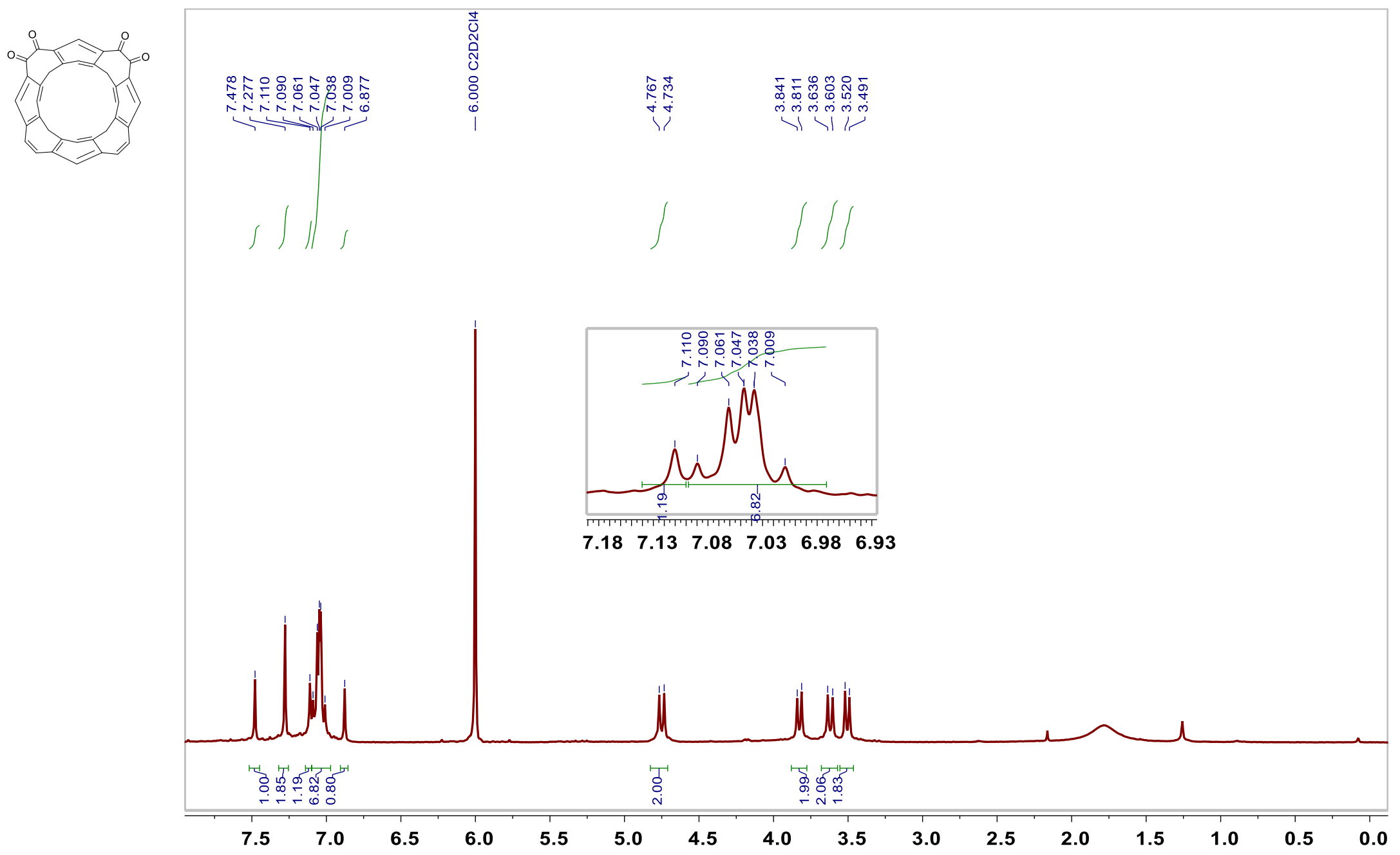


${ }^{13} \mathrm{C}$ NMR for 3 (100 MHz)

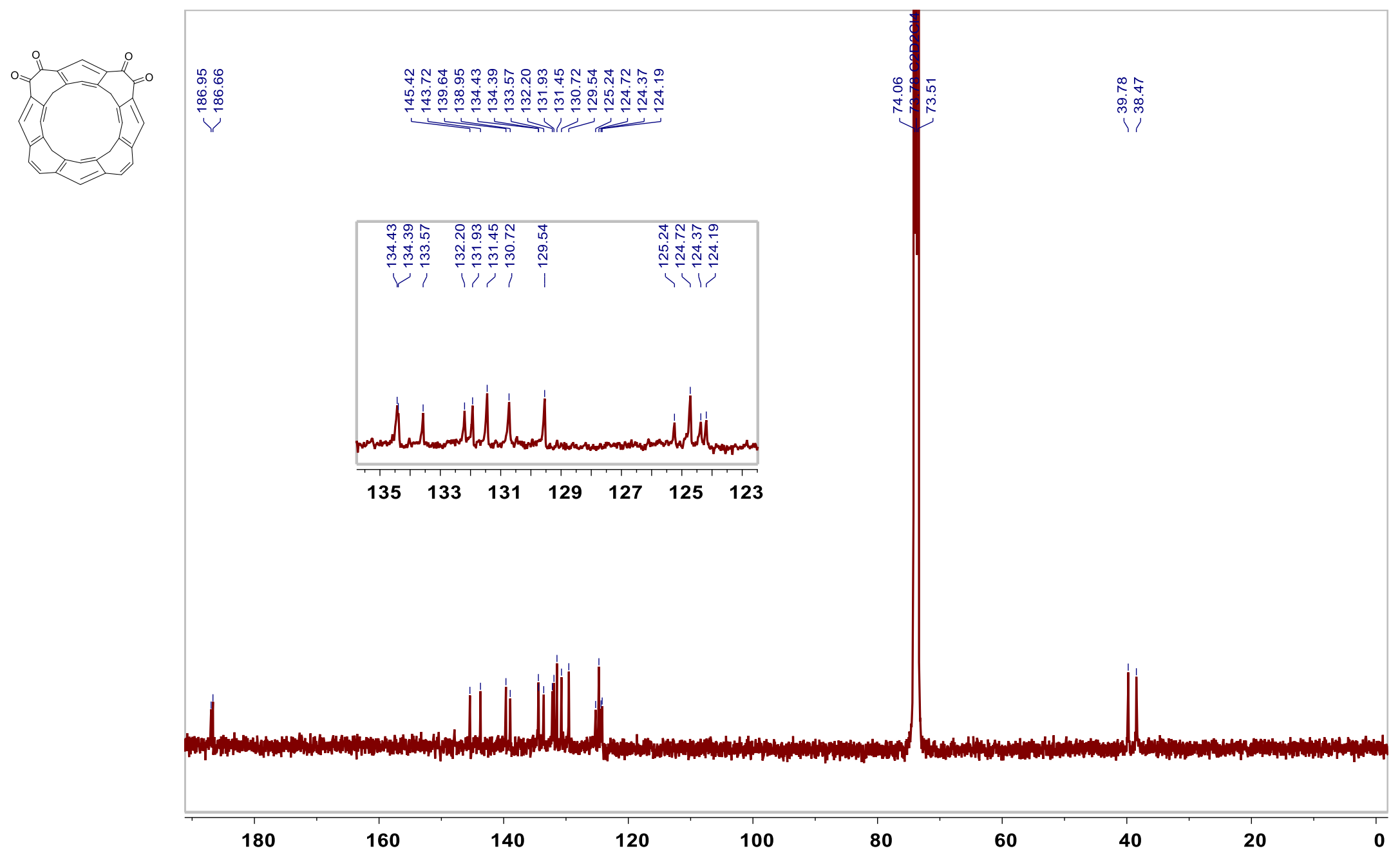




\section{${ }^{1} \mathrm{H}$ NMR for 4 (400 MHz)}

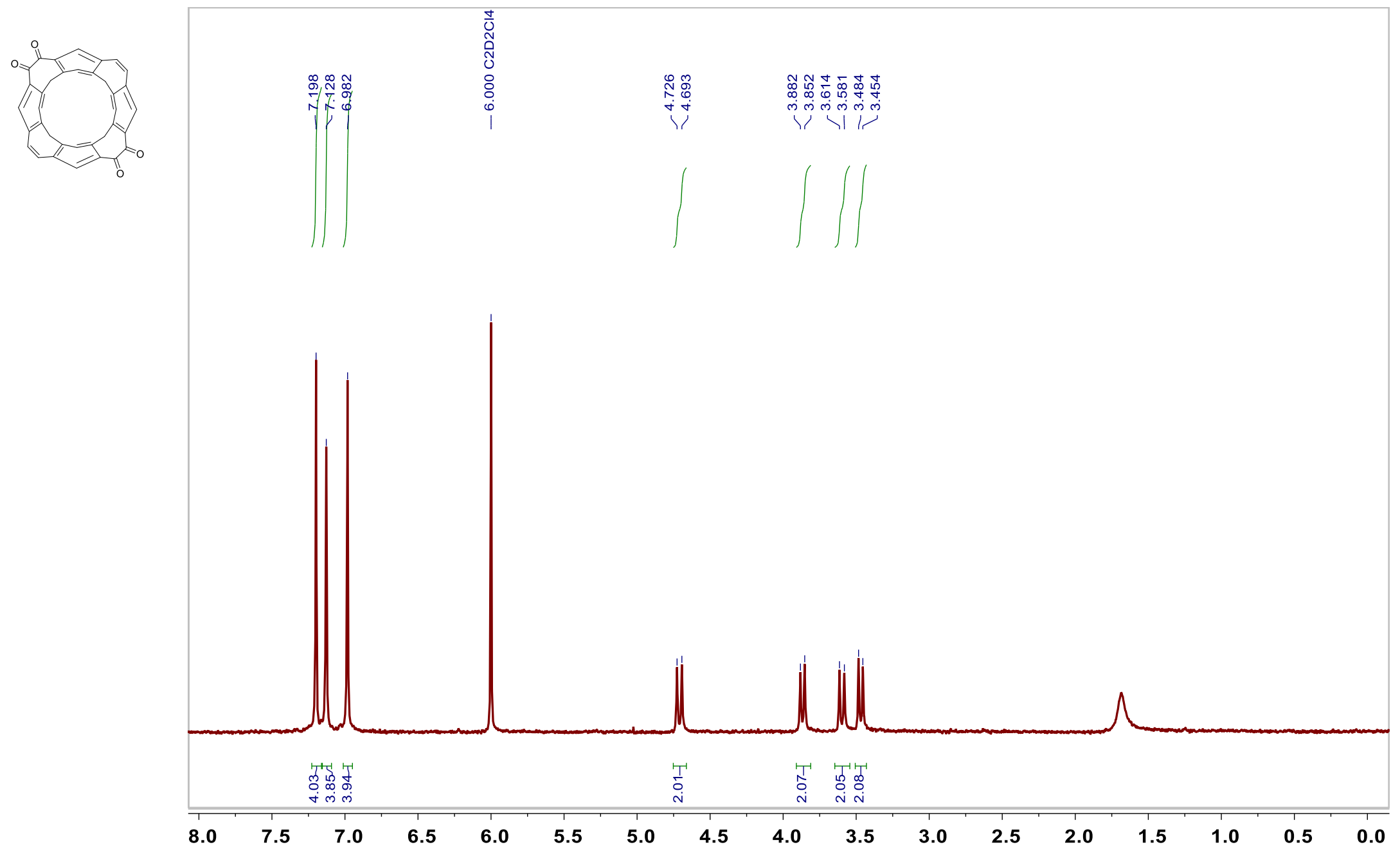


${ }^{13} \mathrm{C}$ NMR for 4 (100 MHz)

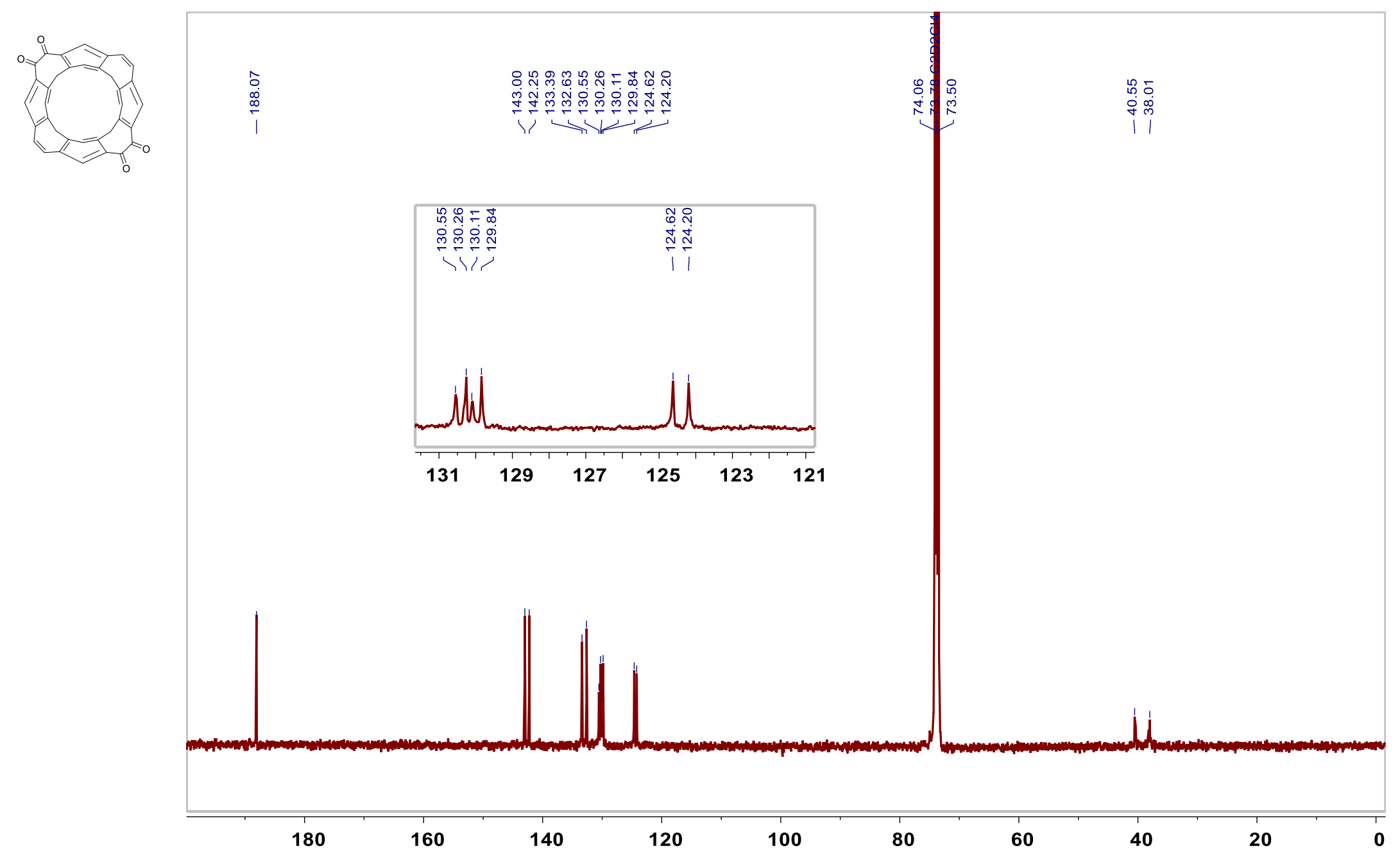


${ }^{1} \mathrm{H}$ NMR for 5 (400 MHz)

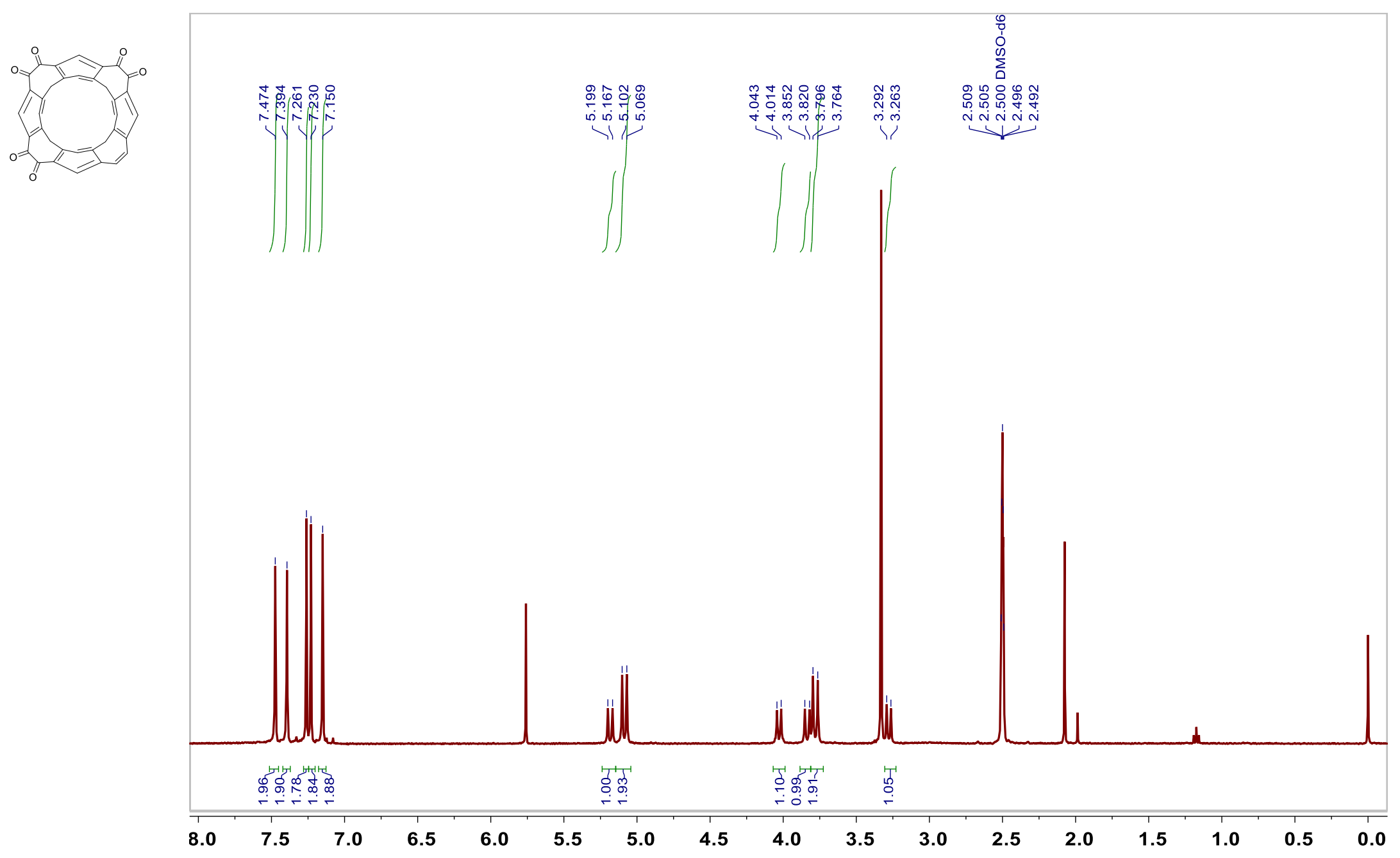

S30 
${ }^{13} \mathrm{C}$ NMR for 5 (100 MHz)

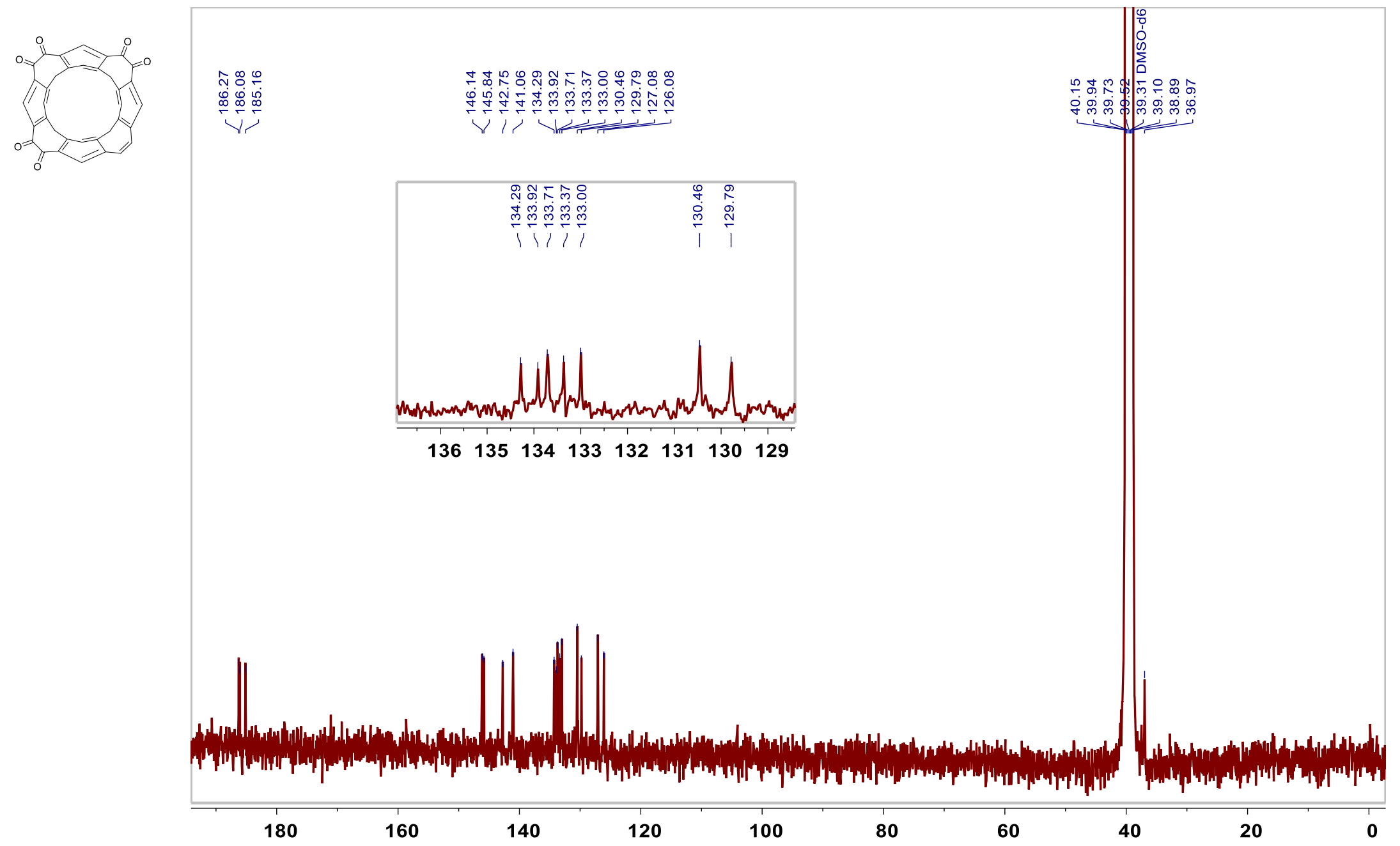


${ }^{1} \mathrm{H}$ NMR for 6 (400 MHz)

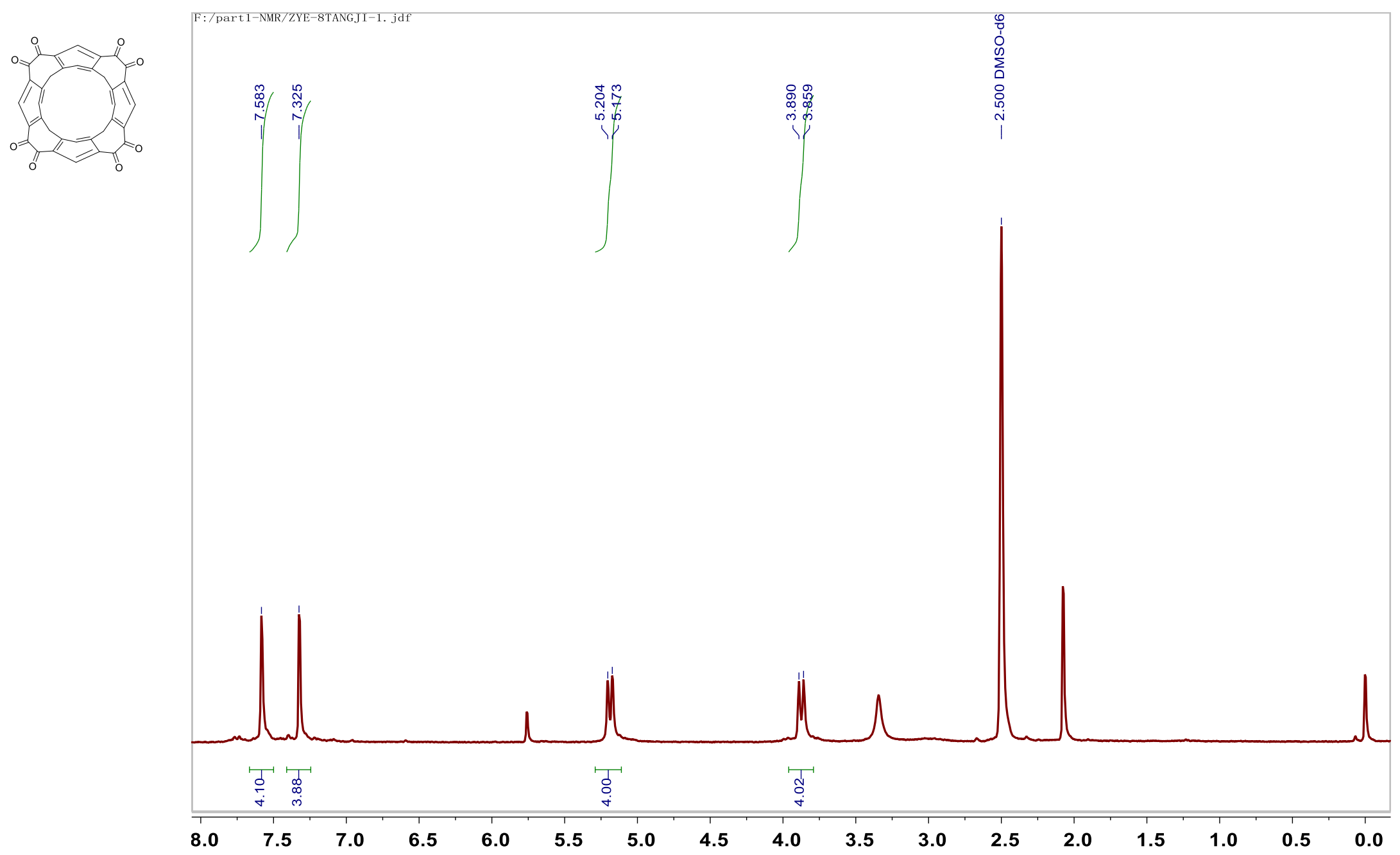


${ }^{13} \mathrm{C}$ NMR for $6(100 \mathrm{MHz})$

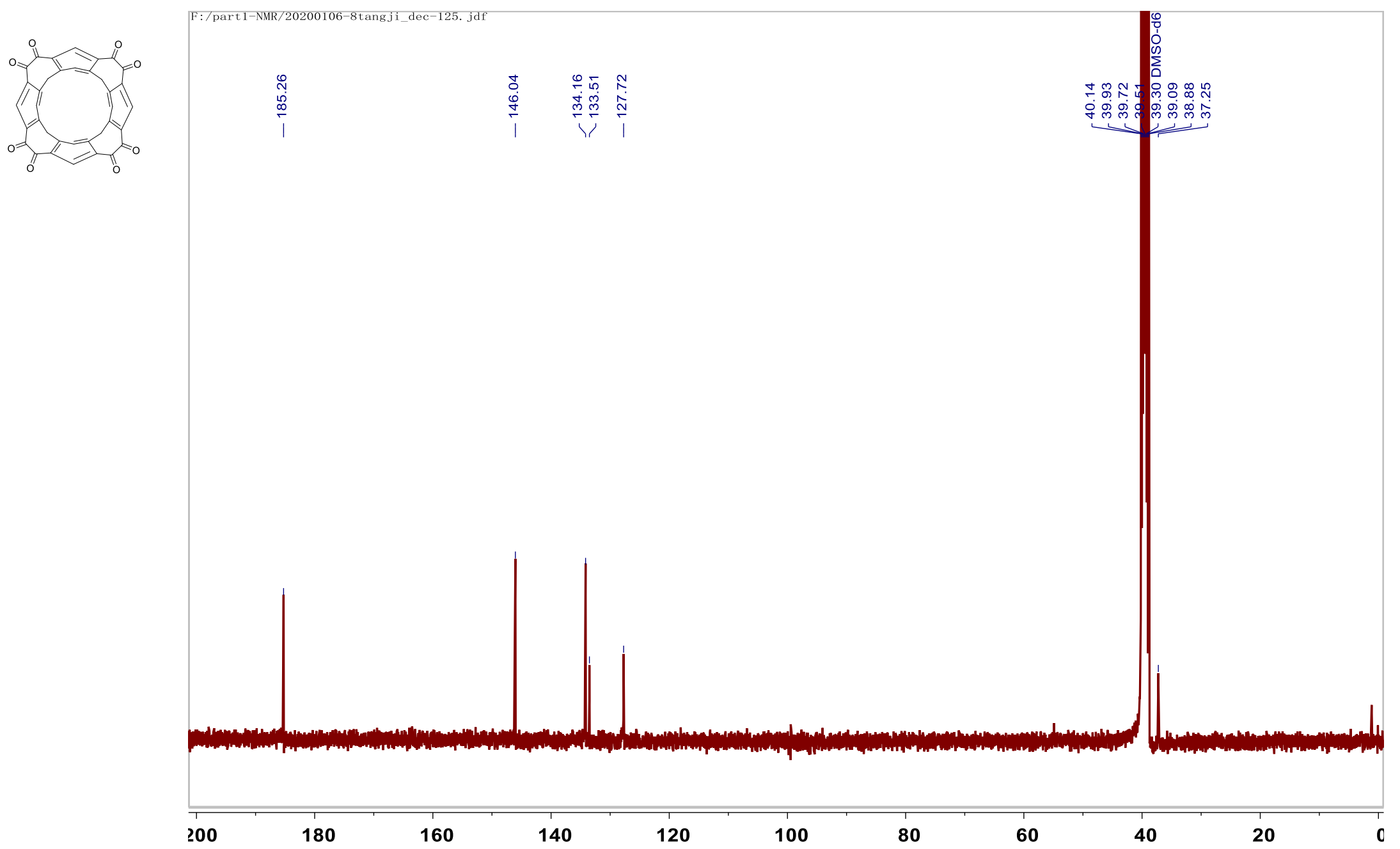


${ }^{1} \mathrm{H}$ NMR for 9 (400 MHz)

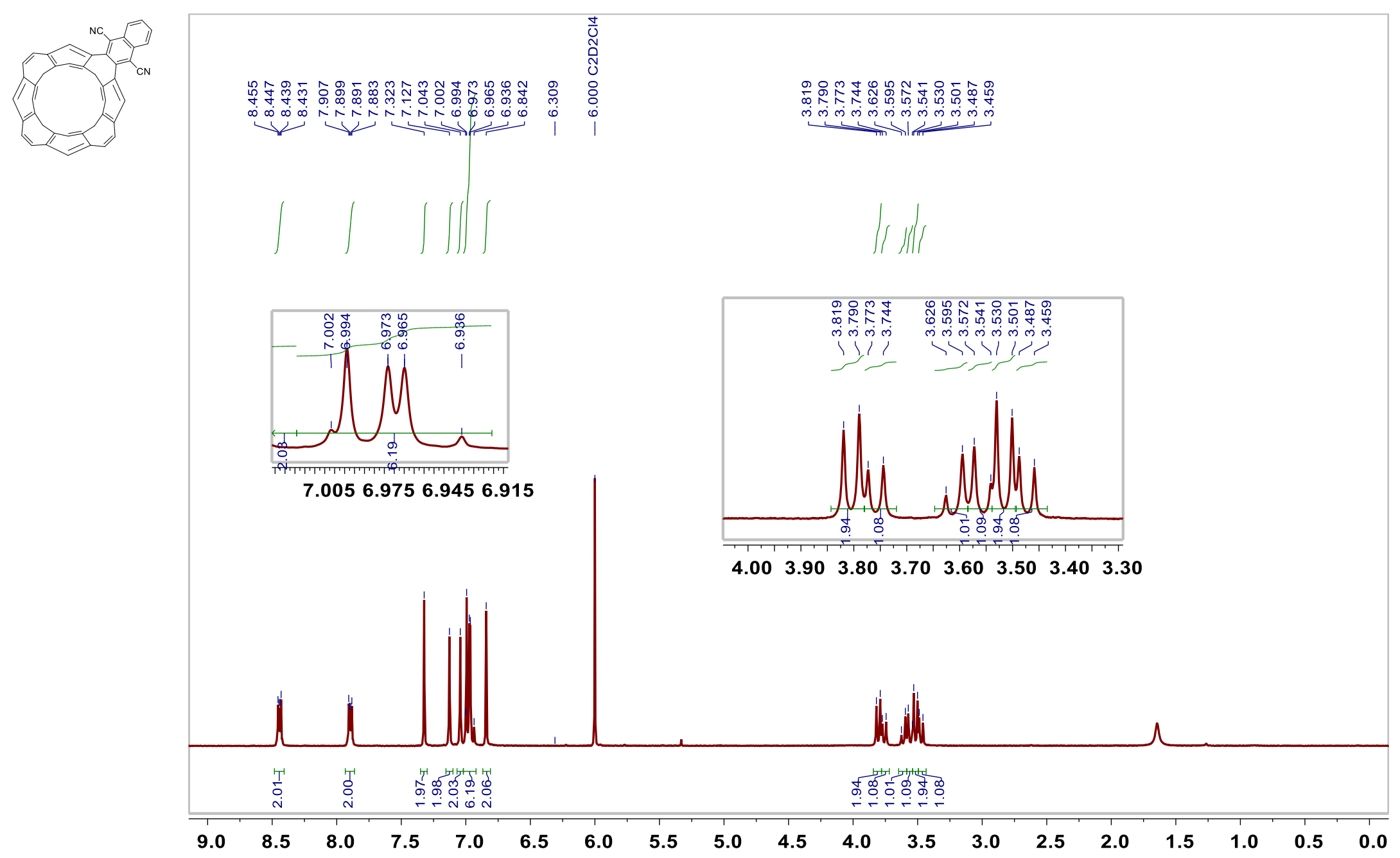


${ }^{13} \mathrm{C}$ NMR for $9(100 \mathrm{MHz})$

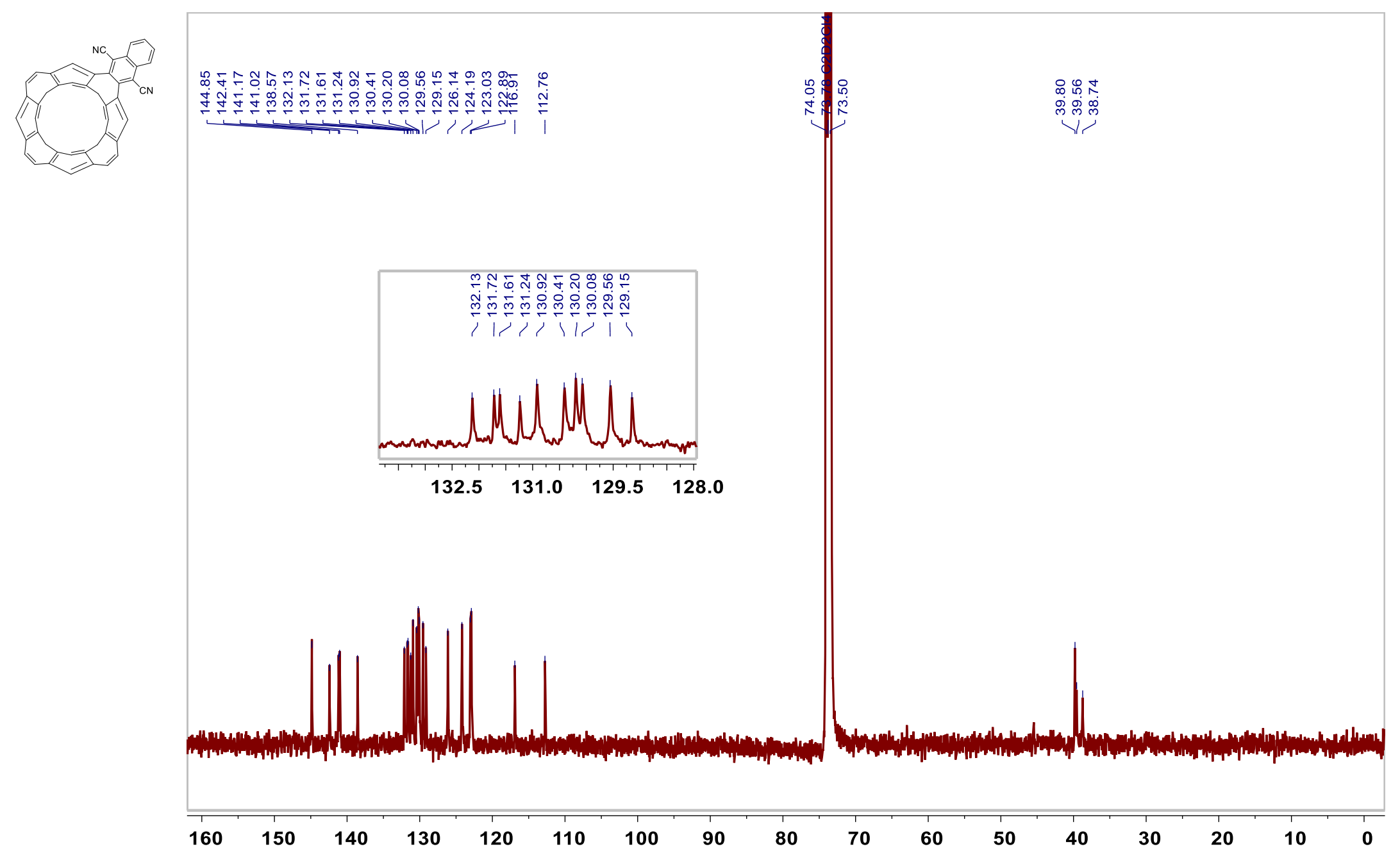


${ }^{1} \mathrm{H}$ NMR for $10(400 \mathrm{MHz})$

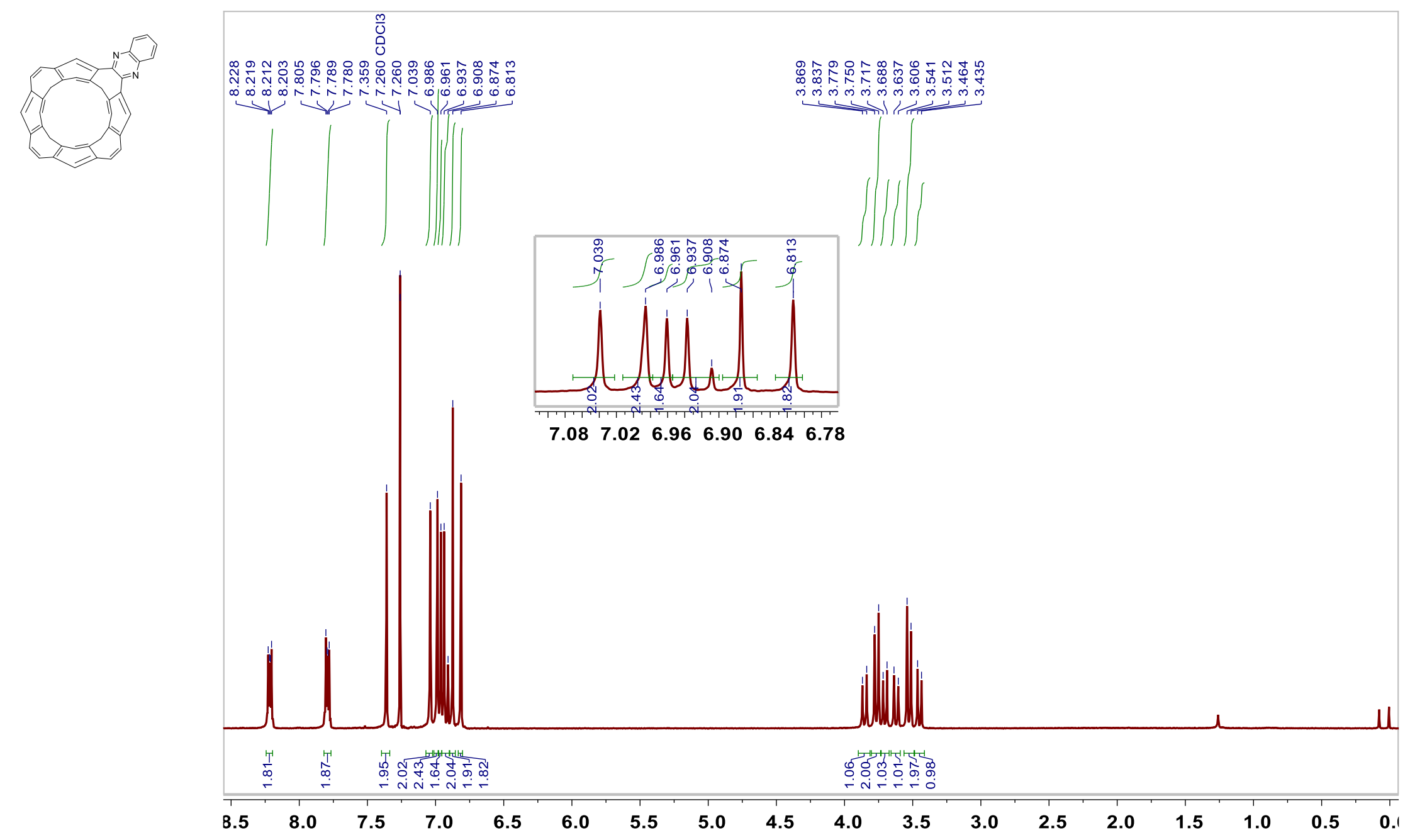


${ }^{13} \mathrm{C}$ NMR for $10(100 \mathrm{MHz})$

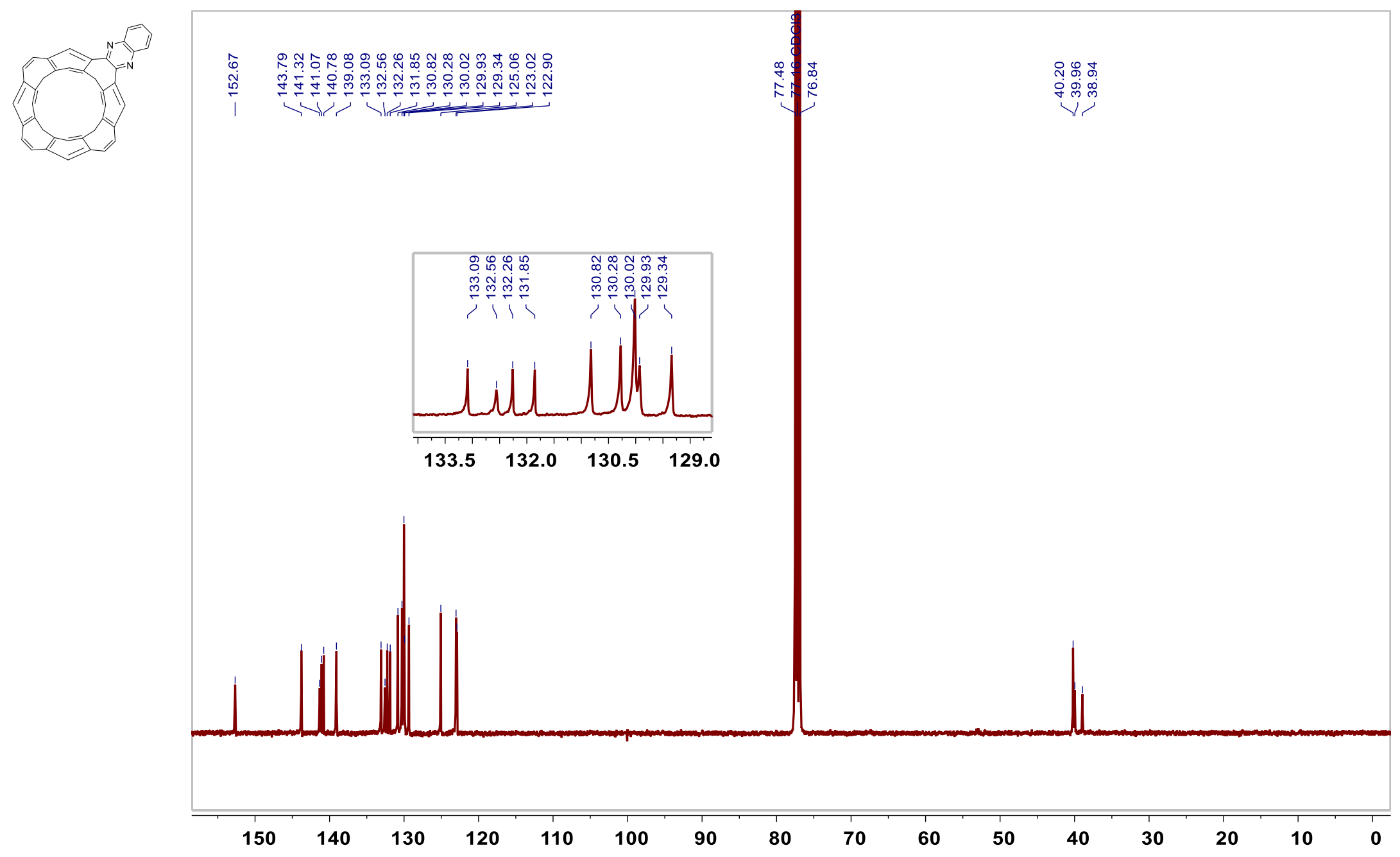


${ }^{1} \mathrm{H}$ NMR for 13 (400 MHz)

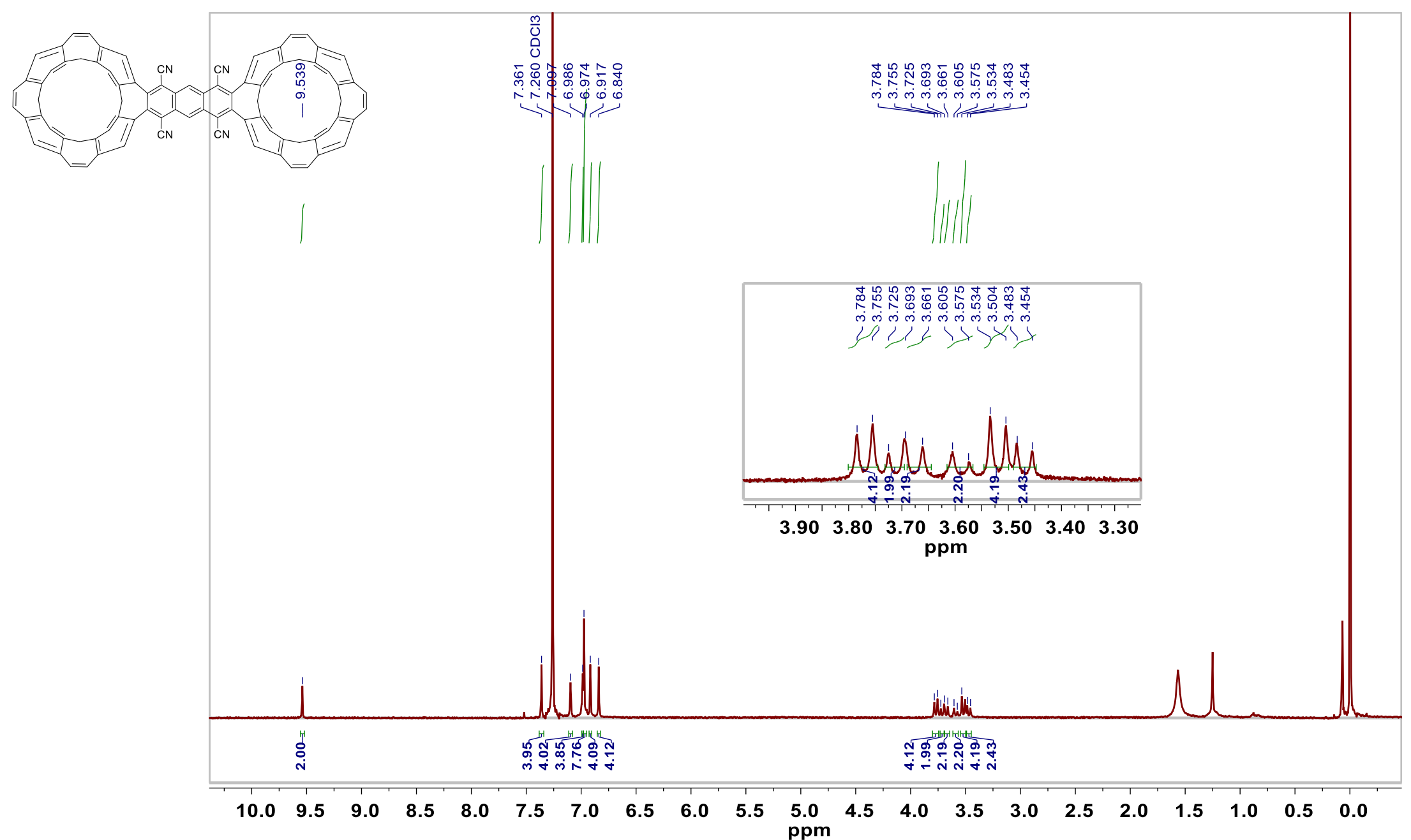


${ }^{13} \mathrm{C}$ NMR for $13(100 \mathrm{MHz})$

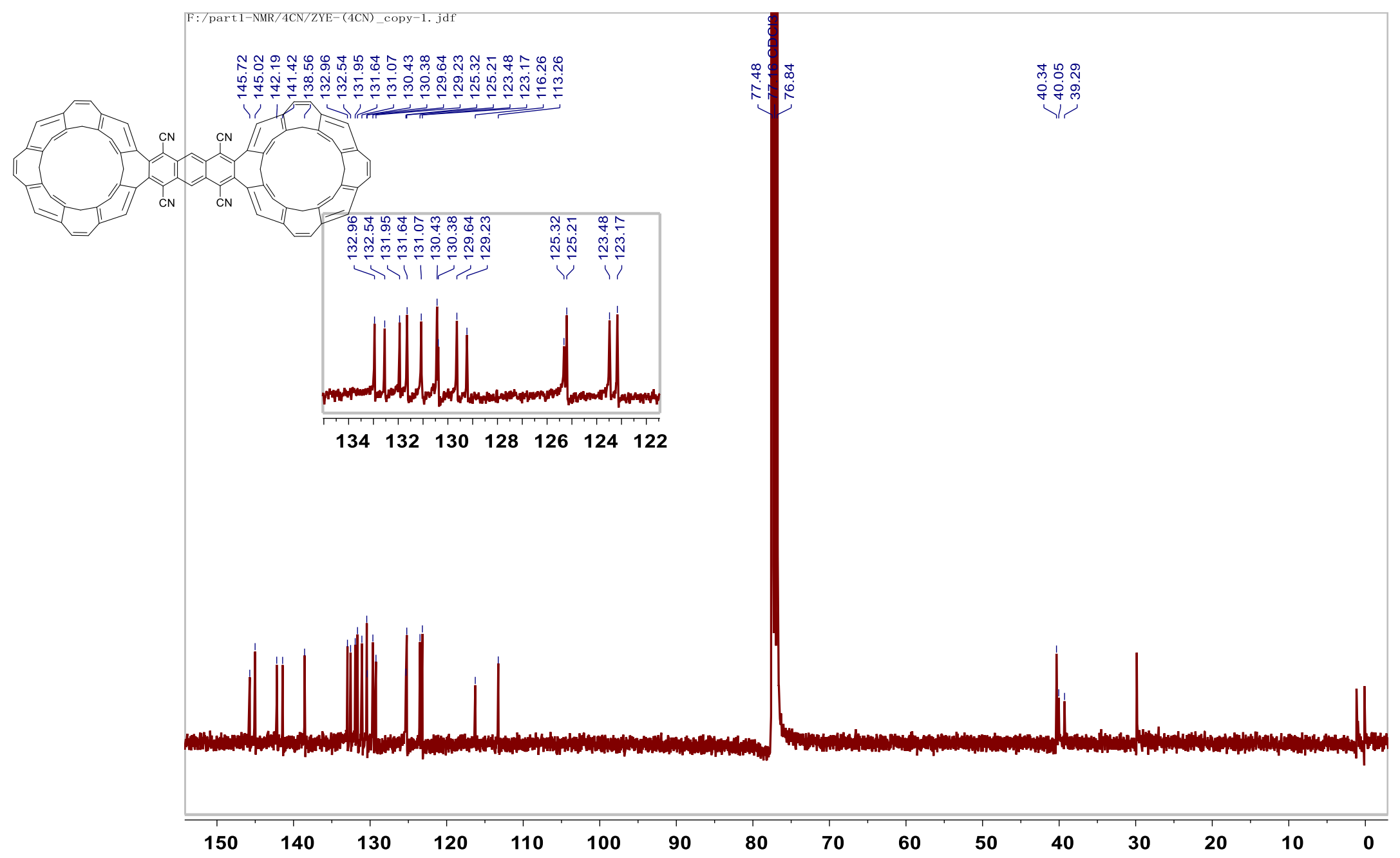


${ }^{1} \mathrm{H}$ NMR for 14 (C-shaped) (400 MHz)

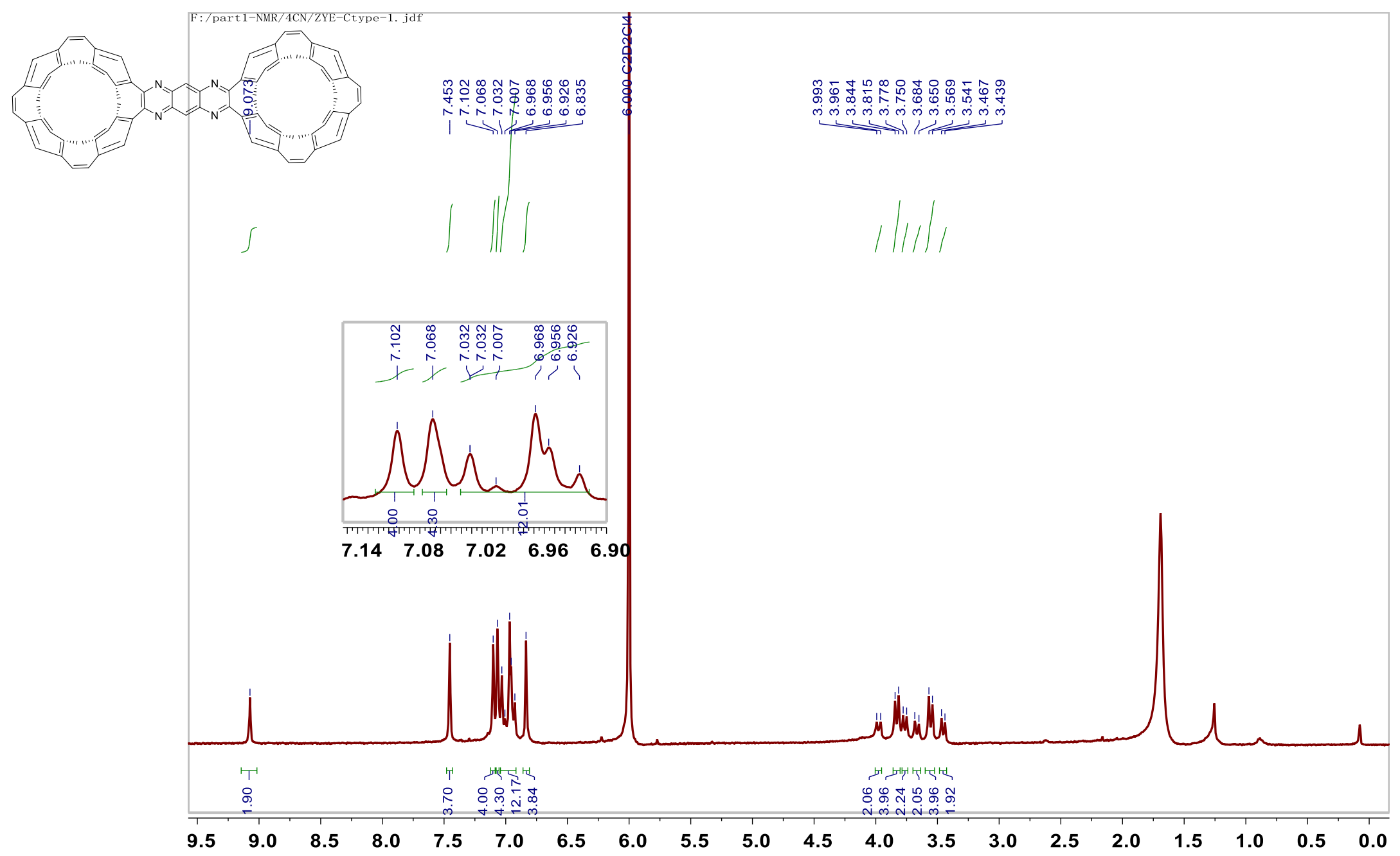


${ }^{1} \mathrm{H}$ NMR for 14 (S-shaped) (400 MHz)

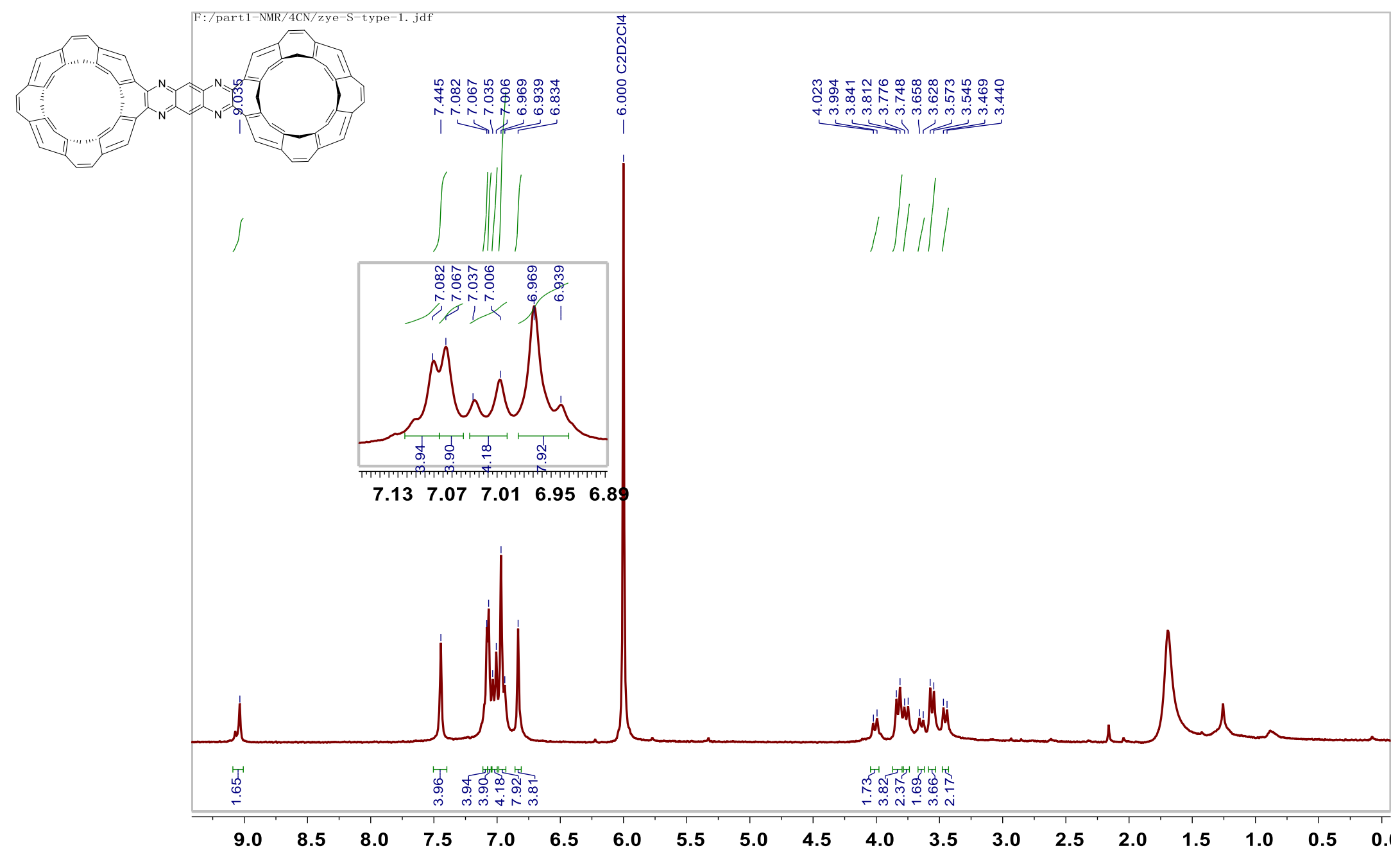


${ }^{1} \mathrm{H}$ NMR for 15 (400 MHz)

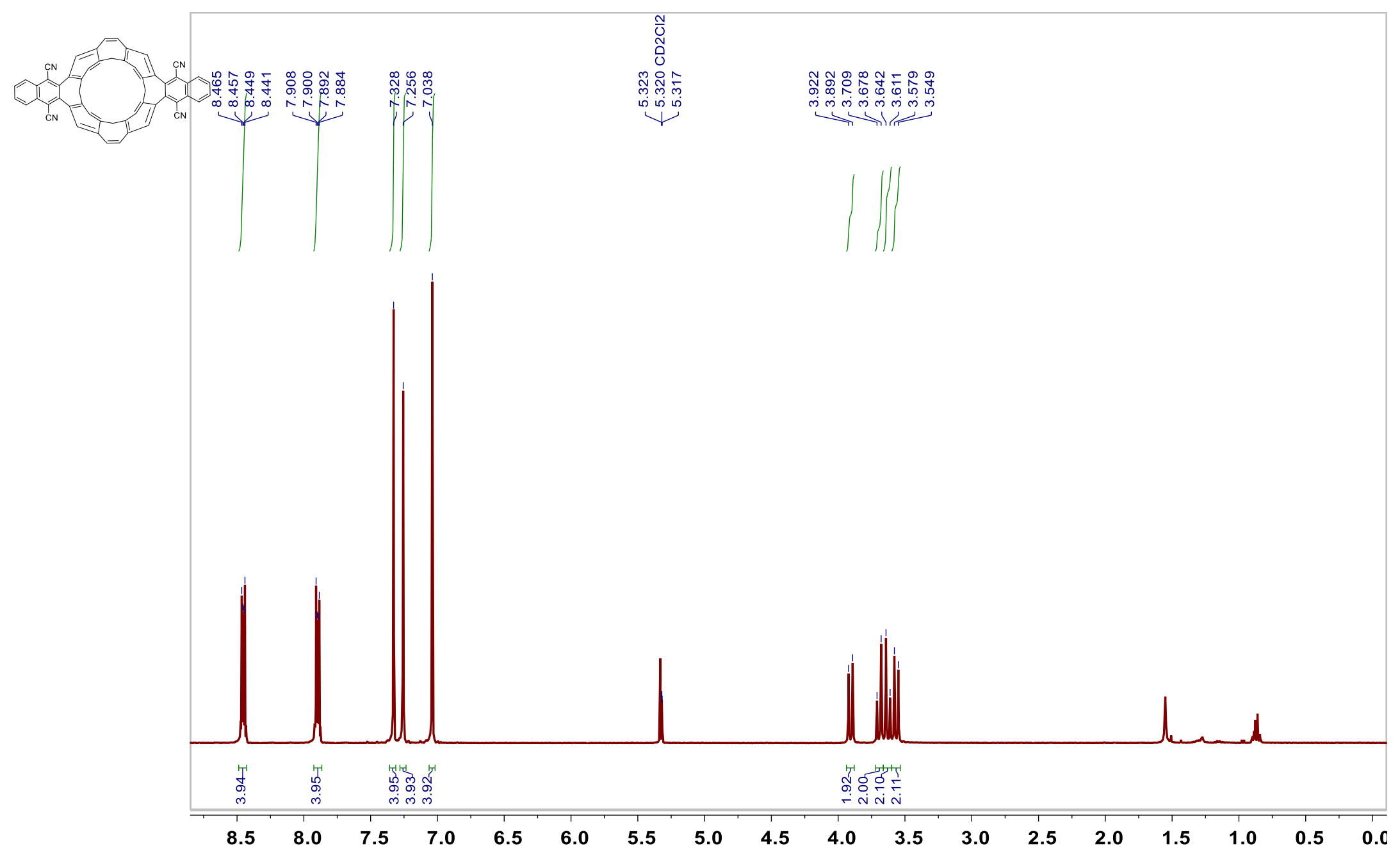


${ }^{13} \mathrm{C}$ NMR for 15 (100 MHz)

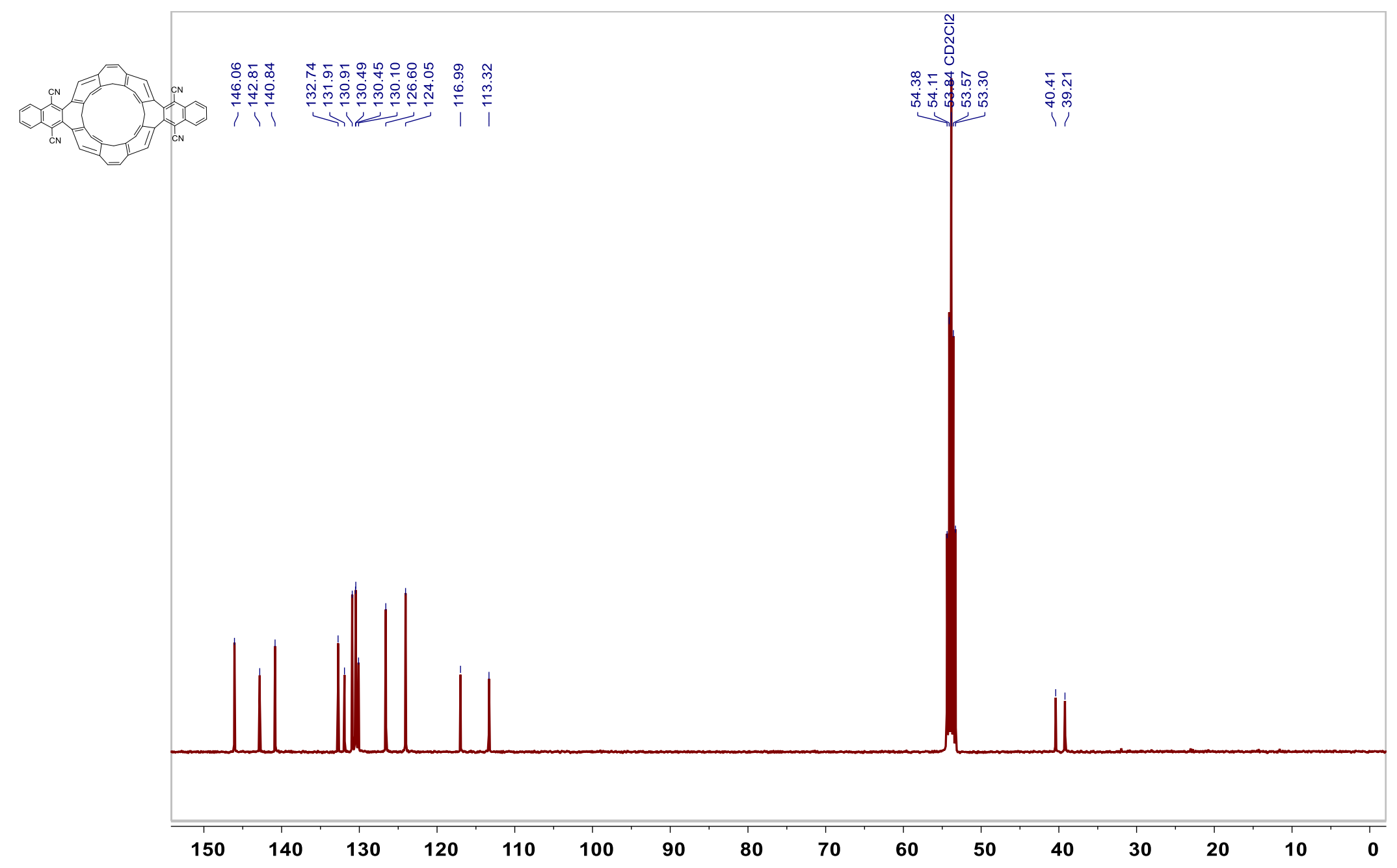


${ }^{1} \mathrm{H}$ NMR for 16 (400 MHz)

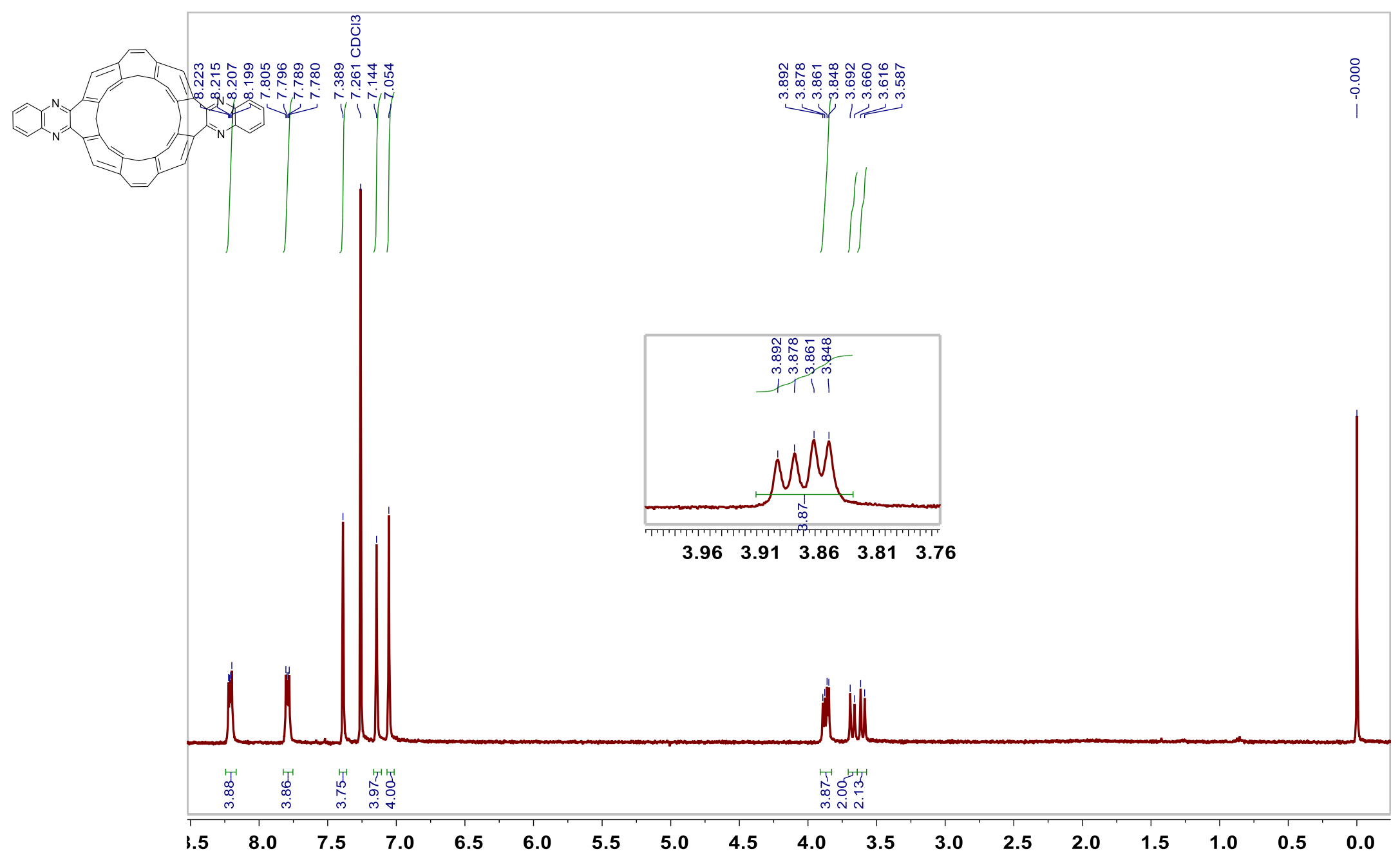


${ }^{13} \mathrm{C}$ NMR for $16(100 \mathrm{MHz})$

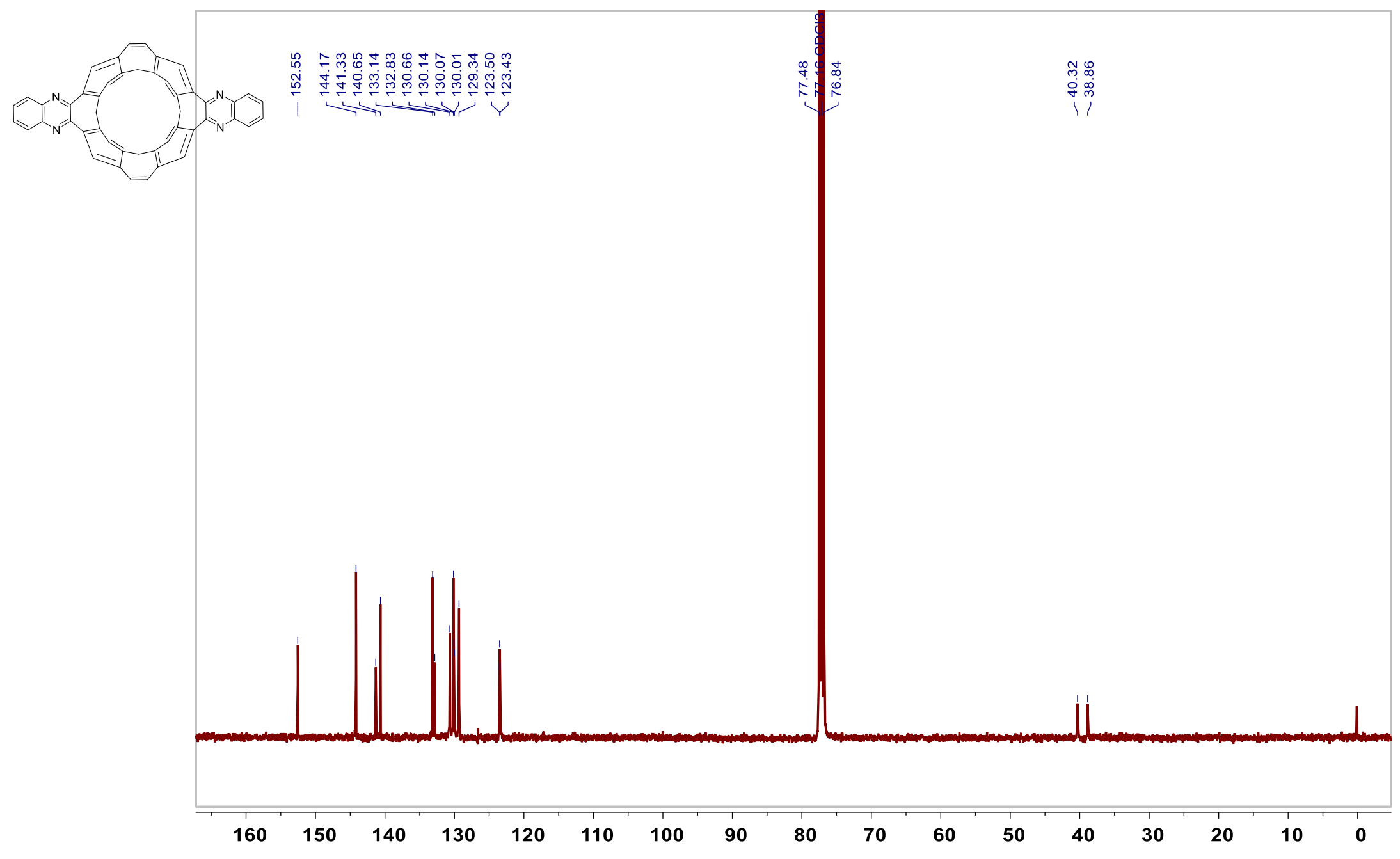

S45 


\section{${ }^{1} \mathrm{H}$ NMR for 17 (400 MHz)}

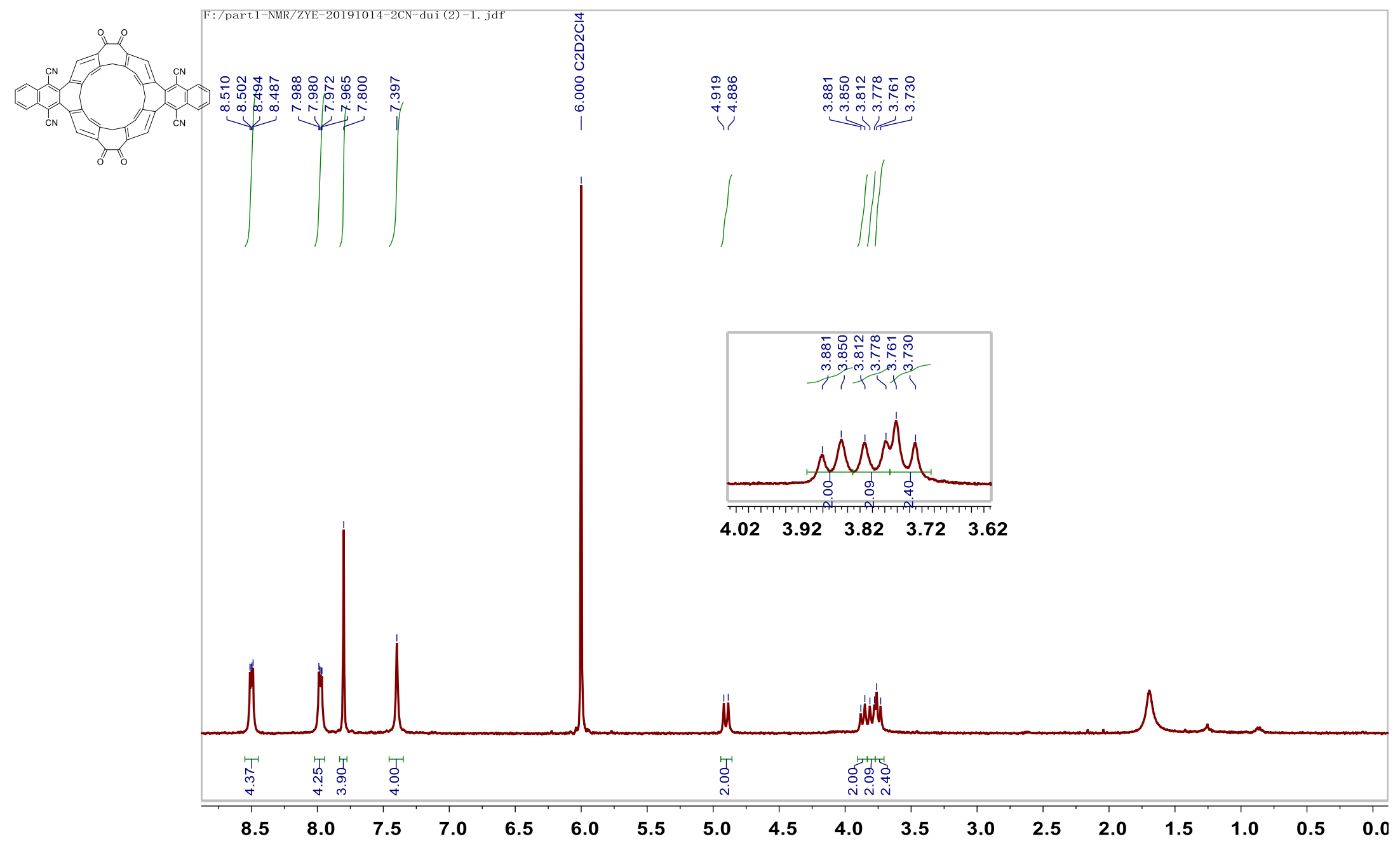


${ }^{13} \mathrm{C}$ NMR for $17(175 \mathrm{MHz})$

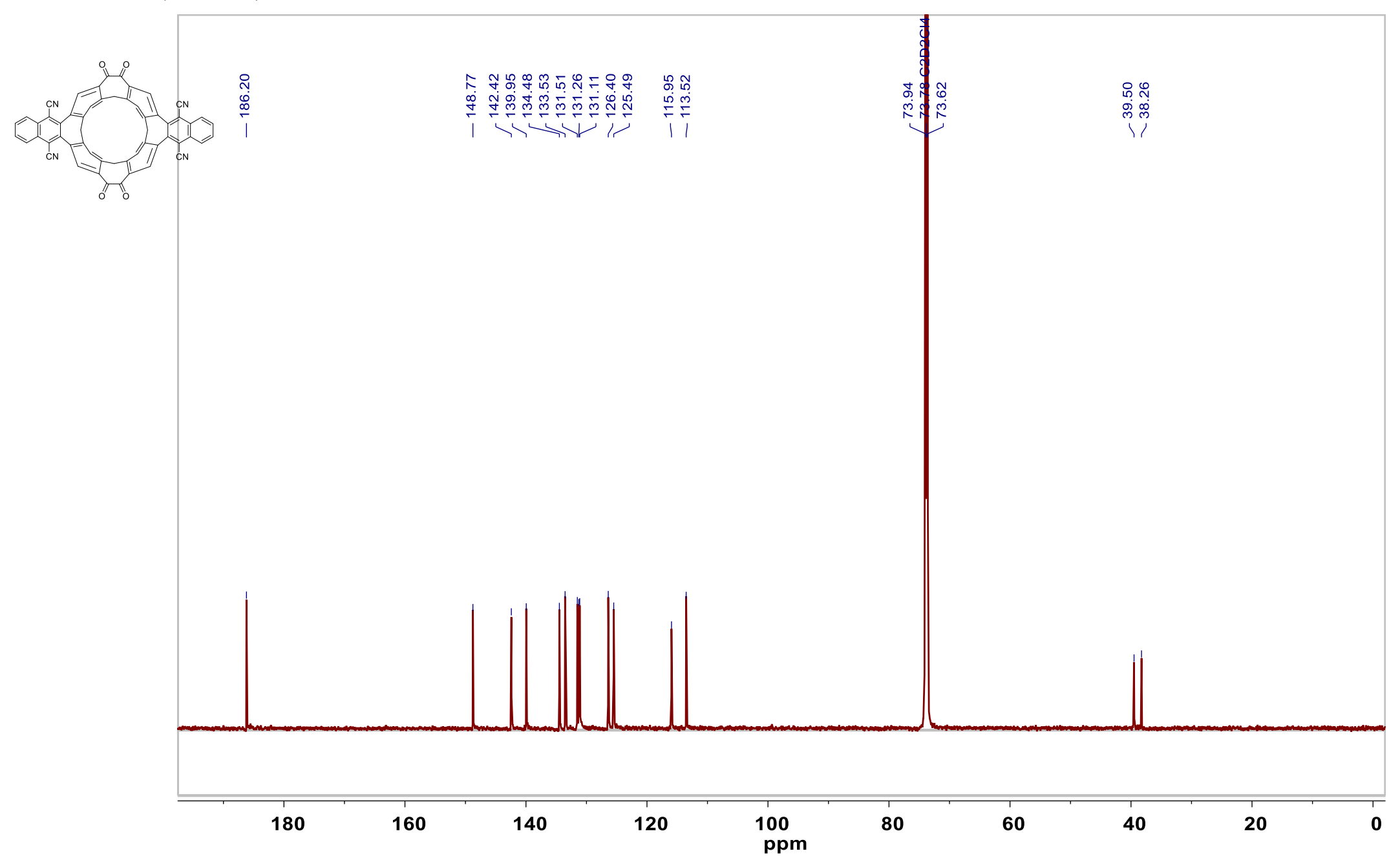

S47 
${ }^{1} \mathrm{H}$ NMR for 18 (400 MHz)

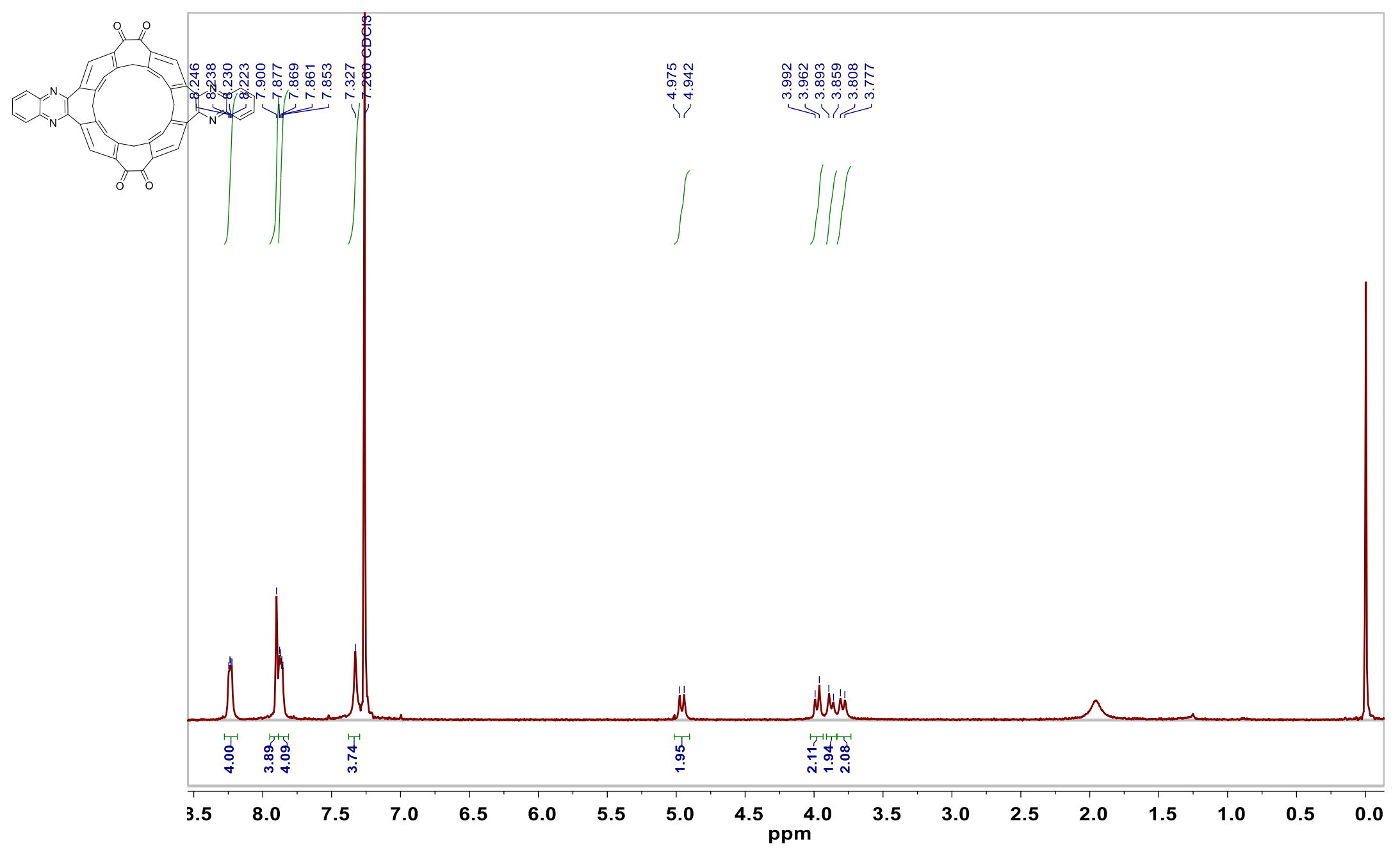


${ }^{13} \mathrm{C}$ NMR for $18(100 \mathrm{MHz})$

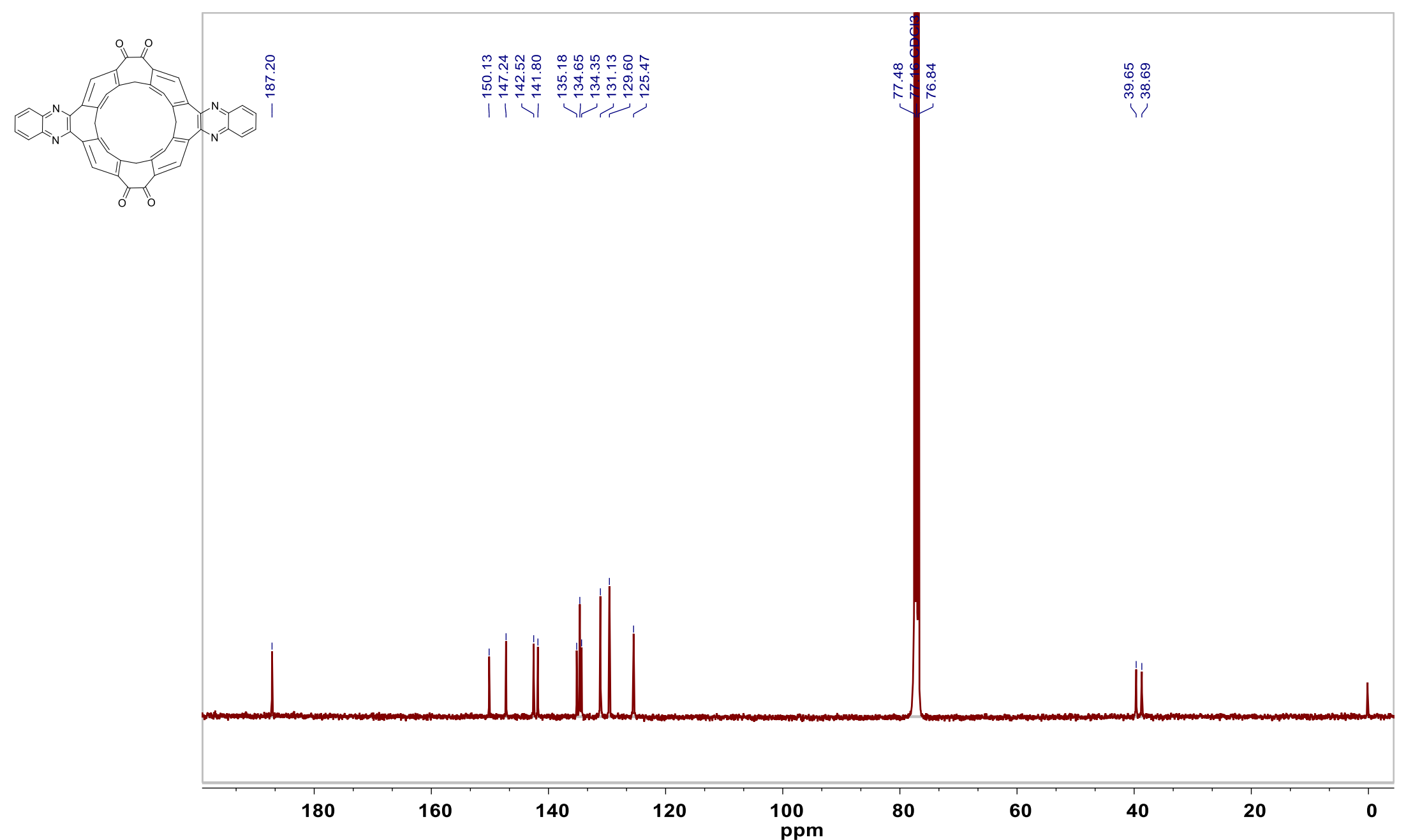


${ }^{1} \mathrm{H}$ NMR for 19 (400 MHz)

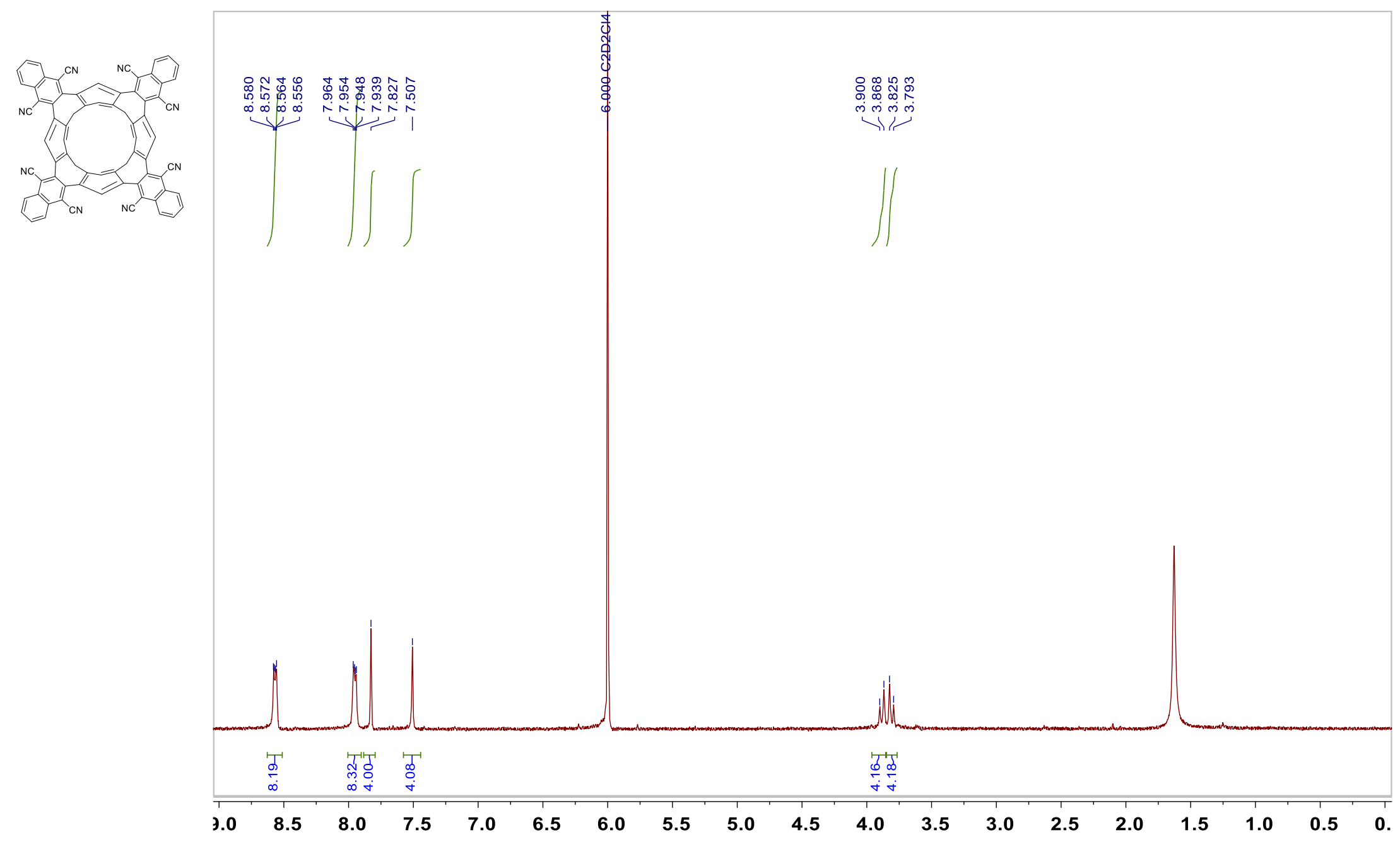


${ }^{13} \mathrm{C}$ NMR for 19 (100 MHz)

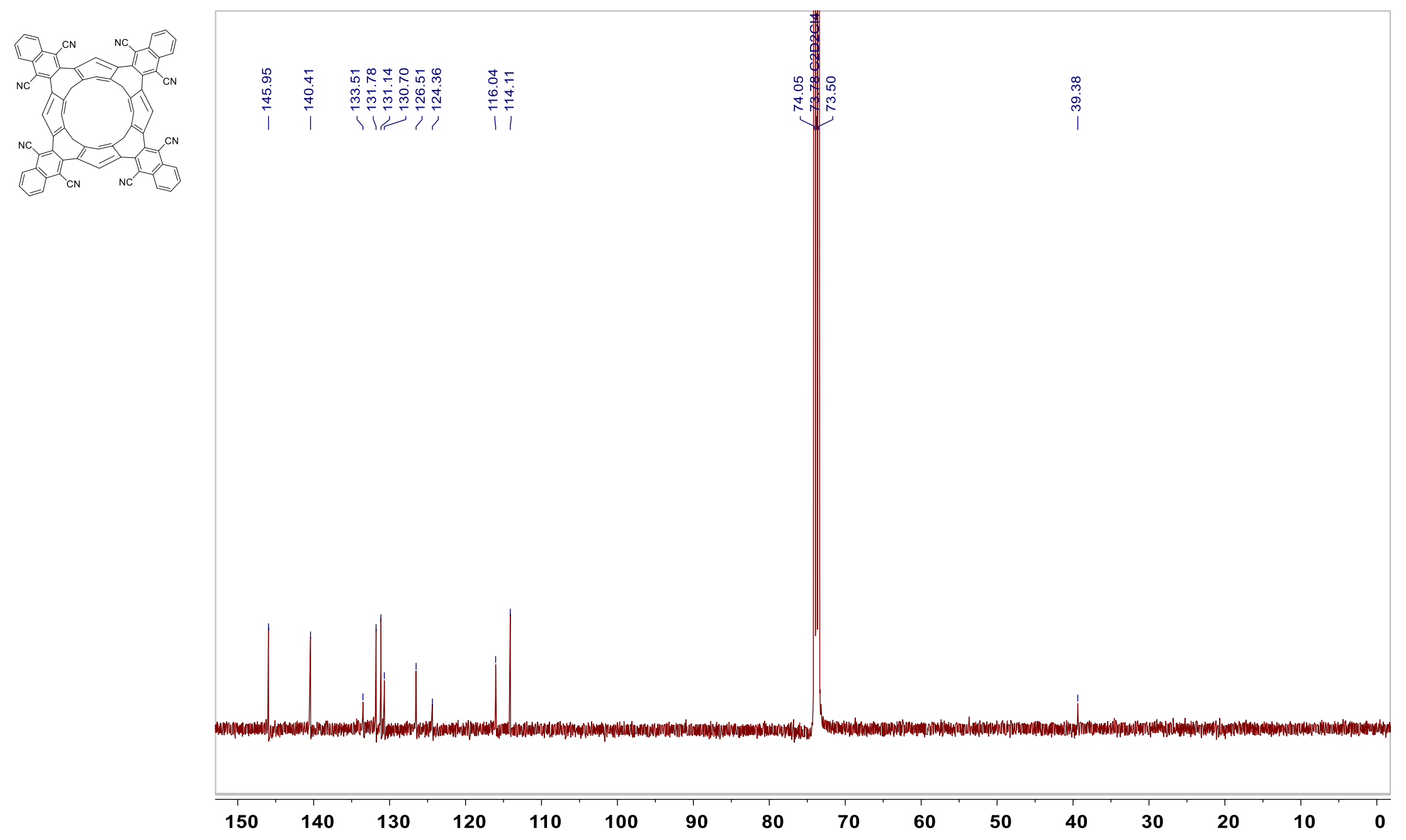


${ }^{1} \mathrm{H}$ NMR for 20 (400 MHz)

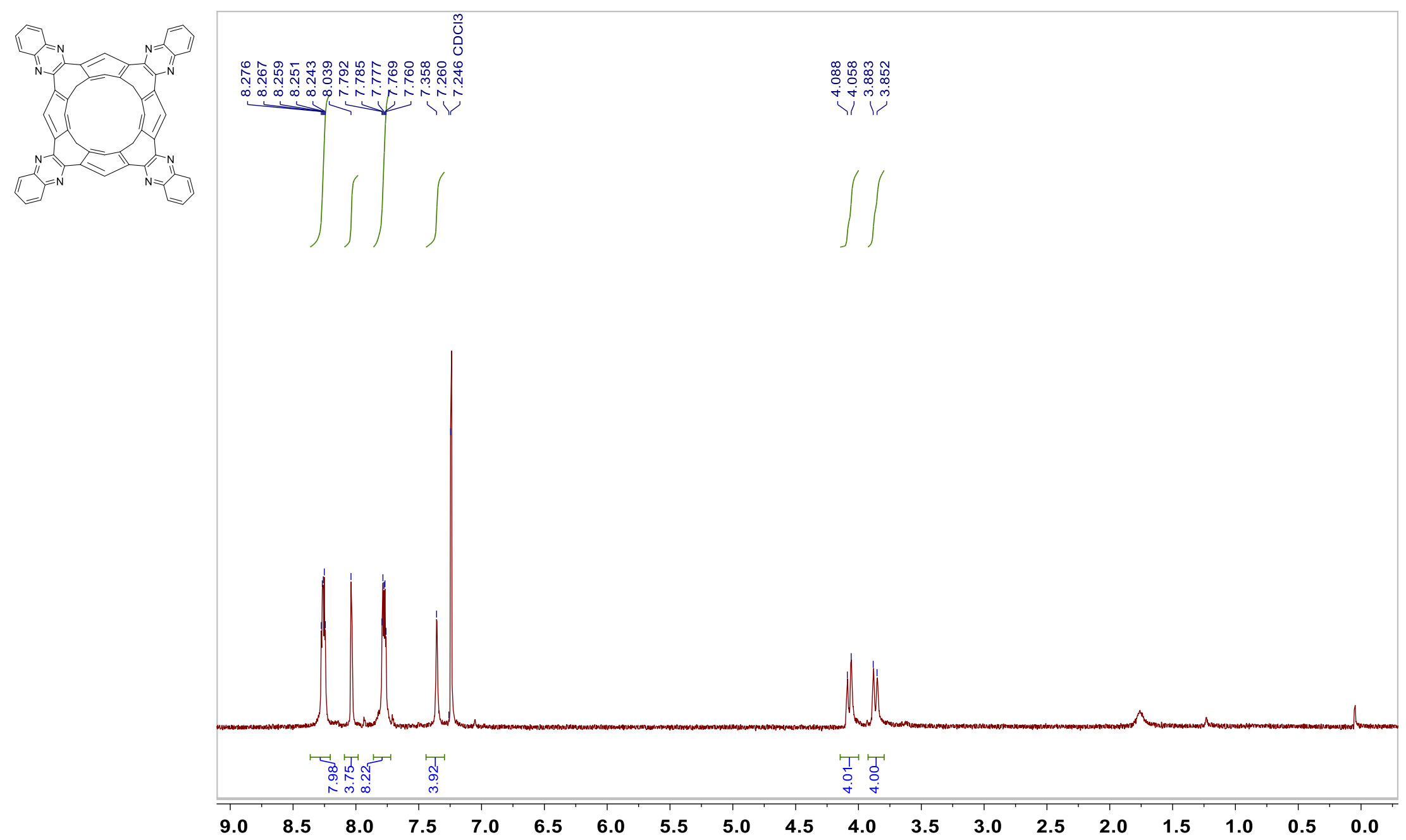


${ }^{13} \mathrm{C}$ NMR for 20 (100 MHz)

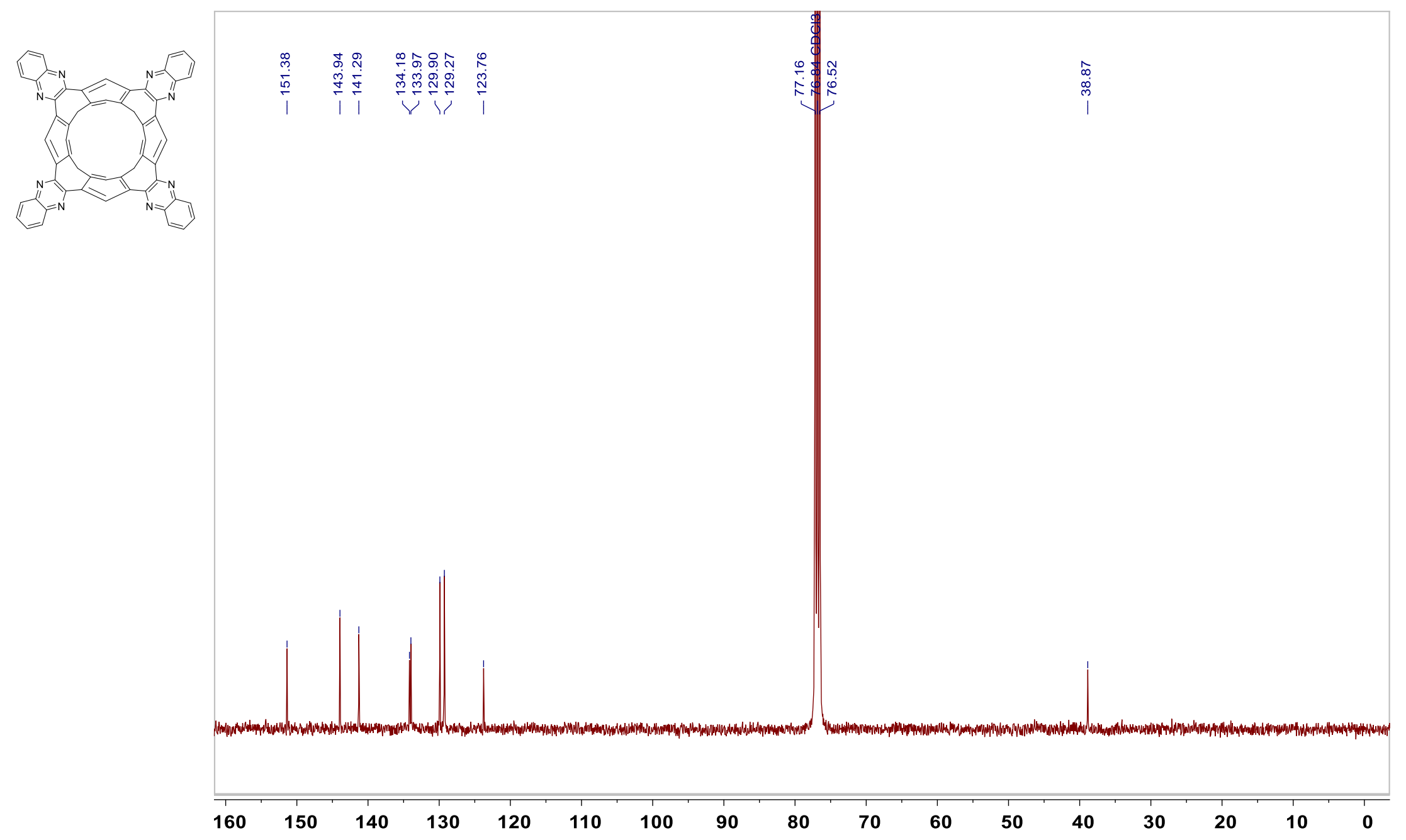

